Synthesis, antiviral activity, and mechanisms of purine nucleoside derivatives containing a sulfonamide moiety

Fangcheng He, ${ }^{\dagger}$ Jing Shi, ${ }^{\dagger}$ Yanju Wang, ${ }^{\dagger}$ Shaobo Wang, ${ }^{\dagger}$ JiXiang Chen,,${ }^{\dagger}$ Xiuhai Gan, ${ }^{\dagger}$ Baoan Song,${ }^{*}{ }^{\dagger}$ Deyu Hu $*, \uparrow$

$\uparrow$ State Key Laboratory Breeding Base of Green Pesticide and Agricultural Bioengineering, Key Laboratory of Green Pesticide and Agricultural Bioengineering, Ministry of Education, Guizhou University, Huaxi District, Guiyang 550025, China *Corresponding author (Tel.: 86-851-88292170; Fax: 86-851-88292170; E-mail:

dyhu@gzu.edu.cn; songbaoan22@yahoo.com) 


\section{General remarks}

The melting points of the products were determined on an XT-4 binocular microscope (Beijing Tech Instrument Co., China) and were not corrected. ${ }^{1} \mathrm{H}$ and ${ }^{13} \mathrm{C}$ nuclear magnetic resonance (NMR) (solvent DMSO- $d_{6}$ or $\mathrm{CDCl}_{3}$ ) spectra were performed on a JEOL-ECX 500 NMR spectrometer at room temperature using tetramethylsilane (TMS) as an internal standard. The following abbreviations were used to designate chemical shift multiplicities: $\mathrm{s}=$ singlet, $\mathrm{d}=$ doublet, $\mathrm{t}=$ triplet, $\mathrm{q}=$ quartet, $\mathrm{m}=$ multiplet. All first-order splitting patterns were assigned based on the appearance of the multiplet. Splitting patterns that could not be easily interpreted were designated as multiplet $(\mathrm{m})$. High resolution mass spectrometer (HRMS) data was conducted using a Thermo Scientific Q Exactive (Thermo, USA). Analytical thin-layer chromatography (TLC) was performed on silica gel $\mathrm{GF}_{254}$ (400 mesh). All of the reagents and reactants were purchased from commercial suppliers and of analytical reagent grade or chemically pure. All solvents were dried, deoxygenated, and redistilled before use.

General synthetic procedure for title compounds 1-25.
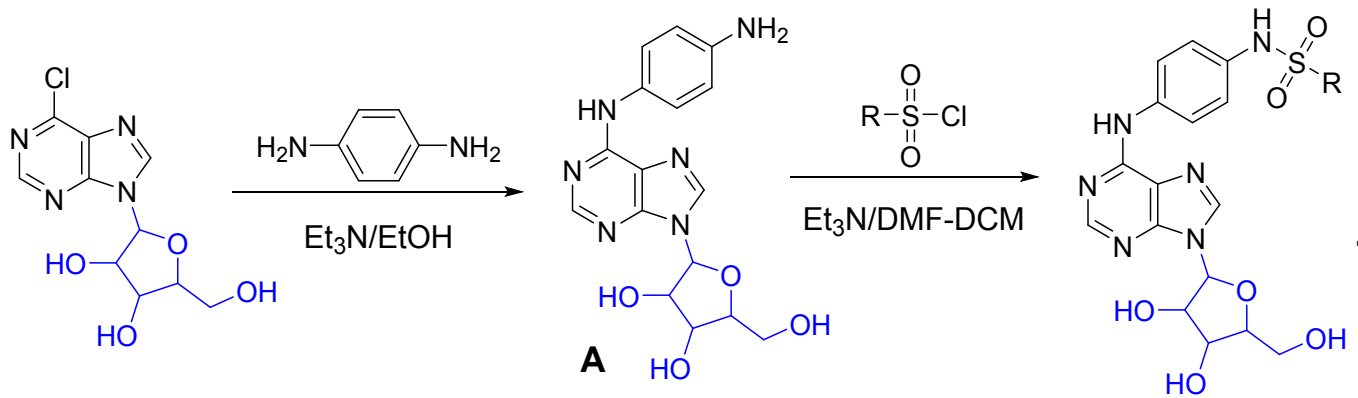

6-chloropurine nucleoside $(6.98 \mathrm{mmol})$ and $p$-phenylenediamine $(20.93 \mathrm{mmol})$ were used as reaction materials, and the mixture was heated under reflux for 12 hours under triethylamine $(7.67 \mathrm{mmol})$ as an acid-binding agent and ethanol as a solvent. Substitution reaction to synthesize intermediate A ((2-(6-((4-aminophenyl)amino)-9H -purin-9-yl)-5-(hydroxymethyl)tetrahydrofuran-3,4-diol). A reaction mixture of intermediate $\mathbf{A}(1.0 \mathrm{mmol})$ and triethylamine $(1.1 \mathrm{mmol})$ in $5 \mathrm{~mL} N$, $N$-dimethylformamide (DMF) and $15 \mathrm{~mL}$ dichloromethane (DCM) was stirred at $0{ }^{\circ} \mathrm{C}$ for 1 hour. Then, different sulfonyl chloride $(1.3 \mathrm{mmol})$ was added at $0{ }^{\circ} \mathrm{C}$ and stirred 
for 4 hours. The reaction mixture was washed with saturated saline, then extracted with $20 \mathrm{~mL}$ dichloromethane for three times and the organic layer was concentrated in a vacuum to obtain a colorless liquid, which was then washed with saturated brine and extracted with $20 \mathrm{~mL}$ ethyl acetate for three times. The ethyl acetate was removed and compounds 1-25 were obtained by recrystallization with dichloromethane. White or yellow solid with a yield of $62 \%$ to $80 \%$. The data of $\mathbf{1}$ to 25 was listed as follows.

\section{Characterization of products intermediate A.}

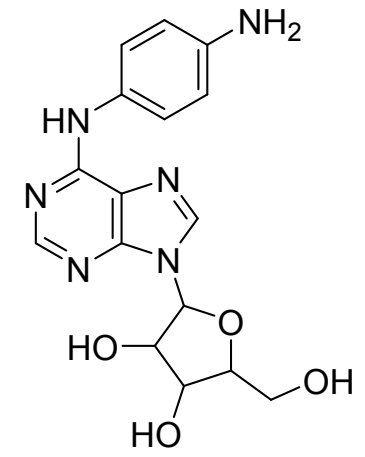

(2-(6-((4-aminophenyl)amino)-9H-purin-9-yl)-5-(hydroxym ethyl)tetrahydrofuran-3,4-diol) (intermediate A). Yield 98\%; gray solid; m.p. $178{ }^{\circ} \mathrm{C}-180{ }^{\circ} \mathrm{C} ;{ }^{1} \mathrm{H}$ NMR (500 MHz, DMSO- $\left.d_{6}\right) \delta 9.53(\mathrm{~s}, 1 \mathrm{H}$ Ph-NH), $8.49(\mathrm{~s}, 1 \mathrm{H}, \mathrm{Pu}-8$ 'H), 8.31 (s, 1H, Pu-2’H), 7.48 (d, $J=8.7 \mathrm{~Hz}, 2 \mathrm{H}, \mathrm{Ph}-\mathrm{H}), 6.60(\mathrm{~d}, J=8.6$ $\mathrm{Hz}, 2 \mathrm{H}, \mathrm{Ph}-\mathrm{H}), 5.97$ (d, $J=6.0 \mathrm{~Hz}, 1 \mathrm{H}$, ribose-2'CH), $5.52-5.40$ (m, 2H, ribose $\left.5^{\prime}-\mathrm{CH}_{2}-\right), 5.24\left(\mathrm{~d}, J=4.6 \mathrm{~Hz}, 1 \mathrm{H}\right.$, ribose 3'-CH), 4.93 (s, 2H, $\left.\mathrm{Ph}-\mathrm{NH}_{2}\right), 4.68$ (q, $J=$ $5.9 \mathrm{~Hz}, 1 \mathrm{H}$, ribose 4'-CH), $4.21(\mathrm{td}, J=4.7,2.8 \mathrm{~Hz}, 1 \mathrm{H}$, ribose 4'-OH), $4.03(\mathrm{~d}, J=$ $3.4 \mathrm{~Hz}, 1 \mathrm{H}$, ribose 5'-CH), $3.74\left(\mathrm{dt}, J=12.1,4.2 \mathrm{~Hz}, 1 \mathrm{H}\right.$, ribose $\left.3^{\prime}-\mathrm{OH}\right), 3.62(\mathrm{ddd}, J$ $=11.8,7.3,3.8 \mathrm{~Hz}, 1 \mathrm{H}$, ribose $\left.5^{\prime}-\mathrm{OH}\right) .{ }^{13} \mathrm{C}$ NMR (125 MHz, DMSO- $\left.d_{6}\right) \delta 152.94$, $152.49,149.19,145.30,140.47,128.54,123.65,120.35,114.06,88.34,86.29,73.92$, $71.03,62.04$.

\section{Characterization of products $1-25$.}

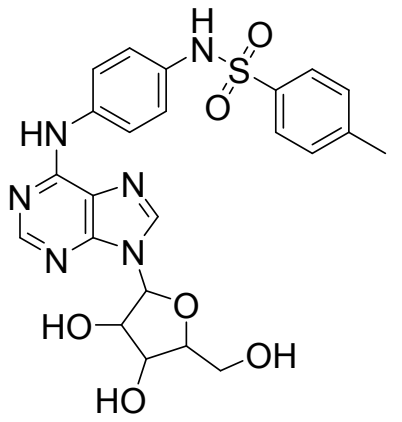

$N$-(4-((9-(3,4-dihydroxy-5-(hydroxymethyl)tetrahydrofur
an-2-yl)-9H-purin-6-yl)amino)phenyl)-4-methylbenzenes
ulfonamide (1): Gray solid; m.p. $184-186{ }^{\circ} \mathrm{C}$; Yield $62 \%$;
${ }^{1} \mathrm{H} \mathrm{NMR}\left(500 \mathrm{MHz}, \mathrm{DMSO}-d_{6}\right) \delta 10.15\left(\mathrm{~s}, 1 \mathrm{H},-\mathrm{SO}_{2}-\mathrm{NH}-\right)$, 
9.89 (s, 1H, Pu-8’H), 8.55 (s, 1H, Ph-NH), 8.37 (s, 1H, Pu-2’H), 7.79 (d, J = 8.7 Hz, 2H, Ph-H), 7.68 (d, $J=8.0 \mathrm{~Hz}, 2 \mathrm{H}, \mathrm{Ph}-\mathrm{H}), 7.36(\mathrm{~d}, J=7.8 \mathrm{~Hz}, 2 \mathrm{H}, \mathrm{Ph}-\mathrm{H}), 7.08$ (d, $J=$ $8.5 \mathrm{~Hz}, 2 \mathrm{H}, \mathrm{Ph}-\mathrm{H}), 5.98(\mathrm{~d}, J=5.9 \mathrm{~Hz}, 1 \mathrm{H}$, ribose 2'-CH), $5.56(\mathrm{~d}, J=5.8 \mathrm{~Hz}, 1 \mathrm{H}$, ribose $\left.3^{\prime}-\mathrm{CH}\right), 5.36-5.29\left(\mathrm{~m}, 2 \mathrm{H}\right.$, ribose $\left.5^{\prime}-\mathrm{CH}_{2}-\right), 4.65(\mathrm{~d}, J=5.9 \mathrm{~Hz}, 1 \mathrm{H}$, ribose 5'-CH), $4.22(\mathrm{~d}, J=5.0 \mathrm{~Hz}, 1 \mathrm{H}$, ribose 3'-OH), $4.02(\mathrm{~d}, J=5.0 \mathrm{~Hz}, 1 \mathrm{H}$, ribose 4'-CH), $3.75-3.67(\mathrm{~m}, 1 \mathrm{H}$, ribose 4'-OH), 3.60 (dd, $J=12.1,6.4 \mathrm{~Hz}, 1 \mathrm{H}$, ribose 5'-OH), 2.35 (s, 3H, Ph- $\left.\mathrm{CH}_{3}\right) .{ }^{13} \mathrm{C}$ NMR (125 MHz, DMSO-d $) \delta$ 152.43, 149.82, $143.58,141.17,137.33,136.59,133.02,130.15,127.32,122.13,121.67,120.72$, 88.34, 86.39, 74.22, 71.09, 62.06, 21.50. HRMS (ESI) calcd for $\mathrm{C}_{23} \mathrm{H}_{25} \mathrm{O}_{6} \mathrm{~N}_{6} \mathrm{~S}$ $\left([\mathrm{M}+\mathrm{H}]^{+}\right)$, 513.14780, found. 513.15411.

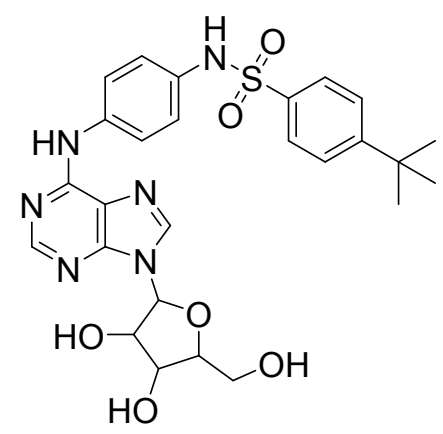

4-(tert-butyl)- $N$-(4-((9-(3,4-dihydroxy-5-(hydroxyme thyl)tetrahydrofuran-2-yl)-9H-purin-6-yl)amino)phe nyl)benzenesulfonamide. (2): Yellow solid; m.p. 174-176 ${ }^{\circ} \mathrm{C}$; Yield 78\%; ${ }^{1} \mathrm{H}$ NMR $(500 \mathrm{MHz}$, DMSO- $\left.d_{6}\right) \delta 10.21\left(\mathrm{~s}, 1 \mathrm{H},-\mathrm{SO}_{2}-\mathrm{NH}-\right), 9.86(\mathrm{~s}, 1 \mathrm{H}$, Pu-8’H), 8.50 (s, 1H, Ph-NH), 8.30 (s, 1H, Pu-2’H), 7.73 (d, $J=8.6 \mathrm{~Hz}, 2 \mathrm{H}, \mathrm{Ph}-\mathrm{H}), 7.68$ (d, $J=8.3 \mathrm{~Hz}, 2 \mathrm{H}, \mathrm{Ph}-\mathrm{H}), 7.54$ (d, $J=8.3 \mathrm{~Hz}, 2 \mathrm{H}$, Ph-H), 7.05 (d, $J=8.8 \mathrm{~Hz}, 2 \mathrm{H}, \mathrm{Ph}-\mathrm{H}), 5.90$ (d, $J=6.0 \mathrm{~Hz}, 1 \mathrm{H}$, ribose 2'-CH), 5.53 (d, $J=6.1 \mathrm{~Hz}, 1 \mathrm{H}$, ribose $\left.3^{\prime}-\mathrm{CH}\right), 5.32-5.26\left(\mathrm{~m}, 2 \mathrm{H}\right.$, ribose $\left.5^{\prime}-\mathrm{CH}_{2^{-}}\right), 4.58(\mathrm{q}, J=5.7$ $\mathrm{Hz}, 1 \mathrm{H}$, ribose 5'-CH), 4.13 (q, $J=4.4 \mathrm{~Hz}, 1 \mathrm{H}$, ribose 3'-OH), 3.94 (d, $J=3.5 \mathrm{~Hz}, 1 \mathrm{H}$, ribose 4'-CH), $3.66-3.62(\mathrm{~m}, 1 \mathrm{H}$, ribose 4'-OH), 3.52 (ddd, $J=11.6,6.7,3.8 \mathrm{~Hz}, 1 \mathrm{H}$, ribose 5'-OH), $1.22\left(\mathrm{~s}, 9 \mathrm{H}, \mathrm{Ph}-3\left(\mathrm{CH}_{3}\right)_{3}\right) .{ }^{13} \mathrm{C}$ NMR (125 MHz, DMSO-d $\left.d_{6}\right) \delta 156.22$, $152.45,149.84,141.18,137.62,136.47,133.14,127.15,126.60,122.18,121.56$, 121.26, 120.74, 88.34, 86.39, 74.23, 71.09, 62.06, 35.38, 31.28. HRMS (ESI): calcd for $\mathrm{C}_{26} \mathrm{H}_{31} \mathrm{O}_{6} \mathrm{~N}_{6} \mathrm{~S}\left([\mathrm{M}+\mathrm{H}]^{+}\right)$, 555.19475; found, 555.20105. 


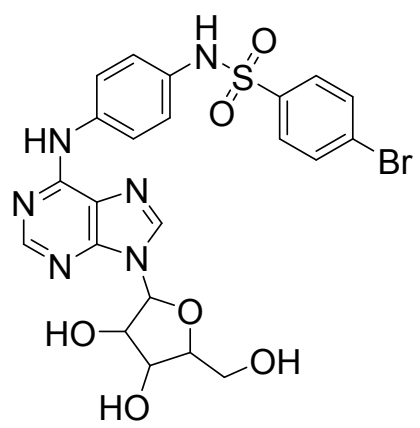

4-bromo-N-(4-((9-(3,4-dihydroxy-5-(hydroxymethyl)tet rahydrofuran-2-yl)-9H-purin-6-yl)amino)phenyl)benze nesulfonamide (3). Yellow solid. m.p. $158-160{ }^{\circ} \mathrm{C}$; Yield $76 \%$; ${ }^{1} \mathrm{H}$ NMR $\left(500 \mathrm{MHz}, \mathrm{DMSO}-d_{6}\right) \delta 10.26(\mathrm{~s}, 1 \mathrm{H}$, -SO $\left.\mathrm{SO}_{2} \mathrm{NH}-\right), 9.96$ (s, 1H, Pu-8'H), 8.58 (s, 1H, Ph-NH), $8.42\left(\mathrm{~s}, 1 \mathrm{H}, \mathrm{Pu}-2^{\prime} \mathrm{H}\right), 7.85(\mathrm{dd}, J=16.7,8.4 \mathrm{~Hz}, 4 \mathrm{H}$, Ph-H), 7.72 (d, $J=8.0$ Hz, 2H, Ph-H), 7.09 (d, $J=8.5$ Hz, 2H, Ph-H), 6.00 (d, $J=6.0$ $\mathrm{Hz}, 1 \mathrm{H}$, ribose 2' $-\mathrm{CH}), 5.54(\mathrm{~d}, J=6.3 \mathrm{~Hz}, 1 \mathrm{H}$, ribose 3'-CH), $5.35-5.26(\mathrm{~m}, 2 \mathrm{H}$, ribose 5'- $\mathrm{CH}_{2}$ ), $4.68(\mathrm{q}, J=5.8 \mathrm{~Hz}, 1 \mathrm{H}$, ribose 5'-CH), $4.23(\mathrm{~d}, J=4.1 \mathrm{~Hz}, 1 \mathrm{H}$, ribose 3'-OH), $4.04(\mathrm{~d}, J=3.5 \mathrm{~Hz}, 1 \mathrm{H}$, ribose 4'-CH), $3.74(\mathrm{dt}, J=12.5,4.4 \mathrm{~Hz}, 1 \mathrm{H}$, ribose 4'-OH), 3.63 (ddd, $J=11.7,6.6,3.8 \mathrm{~Hz}, 1 \mathrm{H}$ ribose $\left.5^{\prime}-\mathrm{OH}\right) .{ }^{13} \mathrm{C}$ NMR $(125$ MHz, DMSO- $\left.d_{6}\right) \delta 152.42,149.84,141.22,139.39,137.09,134.44,132.86,132.35$, $129.28,127.19,122.54,122.12,120.80,88.36,86.39,74.15,71.08,62.08$. HRMS (ESI): calcd for $\mathrm{C}_{22} \mathrm{H}_{22} \mathrm{O}_{6} \mathrm{~N}_{6} \mathrm{BrS}\left([\mathrm{M}+\mathrm{H}]^{+}\right)$, 577.04267; found, 577.04858.

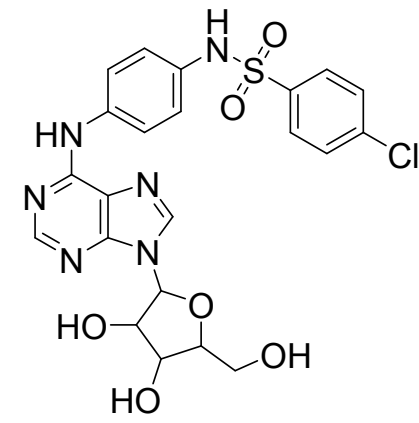

4-chloro- $N$-(4-((9-(3,4-dihydroxy-5-(hydroxymethyl)tetrahy drofuran-2-yl)-9H-purin-6-yl)amino)phenyl)benzenesulfon amide (4). White solid; m.p. $78-79{ }^{\circ} \mathrm{C}$; Yield 76\%; ${ }^{1} \mathrm{H}$ NMR $\left(500 \mathrm{MHz}, \mathrm{DMSO}-d_{6}\right) \delta 10.27\left(\mathrm{~s}, 1 \mathrm{H},-\mathrm{SO}_{2}-\mathrm{NH}-\right), 9.92(\mathrm{~s}, 1 \mathrm{H}$, Pu-8’H), 8.51 (s, 1H, Ph-NH), 8.32 (s, 1H, Pu-2'H), 7.77 (d, J $=8.9 \mathrm{~Hz}, 2 \mathrm{H}, \mathrm{Ph}-\mathrm{H}), 7.72(\mathrm{~d}, J=8.6 \mathrm{~Hz}, 2 \mathrm{H}, \mathrm{Ph}-\mathrm{H}), 7.60(\mathrm{~d}, J$ $=8.6 \mathrm{~Hz}, 2 \mathrm{H}, \mathrm{Ph}-\mathrm{H}), 7.01(\mathrm{~d}, J=8.9 \mathrm{~Hz}, 2 \mathrm{H}, \mathrm{Ph}-\mathrm{H}), 5.91(\mathrm{~d}, J=6.1 \mathrm{~Hz}, 1 \mathrm{H}$, ribose 2'-CH), $5.52(\mathrm{~d}, J=6.1 \mathrm{~Hz}, 1 \mathrm{H}$, ribose 3'- $\mathrm{CH}), 5.32-5.25\left(\mathrm{~m}, 2 \mathrm{H}\right.$, ribose 5'- $\left.\mathrm{CH}_{2^{-}}\right), 4.59$ (q, $J=5.8 \mathrm{~Hz}, 1 \mathrm{H}$, ribose 5'-CH), $4.13\left(\mathrm{q}, J=4.3 \mathrm{~Hz}, 1 \mathrm{H}\right.$, ribose $\left.3^{\prime}-\mathrm{OH}\right), 3.94(\mathrm{q}, J=3.6$ $\mathrm{Hz}, 1 \mathrm{H}$, ribose 4'-CH), 3.64 (dt, $J=12.1,4.4 \mathrm{~Hz}, 1 \mathrm{H}$, ribose 4'-OH), 3.53 (ddd, $J=11.5$, $6.8,3.8 \mathrm{~Hz}, 1 \mathrm{H}$, ribose $\left.5^{\prime}-\mathrm{OH}\right) \cdot{ }^{13} \mathrm{C}$ NMR $\left(125 \mathrm{MHz}, \mathrm{DMSO}-d_{6}\right) \delta 152.44,149.88$, $141.22,139.04,138.17,137.08,132.45,129.91,129.24,122.26,120.79,88.35,86.39$, 74.21, 71.09, 62.07. HRMS (ESI): calcd for $\mathrm{C}_{22} \mathrm{H}_{22} \mathrm{O}_{6} \mathrm{~N}_{6} \mathrm{ClS}\left([\mathrm{M}+\mathrm{H}]^{+}\right), 533.09318$; found, 533.09918. 


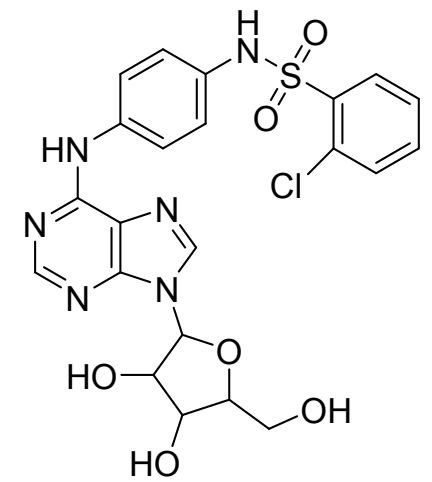

2-chloro- $N$-(4-((9-(3,4-dihydroxy-5-(hydroxymethyl)tetrahy drofuran-2-yl)-9H-purin-6-yl)amino)phenyl)benzenesulfon amide (5) White solid; m.p. $152-154{ }^{\circ} \mathrm{C}$; Yield 75\%; ${ }^{1} \mathrm{H}$ NMR $\left(500 \mathrm{MHz}, \mathrm{DMSO}-d_{6}\right) \delta 10.44\left(\mathrm{~s}, 1 \mathrm{H},-\mathrm{SO}_{2}-\mathrm{NH}-\right), 9.88(\mathrm{~s}, 1 \mathrm{H}$, Pu-8'H), 8.49 (s, 1H, Ph-NH), 8.30 (s, 1H, Pu-2'H), 7.98 (dd, $J=8.0,1.6 \mathrm{~Hz}, 1 \mathrm{H}, \mathrm{Ph}-\mathrm{H}), 7.72(\mathrm{~d}, J=9.0 \mathrm{~Hz}, 2 \mathrm{H}, \mathrm{Ph}-\mathrm{H})$, $7.62-7.58$ (m, 2H, Ph-H), 7.47 (ddd, $J=8.3,7.2,1.6 \mathrm{~Hz}, 1 \mathrm{H}, \mathrm{Ph}-\mathrm{H}), 7.03$ (d, $J=9.0$ $\mathrm{Hz}, 2 \mathrm{H}, \mathrm{Ph}-\mathrm{H}), 5.90$ (d, $J=6.1 \mathrm{~Hz}, 1 \mathrm{H}$, ribose 2'-CH), 5.49 (d, $J=6.1 \mathrm{~Hz}, 1 \mathrm{H}$, ribose 3'-CH), $5.30-5.22\left(\mathrm{~m}, 2 \mathrm{H}\right.$, ribose 5' $\left.-\mathrm{CH}_{2}-\right), 4.58(\mathrm{q}, J=5.8 \mathrm{~Hz}, 1 \mathrm{H}$, ribose 5'-CH), 4.12 (td, $J=4.8,3.1 \mathrm{~Hz}, 1 \mathrm{H}$, ribose 3'-OH), 3.93 (q, $J=3.6 \mathrm{~Hz}, 1 \mathrm{H}$, ribose 4'-CH), 3.64 (dt, $J$ $=12.1,4.4 \mathrm{~Hz}, 1 \mathrm{H}$, ribose $\left.4^{\prime}-\mathrm{OH}\right), 3.55-3.50\left(\mathrm{~m}, 1 \mathrm{H}\right.$, ribose $\left.5^{\prime}-\mathrm{OH}\right) .{ }^{13} \mathrm{C}$ NMR $(125$ MHz, DMSO- $\left.d_{6}\right) \delta 152.48,151.44,149.80,141.23,137.12,136.61,135.14,134.37$, $133.18,132.13,131.27,128.29,122.25,121.02,120.73,88.31,86.39,74.14,71.08$, 62.06. HRMS (ESI): calcd for $\mathrm{C}_{22} \mathrm{H}_{22} \mathrm{O}_{6} \mathrm{~N}_{6} \mathrm{ClS}\left([\mathrm{M}+\mathrm{H}]^{+}\right)$, 533.09318; found, 533.09937.

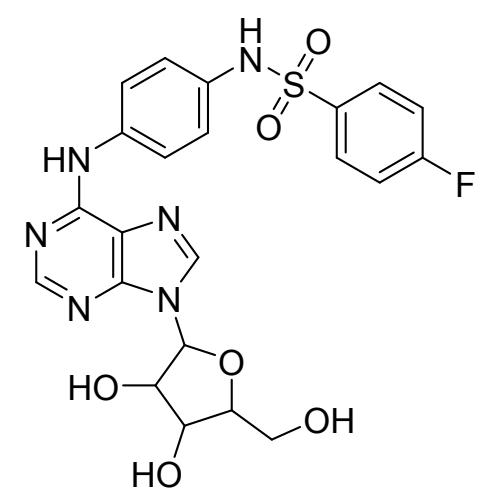

N-(4-((9-(3,4-dihydroxy-5-(hydroxymethyl)tetrahydr ofuran-2-yl)-9H-purin-6-yl)amino)phenyl)-4-fluorob enzenesulfonamide (6). White solid; m.p. $172-174{ }^{\circ} \mathrm{C}$; Yield 78\%; ${ }^{1} \mathrm{H}$ NMR $\left(500 \mathrm{MHz}, \mathrm{DMSO}-d_{6}\right) \delta 10.17$ (s, $\left.1 \mathrm{H},-\mathrm{SO}_{2}-\mathrm{NH}-\right), 9.91\left(\mathrm{~s}, 1 \mathrm{H}, \mathrm{Pu}-8^{\prime} \mathrm{H}\right), 8.51(\mathrm{~s}, 1 \mathrm{H}$, Ph-NH), 8.32 (s, 1H, Pu-2'H), 7.78 - 7.76 (m, 4H, Ph-H), 7.36 (d, $J=2.5 \mathrm{~Hz}, 2 \mathrm{H}, \mathrm{Ph}-\mathrm{H}), 7.00(\mathrm{~d}, J=3.9$ $\mathrm{Hz}, 2 \mathrm{H}, \mathrm{Ph}-\mathrm{H}), 5.91$ (d, $J=6.1 \mathrm{~Hz}, 1 \mathrm{H}$, ribose2'-CH), 5.52 (d, $J=6.1 \mathrm{~Hz}, 1 \mathrm{H}$, ribose3'-CH), $5.33-5.24\left(\mathrm{~m}, 2 \mathrm{H}\right.$, ribose 5' $\left.-\mathrm{CH}_{2}-\right), 4.59(\mathrm{~d}, J=5.5 \mathrm{~Hz}, 1 \mathrm{H}$, ribose5'-CH), $4.13(\mathrm{~d}, J=4.1 \mathrm{~Hz}, 1 \mathrm{H}$, ribose3'-OH), $3.94(\mathrm{~d}, J=3.5 \mathrm{~Hz}, 1 \mathrm{H}$, ribose4'-CH), $3.68-3.62(\mathrm{~m}, 1 \mathrm{H}$, ribose4'-OH), 3.53 (ddd, $J=11.4,6.6,3.8 \mathrm{~Hz}, 1 \mathrm{H}$ ribose5'-OH). ${ }^{13} \mathrm{C}$ NMR (125 MHz, DMSO- $\left.d_{6}\right) \delta 165.76,163.76,152.46,152.26$, $149.84,141.24,136.95,136.44,132.60,132.14,130.31,122.08,120.74,117.02$, 116.84, 88.29, 86.39, 74.19, 71.08, 62.04. HRMS (ESI): calcd for $\mathrm{C}_{22} \mathrm{H}_{22} \mathrm{O}_{6} \mathrm{~N}_{6} \mathrm{FS}$ $\left([\mathrm{M}+\mathrm{H}]^{+}\right), 517.12273$; found, 517.12872. 


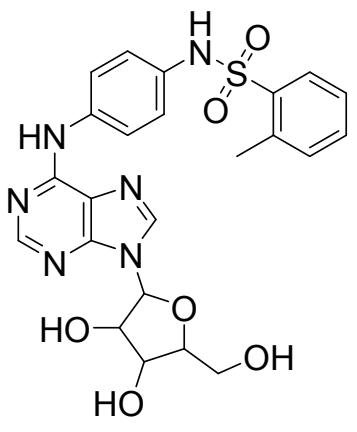

N-(4-((9-(3,4-dihydroxy-5-(hydroxymethyl)tetrahydrofuran-2yl)-9H-purin-6-yl)amino)phenyl)-2-methylbenzenesulfonamide (7). Gray solid; m.p. $142-146{ }^{\circ} \mathrm{C}$; Yield 70\%; ${ }^{1} \mathrm{H}$ NMR (500 $\left.\mathrm{MHz}, \mathrm{DMSO}-d_{6}\right) \delta 10.28$ (s, $\left.1 \mathrm{H},-\mathrm{SO}_{2}-\mathrm{NH}-\right), 9.86$ (s, $1 \mathrm{H}, \mathrm{Pu}-8$ 'H), 8.50 (s, 1H, Ph-NH), 8.29 (s, 1H, Pu-2’H), 7.82 (d, $J=7.7 \mathrm{~Hz}$, 1H, Ph-H), 7.70 (d, $J=8.8 \mathrm{~Hz}, 2 \mathrm{H}, \mathrm{Ph}-\mathrm{H}), 7.45$ (t, $J=7.8 \mathrm{~Hz}, 1 \mathrm{H}$, Ph-H), $7.34-7.29$ (m, 2H, Ph-H), 7.00 (d, $J=8.9$ Hz, 2H, Ph-H), 5.90 (d, J=6.1 Hz, $1 \mathrm{H}$, ribose 2'-CH), $5.54(\mathrm{~d}, J=6.1 \mathrm{~Hz}, 1 \mathrm{H}$, ribose 3'-CH), $5.34-5.26(\mathrm{~m}, 2 \mathrm{H}$, ribose 5' $\left.-\mathrm{CH}_{2}-\right), 4.58$ (q, $J=5.7 \mathrm{~Hz}, 1 \mathrm{H}$, ribose 5'-CH), 4.14 (q, $J=4.2 \mathrm{~Hz}, 1 \mathrm{H}$, ribose 3'-OH), $3.94(\mathrm{q}, J=3.7 \mathrm{~Hz}, 1 \mathrm{H}$, ribose 4'-CH), 3.64 (dt, $J=12.3,4.3 \mathrm{~Hz}, 1 \mathrm{H}$, ribose 4'-OH), 3.52 (tt, $J=7.2,3.8 \mathrm{~Hz}, 1 \mathrm{H}$, ribose 5'-OH), $2.56\left(\mathrm{~s}, 3 \mathrm{H}, \mathrm{Ph}_{-} \mathrm{CH}_{3}\right) \cdot{ }^{13} \mathrm{C}$ NMR $(125 \mathrm{MHz}$, DMSO- $\left.d_{6}\right) \delta 152.50,152.46,149.79,141.19,138.24,137.32,136.21,133.46,133.09$, $132.84,129.95,126.83,122.26,120.64,88.29,86.39,74.18,71.09,62.04,20.39$. HRMS (ESI): calcd for $\mathrm{C}_{23} \mathrm{H}_{25} \mathrm{O}_{6} \mathrm{~N}_{6} \mathrm{~S}\left([\mathrm{M}+\mathrm{H}]^{+}\right), 513.14780$; found, 513.15393.

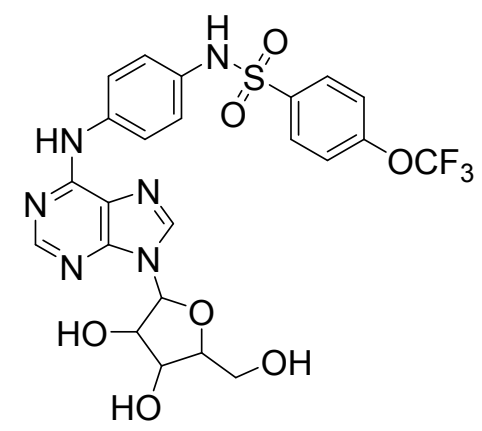

$\mathrm{N}$-(4-((9-(3,4-dihydroxy-5-(hydroxymethyl)tetrahyd
rofuran-2-yl)-9H-purin-6-yl)amino)phenyl)-4-(triflu
oromethoxy)benzenesulfonamide (8). White solid; m.p. $158-160{ }^{\circ} \mathrm{C}$; Yield $80 \%$; ${ }^{1} \mathrm{H}$ NMR $(500 \mathrm{MHz}$, DMSO- $\left.d_{6}\right) \delta 10.28\left(\mathrm{~s}, 1 \mathrm{H},-\mathrm{SO}_{2}-\mathrm{NH}-\right), 9.90(\mathrm{~s}, 1 \mathrm{H}$, Pu-8'H), 8.50 (s, 1H, Ph-NH), 8.33 (s, 1H, Pu-2'H), $7.85(\mathrm{~d}, J=3.8 \mathrm{~Hz}, 2 \mathrm{H}, \mathrm{Ph}-\mathrm{H}), 7.78(\mathrm{~d}, J=9.0 \mathrm{~Hz}, 2 \mathrm{H}, \mathrm{Ph}-\mathrm{H}), 7.54$ (d, $J=4.1 \mathrm{~Hz}$, 2H, Ph-H), 7.03 (d, $J=5.2 \mathrm{~Hz}, 2 \mathrm{H}, \mathrm{Ph}-\mathrm{H}), 5.92$ (d, $J=5.3 \mathrm{~Hz}, 1 \mathrm{H}$, ribose2'-CH), $5.49\left(\mathrm{~d}, J=4.3 \mathrm{~Hz}, 1 \mathrm{H}\right.$, ribose 3'-CH), $5.29-5.21\left(\mathrm{~m}, 2 \mathrm{H}\right.$, ribose 5'-CH $\left.{ }^{-}\right), 4.59(\mathrm{t}, J$ $=5.4 \mathrm{~Hz}, 1 \mathrm{H}$, ribose 5' $-\mathrm{CH}), 4.16-4.12(\mathrm{~m}, 1 \mathrm{H}$, ribose 3'-OH), $3.94(\mathrm{t}, J=4.1 \mathrm{~Hz}$, $1 \mathrm{H}$, ribose4'-CH), $3.68-3.62(\mathrm{~m}, 1 \mathrm{H}$, ribose4'-OH), $3.53(\mathrm{td}, J=8.0,7.5,3.5 \mathrm{~Hz}$, 1H, ribose5'-OH). ${ }^{13} \mathrm{C}$ NMR (125 MHz, DMSO-d $) \delta 152.47,152.41,151.49$, $149.85,141.21,139.11,137.08,132.38,129.90,122.20,122.07,121.89,120.77$, 119.32, 88.33, 86.38, 74.19, 71.07, 62.05. HRMS (ESI): calcd for $\mathrm{C}_{23} \mathrm{H}_{22} \mathrm{O}_{7} \mathrm{~N}_{6} \mathrm{~F}_{3} \mathrm{~S}$ $\left([\mathrm{M}+\mathrm{H}]^{+}\right), 583.11445 ;$ found, 583.12048. 


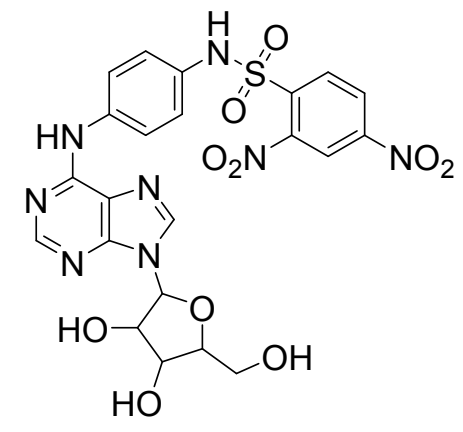

N-(4-((9-(3,4-dihydroxy-5-(hydroxymethyl)tetrahydro furan-2-yl)-9H-purin-6-yl)amino)phenyl)-2,4-dinitrob

enzenesulfonamide (9 ). Yellow solid; m.p. $182-184{ }^{\circ} \mathrm{C}$; Yield 73\%; ${ }^{1} \mathrm{H}$ NMR (500 MHz, DMSO- $\left.d_{6}\right) \delta 11.20$ (s, $\left.1 \mathrm{H},-\mathrm{SO}_{2}-\mathrm{NH}-\right), 10.06\left(\mathrm{~s}, 1 \mathrm{H}, \mathrm{Pu}-8^{\prime} \mathrm{H}\right), 8.94(\mathrm{~s}, 1 \mathrm{H}$, Ph-NH), 8.69 (d, $J=8.7 \mathrm{~Hz}, 1 \mathrm{H}, \mathrm{Ph}-\mathrm{H}), 8.62(\mathrm{~s}, 1 \mathrm{H}$, Pu-2'H), 8.44 (d, $J=3.4$ Hz, 1H, Ph-H), 8.39 (d, $J=8.8$ Hz, 1H, Ph-H), 7.94 (d, $J=$ $5.8 \mathrm{~Hz}, 2 \mathrm{H}, \mathrm{Ph}-\mathrm{H}), 7.20$ (d, $J=5.6 \mathrm{~Hz}, 2 \mathrm{H}, \mathrm{Ph}-\mathrm{H}), 6.02(\mathrm{~d}, J=2.8 \mathrm{~Hz}, 1 \mathrm{H}$, ribose2'-CH), $5.63(\mathrm{~d}, J=3.4 \mathrm{~Hz}, 1 \mathrm{H}$, ribose3'-CH), $5.41-5.35$ (m, 2H, ribose 5' $\left.-\mathrm{CH}_{2}-\right), 4.72-4.67(\mathrm{~m}, 1 \mathrm{H}$, ribose 5' $-\mathrm{CH}), 4.27-4.23(\mathrm{~m}, 1 \mathrm{H}$, ribose 3'-OH), $4.05(\mathrm{t}, J=3.6 \mathrm{~Hz}, 1 \mathrm{H}$, ribose4'-CH), $3.77-3.73(\mathrm{~m}, 1 \mathrm{H}$, ribose4'-OH), $3.64(\mathrm{tt}, J=$ 6.9, $3.2 \mathrm{~Hz}, 1 \mathrm{H}$, ribose5'-OH). ${ }^{13} \mathrm{C}$ NMR (125 MHz, DMSO-d $\left.d_{6}\right) \delta 162.88,152.40$, $150.47,149.92$, 148.46, 141.31, 137.93, 137.14, 132.23, 130.79, 127.70, 123.16, 122.03, 120.72, 88.32, 86.39, 74.22, 71.07, 62.04, 60.30. HRMS (ESI): calcd for $\mathrm{C}_{22} \mathrm{H}_{21} \mathrm{O}_{10} \mathrm{~N}_{8} \mathrm{~S}\left([\mathrm{M}+\mathrm{H}]^{+}\right), 589.10231$; found, 589.10797.

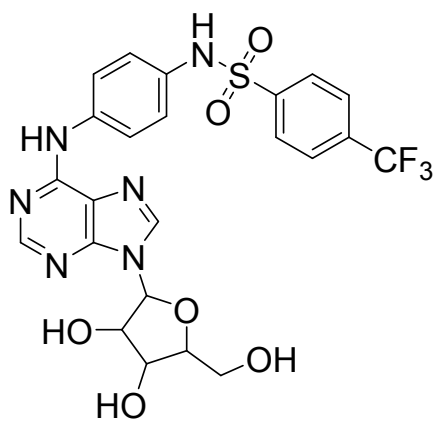

$\boldsymbol{N}$-(4-((9-(3,4-dihydroxy-5-(hydroxymethyl)tetrahydro
furan-2-yl)-9H-purin-6-yl)amino)phenyl)-4-(trifluoro
methyl)benzenesulfonamide $\mathbf{( 1 0 )}$. White solid; m.p.
$153-155^{\circ} \mathrm{C}$. Yield: $77 \% ;{ }^{1} \mathrm{H} \mathrm{NMR}\left(500 \mathrm{MHz}, \mathrm{DMSO}-d_{6}\right)$
$\delta 10.36\left(\mathrm{~s}, 1 \mathrm{H},-\mathrm{SO}_{2}-\mathrm{NH}-\right), 9.96\left(\mathrm{~s}, 1 \mathrm{H}, \mathrm{Pu}-8^{\prime} \mathrm{H}\right), 8.54(\mathrm{~s}$,
$1 \mathrm{H}, \mathrm{Ph}-\mathrm{NH}), 8.36\left(\mathrm{~s}, 1 \mathrm{H}, \mathrm{Pu}-2^{\prime} \mathrm{H}\right), 8.07-7.95(\mathrm{~m}, 4 \mathrm{H}$, Ph-H), 7.82 (d, J=8.8 Hz, 2H, Ph-H), 7.04 (d, $J=8.9$ Hz, 2H, Ph-H), 5.94 (d, $J=6.0$ $\mathrm{Hz}, 1 \mathrm{H}$, ribose 2'-CH), $5.53(\mathrm{~d}, J=6.2 \mathrm{~Hz}, 1 \mathrm{H}$, ribose 3'-CH), $5.34-5.26(\mathrm{~m}, 2 \mathrm{H}$, ribose 5' $\left.-\mathrm{CH}_{2}-\right), 4.62(\mathrm{q}, J=5.9 \mathrm{~Hz}, 1 \mathrm{H}$, ribose 5'-CH), $4.17(\mathrm{td}, J=4.6,3.1 \mathrm{~Hz}, 1 \mathrm{H}$, ribose 3'-OH), 3.97 (q, $J=3.7 \mathrm{~Hz}, 1 \mathrm{H}$, ribose 4'-CH), 3.68 (dt, $J=12.1,4.4 \mathrm{~Hz}, 1 \mathrm{H}$, ribose 4'-OH), $3.59-3.54\left(\mathrm{~m}, 1 \mathrm{H}\right.$, ribose 5'-OH). ${ }^{13} \mathrm{C}$ NMR (125 MHz, DMSO- $\left.d_{6}\right) \delta$ $152.44,149.84,141.28,141.18,137.39,132.00,131.45,131.34,130.14,123.79$, $122.81,122.60,122.07,120.80,120.65,88.31,86.38,74.14,71.07,62.05$. HRMS (ESI): calcd for $\mathrm{C}_{23} \mathrm{H}_{22} \mathrm{O}_{6} \mathrm{~N}_{6} \mathrm{~F}_{3} \mathrm{~S}\left([\mathrm{M}+\mathrm{H}]^{+}\right)$, 567.11954; found, 567.12549. 


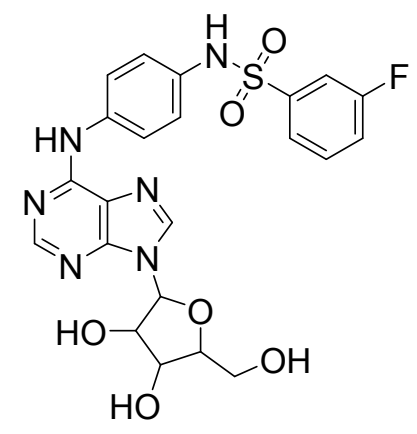

$\mathrm{N}$-(4-((9-(3,4-dihydroxy-5-(hydroxymethyl)tetrahydrofuran -2-yl)-9H-purin-6-yl)amino)phenyl)-3-fluorobenzenesulfona mide (11). White solid; m.p. $140-142{ }^{\circ} \mathrm{C}$. Yield 63\%; ${ }^{1} \mathrm{H}$ NMR $\left(500 \mathrm{MHz}, \mathrm{DMSO}-d_{6}\right) \delta 10.31\left(\mathrm{~s}, 1 \mathrm{H},-\mathrm{SO}_{2}-\mathrm{NH}-\right), 9.92(\mathrm{~s}, 1 \mathrm{H}$, Pu-8'H), 8.50 (s, 1H, Ph-NH), 8.31 (s, 1H, Pu-2'H), 7.76 (d, $J$ $=8.2 \mathrm{~Hz}, 2 \mathrm{H}, \mathrm{Ph}-\mathrm{H}), 7.58-7.52(\mathrm{~m}, 3 \mathrm{H}, \mathrm{Ph}-\mathrm{H}), 7.46(\mathrm{~s}, 1 \mathrm{H}$, Ph-H), 7.02 (d, $J=8.4 \mathrm{~Hz}, 2 \mathrm{H}, \mathrm{Ph}-\mathrm{H}), 5.90$ (d, $J=5.6 \mathrm{~Hz}, 1 \mathrm{H}$, ribose 2'-CH), 5.52 (d, $J$ $=5.6 \mathrm{~Hz}, 1 \mathrm{H}$, ribose 3'-CH), $5.33-5.24\left(\mathrm{~m}, 2 \mathrm{H}\right.$, ribose 5' $\left.-\mathrm{CH}_{2^{-}}\right), 4.59(\mathrm{t}, J=5.6 \mathrm{~Hz}, 1 \mathrm{H}$, ribose 5'-CH), $4.13(\mathrm{t}, J=4.4 \mathrm{~Hz}, 1 \mathrm{H}$, ribose 3'-OH), 3.94 (t, $J=4.4 \mathrm{~Hz}, 1 \mathrm{H}$, ribose 4'-CH), $3.67-3.61$ (m, 1H, ribose 4'-OH), 3.52 (dd, $J=11.9,6.4 \mathrm{~Hz}, 1 \mathrm{H}$, ribose 5'-OH). ${ }^{13} \mathrm{C}$ NMR (125 MHz, DMSO- $\left.d_{6}\right) \delta 163.14,161.16$,152.45, 149.83, 142.13, 141.25, $137.08,132.35,132.18,123.61,122.26,122.12$, 120.76, 120.50, 114.37, 114.18, 88.30, 86.39, 74.16, 71.08, 62.05. HRMS (ESI): calcd for $\mathrm{C}_{22} \mathrm{H}_{22} \mathrm{O}_{6} \mathrm{~N}_{6} \mathrm{FS}\left([\mathrm{M}+\mathrm{H}]^{+}\right), 517.12273$; found, 517.12885.

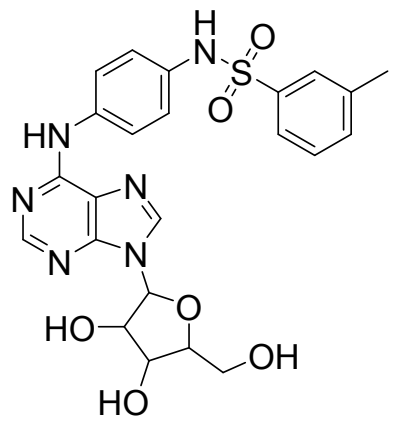

N-(4-((9-(3,4-dihydroxy-5-(hydroxymethyl)tetrahydrofuran2-yl)-9H-purin-6-yl)amino)phenyl)-3-methylbenzenesulfona mide (12). White solid; m.p. $149-151{ }^{\circ} \mathrm{C}$. Yield 65\%; ${ }^{1} \mathrm{H}$ NMR $\left(500 \mathrm{MHz}, \mathrm{DMSO}-d_{6}\right) \delta 10.08\left(\mathrm{~s}, 1 \mathrm{H},-\mathrm{SO}_{2}-\mathrm{NH}-\right), 9.88(\mathrm{~s}, 1 \mathrm{H}$, Pu-8'H), 8.50 (s, 1H, Ph-NH), 8.32 (s, 1H, Pu-2'H), 7.74 (t, $J=$ $7.9 \mathrm{~Hz}, 2 \mathrm{H}, \mathrm{Ph}-\mathrm{H}), 7.57-7.51$ (m, 2H, Ph-H), 7.39 (t, $J=6.6$ Hz, 2H, Ph-H), 7.02 (d, J=7.3 Hz, 2H, Ph-H), 5.92 (d, $J=6.2 \mathrm{~Hz}, 1 \mathrm{H}$, ribose 2'-CH), $5.48\left(\mathrm{~d}, J=6.6 \mathrm{~Hz}, 1 \mathrm{H}\right.$, ribose 3'-CH), $5.30-5.20\left(\mathrm{~m}, 2 \mathrm{H}\right.$, ribose 5'- $\left.\mathrm{CH}_{2}-\right), 4.62-4.57$ (m, 1H, ribose 5'-CH), 4.14 (p, $J=3.4 \mathrm{~Hz}, 1 \mathrm{H}$, ribose 3'-OH), 3.95 (p, J=3.4 Hz, 1H, ribose 4'-CH), $3.68-3.63(\mathrm{~m}, 1 \mathrm{H}$, ribose 4'- $-\mathrm{OH}), 3.54(\mathrm{dt}, J=11.5,5.5 \mathrm{~Hz}, 1 \mathrm{H}$, ribose 5'-OH), 2.32 (s, 3H, Ph-CH3 $).{ }^{13} \mathrm{C}$ NMR (125 MHz, DMSO-d $\left.d_{6}\right) \delta 152.52,152.46,149.80$, $141.23,140.12,139.45,136.67,133.99,132.88,129.60,127.44,124.45,122.20,121.75$, 120.76, 88.33, 86.39, 74.14, 71.09, 62.07, 21.40. HRMS (ESI): calcd for $\mathrm{C}_{23} \mathrm{H}_{25} \mathrm{O}_{6} \mathrm{~N}_{6} \mathrm{~S}$ $\left([\mathrm{M}+\mathrm{H}]^{+}\right), 513.14780$; found, 513.15399. 


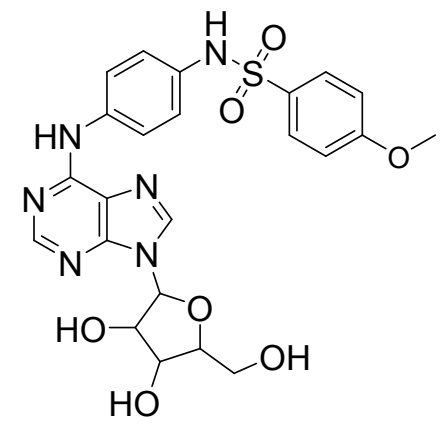

N-(4-((9-(3,4-dihydroxy-5-(hydroxymethyl)tetrahydrofur an-2-yl)-9H-purin-6-yl)amino)phenyl)-4-methoxybenzene sulfonamide (13). Gray solid; m.p. $118-120^{\circ} \mathrm{C}$; Yield $75 \%$; ${ }^{1} \mathrm{H}$ NMR (500 MHz, DMSO-d $\left.d_{6}\right) \delta 10.05$ (s, 1H, -SO $\mathrm{SO}_{2} \mathrm{NH}-$ ), 9.84 (s, 1H, Pu-8'H), 8.50 (s, 1H, Ph-NH), 8.30 (s, 1H, Pu-2’H), $7.74-7.71$ (m, 2H, Ph-H), 7.66 (dd, $J=8.8,1.5$ $\mathrm{Hz}, 2 \mathrm{H}, \mathrm{Ph}-\mathrm{H}), 7.04-7.00$ (m, 4H, Ph-H), 5.91 (d, J=4.7 Hz, 1H, ribose 2'-CH), 5.53 $\left(\mathrm{d}, J=6.1 \mathrm{~Hz}, 1 \mathrm{H}\right.$, ribose 3'-CH), $5.32-5.25\left(\mathrm{~m}, 2 \mathrm{H}\right.$, ribose $\left.5^{\prime}-\mathrm{CH}_{2^{-}}\right), 4.58(\mathrm{q}, J=6.1$ $\mathrm{Hz}, 1 \mathrm{H}$, ribose 5'-CH), $4.15(\mathrm{t}, J=4.3 \mathrm{~Hz}, 1 \mathrm{H}$, ribose 3'-OH), $3.94(\mathrm{~d}, J=3.7 \mathrm{~Hz}, 1 \mathrm{H}$, ribose 4'-CH), 3.75 (s, 3H, $\left.\mathrm{Ph}-\mathrm{OCH}_{3}\right), 3.64$ (dt, $J=12.1,4.4 \mathrm{~Hz}, 1 \mathrm{H}$, ribose4'-OH), 3.55 - 3.51 (m, $1 \mathrm{H}$, ribose 5'-OH). ${ }^{13} \mathrm{C}$ NMR (125 MHz, DMSO- $\left.d_{6}\right) \delta 162.86,152.52,152.46$, $149.79,141.22$, 136.55, 133.11, 131.75, 129.46, 122.15, 121.57, 120.77, 114.88, 88.35, 86.39, 74.12, 71.09, 62.09, 56.13. HRMS (ESI): calcd for $\mathrm{C}_{23} \mathrm{H}_{25} \mathrm{O}_{7} \mathrm{~N}_{6} \mathrm{~S}\left([\mathrm{M}+\mathrm{H}]^{+}\right)$, 529.14272; found, 529.14893.

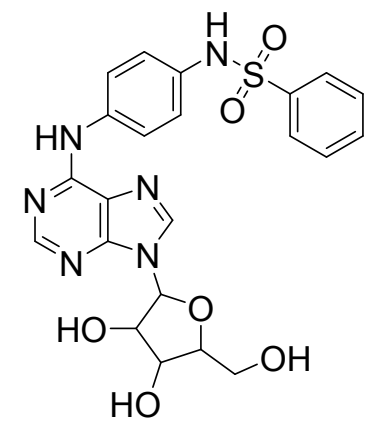

$\mathrm{N}$-(4-((9-(3,4-dihydroxy-5-(hydroxymethyl)tetrahydrofuran -2-yl)-9H-purin-6-yl)amino)phenyl)benzenesulfonamide.

(14). Gray solid; m.p. $138-140{ }^{\circ} \mathrm{C}$; Yield 78\%; ${ }^{1} \mathrm{H}$ NMR (500 $\left.\mathrm{MHz}, \mathrm{DMSO}-d_{6}\right) \delta 10.13\left(\mathrm{~s}, 1 \mathrm{H},-\mathrm{SO}_{2}-\mathrm{NH}-\right), 9.88(\mathrm{~s}, 1 \mathrm{H}$, Pu-8'H), 8.50 (s, 1H, Ph-NH), 8.32 (s, 1H, Pu-2'H), 7.73 (q, $J$ $=7.2 \mathrm{~Hz}, 5 \mathrm{H}, \mathrm{Ph}-\mathrm{H}), 7.52$ (d, $J=7.5 \mathrm{~Hz}, 2 \mathrm{H}, \mathrm{Ph}-\mathrm{H}), 7.01$ (q, $J$ $=7.8,6.0 \mathrm{~Hz}, 2 \mathrm{H}, \mathrm{Ph}-\mathrm{H}), 5.91(\mathrm{~d}, J=5.4 \mathrm{~Hz}, 1 \mathrm{H}$, ribose 2'-CH), 5.49 (d, $J=5.6 \mathrm{~Hz}$, $1 \mathrm{H}$, ribose 3'-CH), $5.32-5.19\left(\mathrm{~m}, 2 \mathrm{H}\right.$, ribose $\left.5^{\prime}-\mathrm{CH}_{2}-\right), 4.59(\mathrm{~d}, J=5.7 \mathrm{~Hz}, 1 \mathrm{H}$, ribose 5 ' $-\mathrm{CH}), 4.14\left(\mathrm{~s}, 1 \mathrm{H}\right.$, ribose $\left.3^{\prime}-\mathrm{OH}\right), 3.94(\mathrm{dt}, J=7.0,3.8 \mathrm{~Hz}, 1 \mathrm{H}$, ribose 4'-CH), 3.64 (d, $J=10.5 \mathrm{~Hz}, 1 \mathrm{H}$, ribose 4'-OH), 3.53 (d, $J=10.7 \mathrm{~Hz}, 1 \mathrm{H}$, ribose 5 '-OH). ${ }^{13} \mathrm{C}$ NMR (125 MHz, DMSO- $\left.d_{6}\right) \delta$ 162.88, 152.44, 149.88, 141.22, 139.04, $138.17,137.08,132.45,129.91,129.24,122.26,122.10,120.79,88.35,86.39,74.21$, 71.09, 62.07. HRMS (ESI): calcd for $\mathrm{C}_{22} \mathrm{H}_{23} \mathrm{O}_{6} \mathrm{~N}_{6} \mathrm{~S}\left([\mathrm{M}+\mathrm{H}]^{+}\right)$, 499.13215; found, 499.13840 . 


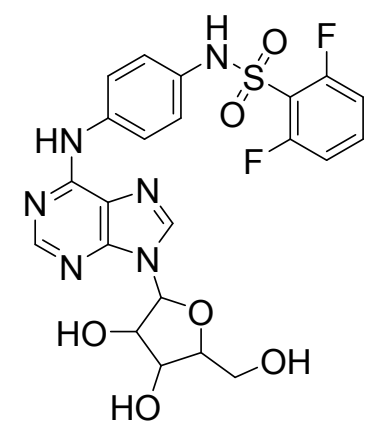

N-(4-((9-(3,4-dihydroxy-5-(hydroxymethyl)tetrahydrofuran -2-yl)-9H-purin-6-yl)amino)phenyl)-2,6-difluorobenzenesul fonamide (15). Gray solid; m.p. $162-164{ }^{\circ} \mathrm{C}$; Yield $67 \% ;{ }^{1} \mathrm{H}$ NMR (500 MHz, DMSO-d $\left.d_{6}\right) \delta 10.44\left(\mathrm{~s}, 1 \mathrm{H},-\mathrm{SO}_{2}-\mathrm{NH}\right), 9.88$ (s, 1H, Pu-8'H), 8.49 (s, 1H, Ph-NH), 8.30 (s, 1H, Pu-2’H), $7.98(\mathrm{dd}, J=8.0,1.6 \mathrm{~Hz}, 1 \mathrm{H}, \mathrm{Ph}-\mathrm{H}), 7.72$ (d, $J=9.0 \mathrm{~Hz}, 2 \mathrm{H}$, Ph-H), 7.60 (t, $J=2.5$ Hz, 1H, Ph-H), $7.49-7.45$ (m, 1H, Ph-H), 7.03 (d, $J=9.0$ Hz, 2H, Ph-H), $5.90(\mathrm{~d}, J=6.1 \mathrm{~Hz}, 1 \mathrm{H}$, ribose 2'-CH), 5.49 (d, $J=6.1 \mathrm{~Hz}, 1 \mathrm{H}$, ribose $\left.3^{\prime}-\mathrm{CH}\right), 5.30-5.22\left(\mathrm{~m}, 2 \mathrm{H}\right.$, ribose $\left.5^{\prime}-\mathrm{CH}_{2}-\right), 4.58(\mathrm{q}, J=5.8 \mathrm{~Hz}, 1 \mathrm{H}$, ribose 5'-CH), $4.12(\mathrm{td}, J=4.8,3.1 \mathrm{~Hz}, 1 \mathrm{H}$, ribose 3'-OH), 3.93 (q, $J=3.6 \mathrm{~Hz}, 1 \mathrm{H}$, ribose 4'-CH), $3.64\left(\mathrm{dt}, J=12.1,4.4 \mathrm{~Hz}, 1 \mathrm{H}\right.$, ribose 4'-OH), $3.56-3.49$ (m, $1 \mathrm{H}$, ribose 5'-OH). ${ }^{13} \mathrm{C}$ NMR (125 MHz, DMSO- $\left.d_{6}\right) \delta 160.47,158.42,152.45,149.84,141.27,137.12$, 136.48, 131.85, 122.22, 121.36, 120.76, 117.20, 114.12, 113.94, 88.30, 86.38, 74.16, 71.08, 62.05. HRMS (ESI): calcd for $\mathrm{C}_{22} \mathrm{H}_{21} \mathrm{O}_{6} \mathrm{~N}_{6} \mathrm{~F}_{2} \mathrm{~S}\left([\mathrm{M}+\mathrm{H}]^{+}\right), 515.11331$; found, 515.11666 .

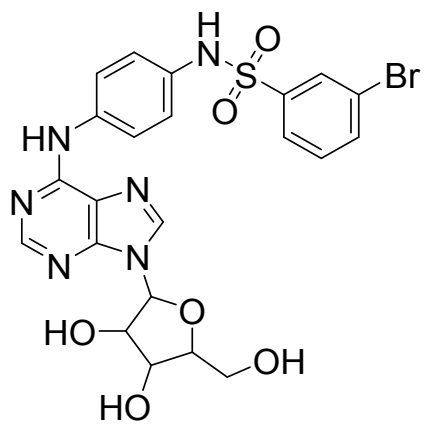

\section{3-bromo- $N$-(4-((9-(3,4-dihydroxy-5-(hydroxymethyl)t} etrahydrofuran-2-yl)-9H-purin-6-yl)amino)phenyl)be nzenesulfonamide (16). Yellow solid; m.p. $135-137{ }^{\circ} \mathrm{C}$; Yield 71\%; ${ }^{1} \mathrm{H}$ NMR $\left(500 \mathrm{MHz}, \mathrm{DMSO}-d_{6}\right) \delta 10.34(\mathrm{~s}$, $\left.1 \mathrm{H},-\mathrm{SO}_{2}-\mathrm{NH}\right), 9.99$ (s, 1H, $\left.\mathrm{Pu}-8^{\prime} \mathrm{H}\right), 8.57$ (s, 1H, Ph-NH), 8.39 (s, 1H, Pu-2'H), 7.93 (d, $J=2.0 \mathrm{~Hz}, 1 \mathrm{H}$, Ph-H), $7.86-7.83$ (m, 2H, Ph-H), 7.75 (d, J=7.9 Hz, 1H, Ph-H), 7.55 (dd, J= 7.9, $2.1 \mathrm{~Hz}, 1 \mathrm{H}, \mathrm{Ph}-\mathrm{H}), 7.08$ (d, $J=8.8 \mathrm{~Hz}, 2 \mathrm{H}, \mathrm{Ph}-\mathrm{H}), 5.97$ (d, $J=6.0 \mathrm{~Hz}, 1 \mathrm{H}$, ribose 2'-CH), $5.57(\mathrm{~d}, J=6.0 \mathrm{~Hz}, 1 \mathrm{H}$, ribose 3'- $\mathrm{CH}), 5.37-5.27$ (m, $2 \mathrm{H}$, ribose 5' $\left.-\mathrm{CH}_{2}-\right)$, $4.70-4.62\left(\mathrm{~m}, 1 \mathrm{H}\right.$, ribose $\left.5^{\prime}-\mathrm{CH}\right), 4.20(\mathrm{~d}, J=4.1 \mathrm{~Hz}, 1 \mathrm{H}$, ribose 3 ' $-\mathrm{OH}), 4.00(\mathrm{~d}, J$ $=3.7 \mathrm{~Hz}, 1 \mathrm{H}$, ribose 4'-CH), 3.71 (dt, $J=13.9,4.6 \mathrm{~Hz}, 1 \mathrm{H}$, ribose 4'-OH), $3.63-$ $3.57\left(\mathrm{~m}, 1 \mathrm{H}\right.$, ribose $\left.5{ }^{\prime}-\mathrm{OH}\right) .{ }^{13} \mathrm{C} \mathrm{NMR}\left(125 \mathrm{MHz}, \mathrm{DMSO}-d_{6}\right) \delta 152.46,149.84$, $142.07,141.26,137.15,136.22,132.27,132.05,129.63,126.38,122.60,122.35$, 122.12, 120.75, 88.29, 86.38, 74.18, 71.07, 62.04. HRMS (ESI): calcd for $\mathrm{C}_{22} \mathrm{H}_{22} \mathrm{O}_{6} \mathrm{~N}_{6} \mathrm{BrS}\left([\mathrm{M}+\mathrm{H}]^{+}\right), 577.04267$; found, 577.04865. 


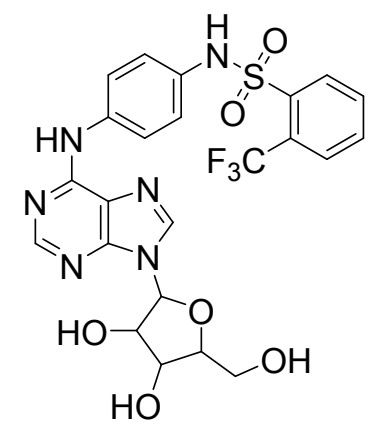

N-(4-((9-(3,4-dihydroxy-5-(hydroxymethyl)tetrahydrofura n-2-yl)-9H-purin-6-yl)amino)phenyl)-2-(trifluoromethyl)be nzenesulfonamide (17). Yellow solid; m.p. $134-136{ }^{\circ} \mathrm{C}$; Yield 66\%; ${ }^{1} \mathrm{H}$ NMR (500 MHz, DMSO- $\left.d_{6}\right) \delta 10.58(\mathrm{~s}, 1 \mathrm{H}$, - $\left.\mathrm{SO}_{2}-\mathrm{NH}\right), 9.94$ (s, $\left.1 \mathrm{H}, \mathrm{Pu}-8^{\prime} \mathrm{H}\right), 8.54$ (s, 1H, Ph-NH), 8.35 (s, 1H, Pu-2'H), 8.11 (d, $J=7.9 \mathrm{~Hz}, 1 \mathrm{H}, \mathrm{Ph}-\mathrm{H}), 7.99$ (d, $J=7.7$ Hz, 1H, Ph-H), $7.88-7.79$ (m, 4H, Ph-H), 7.08 (d, J= 7.1 Hz, 2H, Ph-H), 5.94 (d, $J$ $=6.0 \mathrm{~Hz}, 1 \mathrm{H}$, ribose 2' $-\mathrm{CH}), 5.58-5.52\left(\mathrm{~m}, 1 \mathrm{H}\right.$, ribose $\left.3^{\prime}-\mathrm{CH}\right), 5.31(\mathrm{dt}, J=24.6$, $4.1 \mathrm{~Hz}, 2 \mathrm{H}$, ribose 5'- $\left.\mathrm{CH}_{2}{ }^{-}\right), 4.62(\mathrm{~d}, J=5.8 \mathrm{~Hz}, 1 \mathrm{H}$, ribose 5' $-\mathrm{CH}), 4.17$ (q, $J=3.8$, $3.0 \mathrm{~Hz}, 1 \mathrm{H}$, ribose 3'-OH), 3.97 (t, $J=3.3 \mathrm{~Hz}, 1 \mathrm{H}$, ribose 4'-CH), 3.68 (dt, $J=12.8$, $4.2 \mathrm{~Hz}, 1 \mathrm{H}$, ribose 4'-OH), $3.59-3.53\left(\mathrm{~m}, 1 \mathrm{H}\right.$, ribose 5'-OH). ${ }^{13} \mathrm{C}$ NMR (125 MHz, DMSO- $\left.d_{6}\right) \delta 152.44,149.84,141.28,140.18,137.39,132.00,131.45,131.34,130.14$, $123.79,122.60,121.07,120.80,88.31,86.38,74.14,71.07,62.05$. HRMS (ESI): calcd for $\mathrm{C}_{23} \mathrm{H}_{22} \mathrm{O}_{6} \mathrm{~N}_{6} \mathrm{~F}_{3} \mathrm{~S}\left([\mathrm{M}+\mathrm{H}]^{+}\right)$, 567.11954; found, 567.12543.

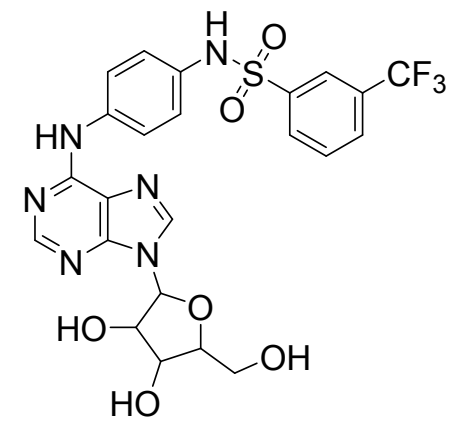

N-(4-((9-(3,4-dihydroxy-5-(hydroxymethyl)tetrahydro furan-2-yl)-9H-purin-6-yl)amino)phenyl)-3-(trifluoro methyl)benzenesulfonamide (18). Yellow solid; m.p. 164-166 ${ }^{\circ} \mathrm{C}$; Yield: 68\%; ${ }^{1} \mathrm{H}$ NMR $\quad(500 \mathrm{MHz}$, DMSO-d $\left.d_{6}\right) \delta 10.36\left(\mathrm{~s}, 1 \mathrm{H},-\mathrm{SO}_{2}-\mathrm{NH}\right), 9.96(\mathrm{~s}, 1 \mathrm{H}$, Pu-8'H), 8.54 (s, 1H, Ph-NH), 8.36 (s, 1H, Pu-2'H), 8.14 - 7.92 (m, 4H, Ph-H), 7.82 (d, J=8.8 Hz, 2H, Ph-H), 7.04 (d, J=8.9 Hz, 2H, Ph-H), $5.94(\mathrm{~d}, J=6.1 \mathrm{~Hz}, 1 \mathrm{H}$, ribose 2'-CH), 5.53 (d, $J=6.2 \mathrm{~Hz}, 1 \mathrm{H}$, ribose 3'-CH), 5.33 $5.26\left(\mathrm{~m}, 2 \mathrm{H}\right.$, ribose 5'- $\left.\mathrm{CH}_{2}-\right), 4.62(\mathrm{q}, J=5.9 \mathrm{~Hz}, 1 \mathrm{H}$, ribose 5' $-\mathrm{CH}), 4.17(\mathrm{td}, J=4.6$, $3.1 \mathrm{~Hz}, 1 \mathrm{H}$, ribose 3'-OH), 3.97 (q, $J=3.7 \mathrm{~Hz}, 1 \mathrm{H}$, ribose 4'- $\mathrm{CH}$ ), 3.68 (dt, $J=12.1$, $4.4 \mathrm{~Hz}, 1 \mathrm{H}$, ribose 4'-OH), 3.56 (ddd, $J=12.1,6.8,3.8 \mathrm{~Hz}, 1 \mathrm{H}$, ribose $\left.5^{\prime}-\mathrm{OH}\right) .{ }^{13} \mathrm{C}$ NMR (125 MHz, DMSO- $\left.d_{6}\right) \delta 152.44,149.84,143.28,141.28,140.18,137.39$, $134.00,133.45,131.34,130.14,123.79,122.60,121.07,120.80,88.31,86.38,74.14$, 71.07, 62.05. HRMS (ESI): calcd for $\mathrm{C}_{23} \mathrm{H}_{22} \mathrm{O}_{6} \mathrm{~N}_{6} \mathrm{~F}_{3} \mathrm{~S}\left([\mathrm{M}+\mathrm{H}]^{+}\right), 567.11954$; found, 567.121537 . 


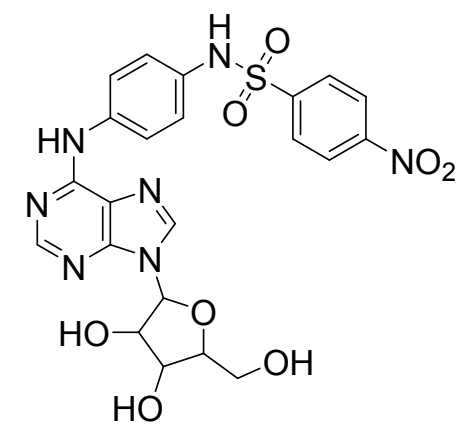

N-(4-((9-(3,4-dihydroxy-5-(hydroxymethyl)tetrahydro furan-2-yl)-9H-purin-6-yl)amino)phenyl)-4-nitrobenz enesulfonamide (19). Yellow solid; m.p. $134-136{ }^{\circ} \mathrm{C}$; Yield 78\%; ${ }^{1} \mathrm{H}$ NMR (500 MHz, DMSO- $\left.d_{6}\right) \delta 10.54(\mathrm{~s}$, 1H, $\left.\mathrm{SO}_{2}-\mathrm{NH}\right), 9.95$ (s, 1H, Pu-8'H), 8.51 (s, 1H, Pu-2’H), 8.34 (d, $J=8.6 \mathrm{~Hz}, 2 \mathrm{H}, \mathrm{Ph}-\mathrm{H}), 8.11$ (s, 1H, Ph-NH), 7.97 (d, $J=8.6 \mathrm{~Hz}, 2 \mathrm{H}, \mathrm{Ph}-\mathrm{H}), 7.83-7.73$ (m, 2H, Ph-H), 7.04 (d, J=4.1 Hz, 2H, Ph-H), $5.90(\mathrm{~d}, J=5.5 \mathrm{~Hz}, 1 \mathrm{H}$, ribose 2'-CH), 5.53 (t, $J=4.9 \mathrm{~Hz}, 1 \mathrm{H}$, ribose 3'-CH), 5.31 $5.23\left(\mathrm{~m}, 2 \mathrm{H}\right.$, ribose 5' $\left.-\mathrm{CH}_{2^{-}}\right), 4.59(\mathrm{t}, J=5.5 \mathrm{~Hz}, 1 \mathrm{H}$, ribose 5'- $\mathrm{CH}), 4.16-4.09(\mathrm{~m}$, $1 \mathrm{H}$, ribose 3'-OH), $3.94(\mathrm{q}, J=4.5,3.6 \mathrm{~Hz}, 1 \mathrm{H}$, ribose 4'-CH), $3.67-3.62(\mathrm{~m}, 1 \mathrm{H}$, ribose4'-OH), $3.55-3.50(\mathrm{~m}, 1 \mathrm{H}$, ribose 5 ' $-\mathrm{OH}) .{ }^{13} \mathrm{C}$ NMR (125 MHz, DMSO- $\left.d_{6}\right) \delta$ $162.88,152.44,149.88,141.22,139.04,138.17,137.08,132.45,129.91,128.24$, 122.26, 122.10, 120.79, 88.35, 86.39, 74.21, 71.09, 62.07. HRMS (ESI): calcd for $\mathrm{C}_{22} \mathrm{H}_{22} \mathrm{O}_{8} \mathrm{~N}_{7} \mathrm{~S}\left([\mathrm{M}+\mathrm{H}]^{+}\right), 544.11723$; found, 544.12317.

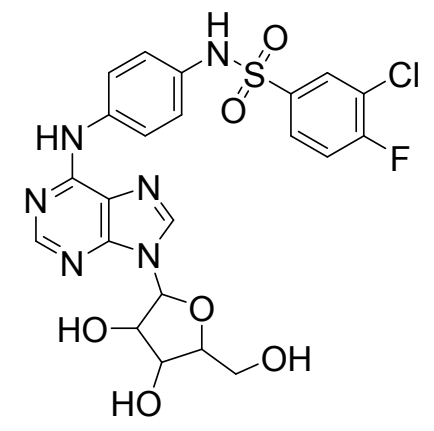

3-chloro- $\mathrm{N}$-(4-((9-(3,4-dihydroxy-5-(hydroxymethyl)tetr
ahydrofuran-2-yl)-9H-purin-6-yl)amino)phenyl)-4-fluor obenzenesulfonamide (20). Gray solid; m.p. $143-145^{\circ} \mathrm{C}$; Yield 72\%; ${ }^{1} \mathrm{H}$ NMR (500 MHz, DMSO- $\left.d_{6}\right) \delta 10.34(\mathrm{~s}, 1 \mathrm{H}$, $\left.-\mathrm{SO}_{2}-\mathrm{NH}\right), 10.00(\mathrm{~s}, 1 \mathrm{H}, \mathrm{Pu}-8$ 'H), 8.57 (s, 1H, Ph-NH), $8.39\left(\mathrm{~d}, J=1.1 \mathrm{~Hz}, 1 \mathrm{H}, \mathrm{Pu}-2^{\prime} \mathrm{H}\right), 7.97$ (d, $J=6.1 \mathrm{~Hz}, 1 \mathrm{H}$, Ph-H), 7.86 (d, J = 9.0 Hz, 2H, Ph-H), $7.79-7.74$ (m, 1H, Ph-H), 7.65 (s, 1H, Ph-H), 7.09 (d, $J=8.8 \mathrm{~Hz}, 2 \mathrm{H}, \mathrm{Ph}-\mathrm{H}), 5.97$ (d, $J=5.8 \mathrm{~Hz}, 1 \mathrm{H}$, ribose 2'-CH), 5.56 (d, $J=6.3$ $\mathrm{Hz}, 1 \mathrm{H}$, ribose 3'-CH), $5.32\left(\mathrm{dd}, J=22.3,5.1 \mathrm{~Hz}, 2 \mathrm{H}\right.$, ribose $\left.5^{\prime}-\mathrm{CH}_{2}-\right), 4.65$ (q, $J=$ $5.7 \mathrm{~Hz}, 1 \mathrm{H}$, ribose 5'-CH), 4.19 (q, $J=4.3 \mathrm{~Hz}, 1 \mathrm{H}$, ribose 3'-OH), 4.00 (q, $J=3.7 \mathrm{~Hz}$, $1 \mathrm{H}$, ribose 4'-CH), 3.71 (dt, $J=12.3,4.3 \mathrm{~Hz}, 1 \mathrm{H}$, ribose 4'-OH), 3.59 (ddd, $J=11.0$, 6.6, $3.3 \mathrm{~Hz}, 1 \mathrm{H}$, ribose 5'-OH). ${ }^{13} \mathrm{C}$ NMR (125 MHz, DMSO- $\left.d_{6}\right) \delta 152.43,149.86$, $147.88,141.30,140.25,137.61,133.73,132.02,131.58,130.36,124.75,122.92$, 122.09, 120.81, 88.30, 86.38, 74.14, 71.07, 62.05. HRMS (ESI): calcd for $\mathrm{C}_{22} \mathrm{H}_{21} \mathrm{O}_{6} \mathrm{~N}_{6} \mathrm{ClFS}\left([\mathrm{M}+\mathrm{H}]^{+}\right), 551.08376$; found, 551.08972 . 


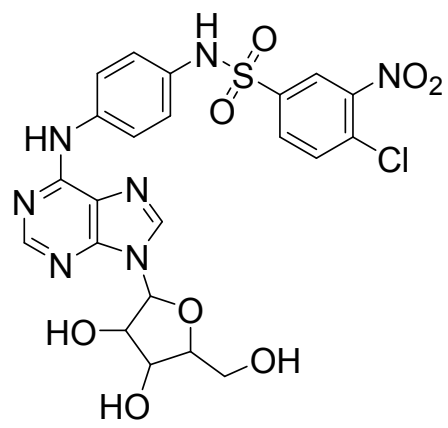

4-chloro- $N$-(4-((9-(3,4-dihydroxy-5-(hydroxymethyl)te trahydrofuran-2-yl)-9H-purin-6-yl)amino)phenyl)-3-n itrobenzenesulfonamide (21). Gray solid; m.p. 143-145 ${ }^{\circ} \mathrm{C}$; Yield: $72 \% .{ }^{1} \mathrm{H}$ NMR $\left(500 \mathrm{MHz}\right.$, DMSO- $\left.d_{6}\right)$ $\delta 10.53\left(\mathrm{~s}, 1 \mathrm{H},-\mathrm{SO}_{2}-\mathrm{NH}\right), 9.96\left(\mathrm{~s}, 1 \mathrm{H}, \mathrm{Pu}-8^{\prime} \mathrm{H}\right), 8.51(\mathrm{~s}$, 1H, Ph-NH), 8.41 (d, $J=1.7 \mathrm{~Hz}, 1 \mathrm{H}, \mathrm{Ph}-\mathrm{H}), 8.33$ (s, 1H, Pu-2'H), 8.10 (d, J = 1.3 Hz, 1H, Ph-H), 7.95 (s, 1H, Ph-H), 7.82 (d, J = 9.0 Hz, 2H, Ph-H), 7.05 (d, $J=8.9 \mathrm{~Hz}, 2 \mathrm{H}, \mathrm{Ph}-\mathrm{H}), 5.91$ (d, $J=6.1 \mathrm{~Hz}, 1 \mathrm{H}$, ribose 2'-CH), 5.51 (d, $J=6.2 \mathrm{~Hz}, 1 \mathrm{H}$, ribose $\left.3^{\prime}-\mathrm{CH}\right), 5.29-5.23\left(\mathrm{~m}, 2 \mathrm{H}\right.$, ribose $\left.5^{\prime}-\mathrm{CH}_{2^{-}}\right), 4.59$ (q, $J=5.8$ $\mathrm{Hz}, 1 \mathrm{H}$, ribose $\left.5^{\prime}-\mathrm{CH}\right), 4.13\left(\mathrm{td}, J=4.7,3.0 \mathrm{~Hz}, 1 \mathrm{H}\right.$, ribose $\left.3^{\prime}-\mathrm{OH}\right), 3.94(\mathrm{q}, J=3.6$ $\mathrm{Hz}, 1 \mathrm{H}$, ribose 4'-CH), $3.64(\mathrm{dt}, J=8.6,4.3 \mathrm{~Hz}, 1 \mathrm{H}$, ribose 4'- $-\mathrm{OH}), 3.55-3.51$ (m, $1 \mathrm{H}$, ribose 5 '-OH). ${ }^{13} \mathrm{C}$ NMR (125 MHz, DMSO- $\left.d_{6}\right) \delta 152.41,149.85,147.87,141.29$, $140.24,138.00,137.60,133.71,132.01,131.57,130.35,124.74,122.90,122.08$, 120.80, 88.29, 86.37, 74.13, 71.06, 62.04. HRMS (ESI): calcd for $\mathrm{C}_{22} \mathrm{H}_{21} \mathrm{O}_{8} \mathrm{~N}_{7} \mathrm{ClS}$ $\left([\mathrm{M}+\mathrm{H}]^{+}\right)$, 578.07826; found, 578.08405.

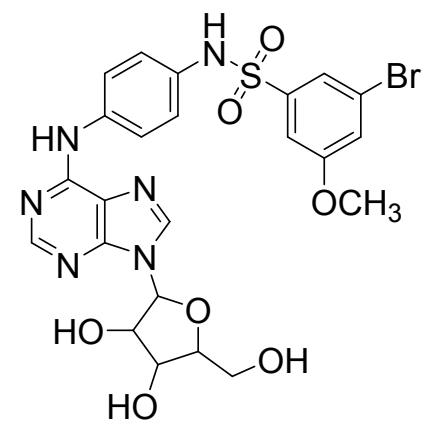

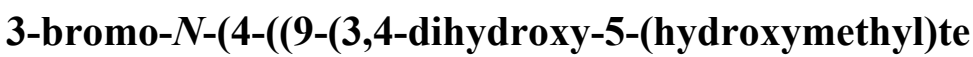
trahydrofuran-2-yl)-9H-purin-6-yl)amino)phenyl)-5-m ethoxybenzenesulfonamide (22). Gray solid; m.p. $148-150{ }^{\circ} \mathrm{C}$; Yield $75 \%$; ${ }^{1} \mathrm{H}$ NMR $\left(500 \mathrm{MHz}, \mathrm{DMSO}-d_{6}\right)$ $\delta: 9.94\left(\mathrm{~s}, 1 \mathrm{H},-\mathrm{SO}_{2}-\mathrm{NH}\right), 9.86(\mathrm{~s}, 1 \mathrm{H}, \mathrm{Pu}-8$ 'H), 8.49 (s, 1H, Ph-NH), $8.31\left(\mathrm{~s}, 1 \mathrm{H}, \mathrm{Pu}-2^{\prime} \mathrm{H}\right), 7.74-7.68$ (m, 4H, Ph-H), 7.15 (dd, $J=8.3,0.9$ Hz, 1H, Ph-H), 7.01 (d, $J=8.9$ Hz, 2H, Ph-H), 5.91 (d, $J=6.1 \mathrm{~Hz}, 1 \mathrm{H}$, ribose 2'-CH), $5.50(\mathrm{~d}, J=6.1 \mathrm{~Hz}, 1 \mathrm{H}$, ribose 3'-CH), $5.30-5.21$ $\left(\mathrm{m}, 2 \mathrm{H}\right.$, ribose $\left.5^{\prime}-\mathrm{CH}_{2}{ }^{-}\right), 4.59\left(\mathrm{q}, J=5.8 \mathrm{~Hz}, 1 \mathrm{H}\right.$, ribose $\left.5^{\prime}-\mathrm{CH}\right), 4.14(\mathrm{td}, J=4.7,3.1$ $\mathrm{Hz}, 1 \mathrm{H}$, ribose 3'-OH), 3.94 (q, $J=3.6 \mathrm{~Hz}, 1 \mathrm{H}$, ribose 4'-CH), 3.89 (s, 3H), 3.65 (dt, $J=12.1,4.3 \mathrm{~Hz}, 1 \mathrm{H}$, ribose $\left.4^{\prime}-\mathrm{OH}\right), 3.57-3.51$ (m, $1 \mathrm{H}$, ribose $\left.5^{\prime}-\mathrm{OH}\right) .{ }^{13} \mathrm{C}$ NMR $\left(125 \mathrm{MHz}, \mathrm{DMSO}-d_{6}\right) \delta 156.21,152.50,149.81,141.23,137.98,136.83,132.46$, $128.79,122.14,121.85,120.75,115.93,111.49,88.34,86.39,74.14,71.09,62.07$, 57.10. HRMS (ESI): calcd for $\mathrm{C}_{23} \mathrm{H}_{24} \mathrm{O}_{7} \mathrm{~N}_{6} \mathrm{BrS}\left([\mathrm{M}+\mathrm{H}]^{+}\right), 607.05323$; found, 607.05859 . 


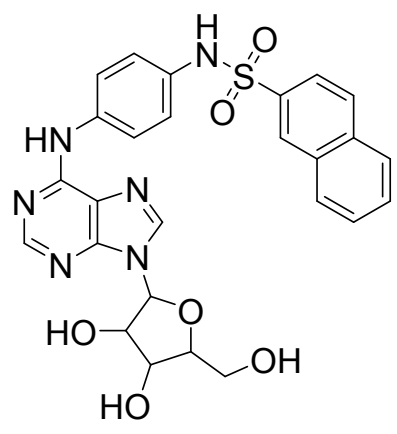

N-(4-((9-(3,4-dihydroxy-5-(hydroxymethyl)tetrahydrofur an-2-yl)-9H-purin-6-yl)amino)phenyl)naphthalene-2-sulf

onamide (23). Gray solid; m.p. $137-139^{\circ} \mathrm{C}$; Yield 74\%;

${ }^{1} \mathrm{H}$ NMR (500 MHz, DMSO- $\left.d_{6}\right) \delta 10.26\left(\mathrm{~s}, 1 \mathrm{H},-\mathrm{SO}_{2}-\mathrm{NH}\right)$, 9.86 (s, 1H, Naph-H), 8.48 (s, 1H, Pu-8'H), 8.39 (s, 1H, Ph-NH), 8.29 (s, 1H, Pu-2'H), 8.08 (dd, $J=13.6,8.4$ Hz, 2H,

Naph-H), 7.97 (d, $J=8.1 \mathrm{~Hz}, 1 \mathrm{H}$, Naph-H), 7.77 (dd, $J=8.7,2.0 \mathrm{~Hz}, 1 \mathrm{H}$, Naph-H), 7.72 (d, $J=8.5 \mathrm{~Hz}, 2 \mathrm{H}, \mathrm{Ph}-\mathrm{H}), 7.65-7.57$ (m, 2H, Naph-H), 7.04 (d, $J=8.8 \mathrm{~Hz}, 2 \mathrm{H}$, Ph-H), 5.89 (d, $J=6.1 \mathrm{~Hz}, 1 \mathrm{H}$, ribose 2'-CH), 5.49 (d, $J=6.2 \mathrm{~Hz}, 1 \mathrm{H}$, ribose 3'-CH), $5.30-5.21\left(\mathrm{~m}, 2 \mathrm{H}\right.$, ribose 5' $\left.-\mathrm{CH}_{2}-\right), 4.58$ (q, $J=5.8 \mathrm{~Hz}, 1 \mathrm{H}$, ribose $\left.5^{\prime}-\mathrm{CH}\right), 4.12$ (q, $J$ $=4.2 \mathrm{~Hz}, 1 \mathrm{H}$, ribose $\left.3^{\prime}-\mathrm{OH}\right), 3.93(\mathrm{q}, J=3.5 \mathrm{~Hz}, 1 \mathrm{H}$, ribose 4' $-\mathrm{CH}), 3.68-3.60(\mathrm{~m}$, $1 \mathrm{H}$, ribose $\left.4^{\prime}-\mathrm{OH}\right), 3.56-3.49\left(\mathrm{~m}, 1 \mathrm{H}\right.$, ribose $\left.5^{\prime}-\mathrm{OH}\right) .{ }^{13} \mathrm{C}$ NMR $(125 \mathrm{MHz}$, DMSO- $\left.d_{6}\right) \delta 152.44,141.22,137.05,136.71,134.74,132.72,132.06,129.96,129.75$, $129.51,128.47,128.39,128.26,122.68,122.13,120.69,88.32,86.37,74.10,71.06$, 62.05. HRMS (ESI): calcd for $\mathrm{C}_{26} \mathrm{H}_{25} \mathrm{O}_{6} \mathrm{~N}_{6} \mathrm{~S}\left([\mathrm{M}+\mathrm{H}]^{+}\right)$, 549.14780; found, 549.15369.

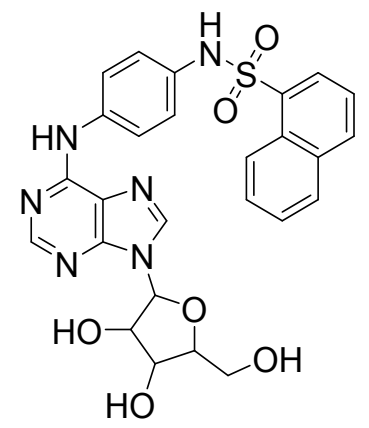
N-(4-((9-(3,4-dihydroxy-5-(hydroxymethyl)tetrahydrofura n-2-yl)-9H-purin-6-yl)amino)phenyl)naphthalene-1-sulfon amide (24). Gray solid; m.p. 133-135 ${ }^{\circ} \mathrm{C}$; Yield 64\%; ${ }^{1} \mathrm{H}$ NMR (500 MHz, DMSO- $\left.d_{6}\right) \delta 10.53\left(\mathrm{~s}, 1 \mathrm{H},-\mathrm{SO}_{2}-\mathrm{NH}\right), 9.81$ (s, 1H, Pu-8'H), 8.72 (d, $J=8.7 \mathrm{~Hz}, 1 \mathrm{H}$, Naph-H), 8.47 (s, 1H, Ph-NH), 8.26 (s, 1H, Pu-2'H), 8.16 (d, $J=7.0 \mathrm{~Hz}, 2 \mathrm{H}$, Naph-H), 8.04 (d, $J=8.2$ Hz, 1H, Naph-H), 7.71 (d, $J=8.0 \mathrm{~Hz}, 1 \mathrm{H}$, Naph-H), 7.64 (d, $J=7.4 \mathrm{~Hz}, 2 \mathrm{H}, \mathrm{Ph}-\mathrm{H}), 7.58$ (td, $J=7.9,2.0 \mathrm{~Hz}, 2 \mathrm{H}, \mathrm{Naph}-\mathrm{H}), 6.92$ (d, $J=8.5$ $\mathrm{Hz}, 2 \mathrm{H}, \mathrm{Ph}-\mathrm{H}), 5.89$ (d, $J=6.1 \mathrm{~Hz}, 1 \mathrm{H}$, ribose $\left.2^{\prime}-\mathrm{CH}\right), 5.48$ (d, $J=6.0 \mathrm{~Hz}, 1 \mathrm{H}$, ribose 3'-CH), $5.31-5.19\left(\mathrm{~m}, 2 \mathrm{H}\right.$, ribose 5' $\left.-\mathrm{CH}_{2}{ }^{-}\right), 4.57$ (d, $J=5.8 \mathrm{~Hz}, 1 \mathrm{H}$, ribose 5'-CH), $4.16-4.07(\mathrm{~m}, 1 \mathrm{H}$, ribose 3'-OH), $3.93(\mathrm{~d}, J=3.6 \mathrm{~Hz}, 1 \mathrm{H}$, ribose 4'-CH), $3.64\left(\mathrm{~d}, J=12.1 \mathrm{~Hz}, 1 \mathrm{H}\right.$, ribose 4'-OH), $3.54-3.48\left(\mathrm{~m}, 1 \mathrm{H}\right.$, ribose $\left.5^{\prime}-\mathrm{OH}\right) .{ }^{13} \mathrm{C}$ NMR (125 MHz, DMSO-d $\left.d_{6}\right) \delta$ 152.43, 149.69, 141.18, 136.16, 134.92, 134.24, $132.75,130.53,129.63,128.73,127.97,127.56,125.04,124.88,122.18,120.67$, 88.28, 86.37, 74.12, 71.04, 62.02. HRMS (ESI): calcd for $\mathrm{C}_{26} \mathrm{H}_{25} \mathrm{O}_{6} \mathrm{~N}_{6} \mathrm{~S}\left([\mathrm{M}+\mathrm{H}]^{+}\right)$, 
549.14980; found, 549.15387.

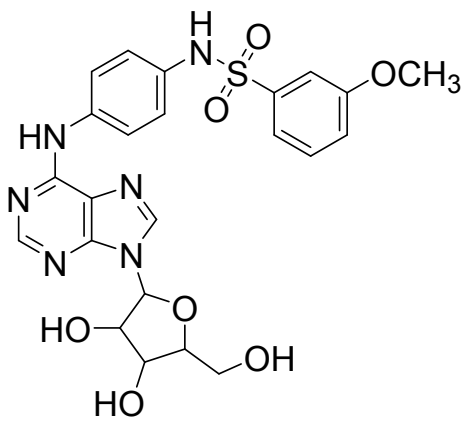

N-(4-((9-(3,4-dihydroxy-5-(hydroxymethyl)tetrahydr ofuran-2-yl)-9H-purin-6-yl)amino)phenyl)-3-methox ybenzenesulfonamide (25). Gray solid; m.p. $173-175{ }^{\circ} \mathrm{C}$; Yield: $66 \%$; ${ }^{1} \mathrm{H}$ NMR $(500 \mathrm{MHz}$, DMSO- $\left.d_{6}\right) \delta 10.14\left(\mathrm{~s}, 1 \mathrm{H},-\mathrm{SO}_{2}-\mathrm{NH}\right), 9.89(\mathrm{~s}, 1 \mathrm{H}$, Pu-8'H), 8.49 (s, 1H, Ph-NH), 8.31 (s, 1H, Pu-2’H), $7.74(\mathrm{~d}, J=7.1 \mathrm{~Hz}, 2 \mathrm{H}, \mathrm{Ph}-\mathrm{H}), 7.42$ (td, $J=8.0,1.8 \mathrm{~Hz}, 1 \mathrm{H}, \mathrm{Ph}-\mathrm{H}), 7.29-7.22$ (m, 2H, Ph-H), 7.13 (dt, $J=8.4,2.2 \mathrm{~Hz}, 1 \mathrm{H}, \mathrm{Ph}-\mathrm{H}), 7.02$ (d, $J=6.9 \mathrm{~Hz}, 2 \mathrm{H}, \mathrm{Ph}-\mathrm{H}), 5.90$ (d, $J=1.8 \mathrm{~Hz}, 1 \mathrm{H}$, ribose 2'-CH), $5.50(\mathrm{~d}, J=1.8 \mathrm{~Hz}, 1 \mathrm{H}$, ribose 3'-CH), $5.32-5.21$ $\left(\mathrm{m}, 2 \mathrm{H}\right.$, ribose $\left.5^{\prime}-\mathrm{CH}_{2}-\right), 4.58\left(\mathrm{q}, J=6.4,5.5 \mathrm{~Hz}, 1 \mathrm{H}\right.$, ribose $\left.5^{\prime}-\mathrm{CH}\right), 4.13(\mathrm{t}, J=4.4$ $\mathrm{Hz}, 1 \mathrm{H}$, ribose 3'-OH), $3.94(\mathrm{dd}, J=3.6,1.8 \mathrm{~Hz}, 1 \mathrm{H}$, ribose 4'-CH), $3.73(\mathrm{~s}, 3 \mathrm{H}$, $\left.\mathrm{Ph}^{-} \mathrm{OCH}_{3}\right), 3.67-3.62\left(\mathrm{~m}, 1 \mathrm{H}\right.$, ribose 4'-OH), $3.55-3.50(\mathrm{~m}, 1 \mathrm{H}$, ribose 5 ' $-\mathrm{OH}) .{ }^{13} \mathrm{C}$ NMR (125 MHz, DMSO- $\left.d_{6}\right) \delta 159.82,152.50,152.45,149.81,141.30,141.23$, $136.80,132.80,130.95,122.19,121.97,120.75,119.35,119.13,112.23,88.31,86.38$, 74.13, 71.08, 62.06, 56.09. HRMS (ESI): calcd for $\mathrm{C}_{23} \mathrm{H}_{25} \mathrm{ClO}_{7} \mathrm{~N}_{6} \mathrm{~S}\left([\mathrm{M}+\mathrm{H}]^{+}\right)$, 529.14272; found, 529.14872. 


\section{3. ${ }^{1} \mathrm{H},{ }^{13} \mathrm{C}$ NMR, HRMS data}

3.1. ${ }^{1} \mathrm{H},{ }^{13} \mathrm{C}$ NMR data of intermediates (A)

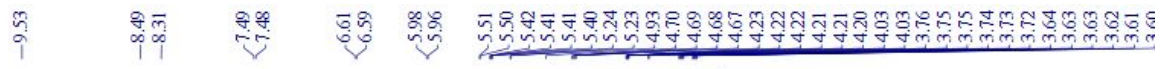
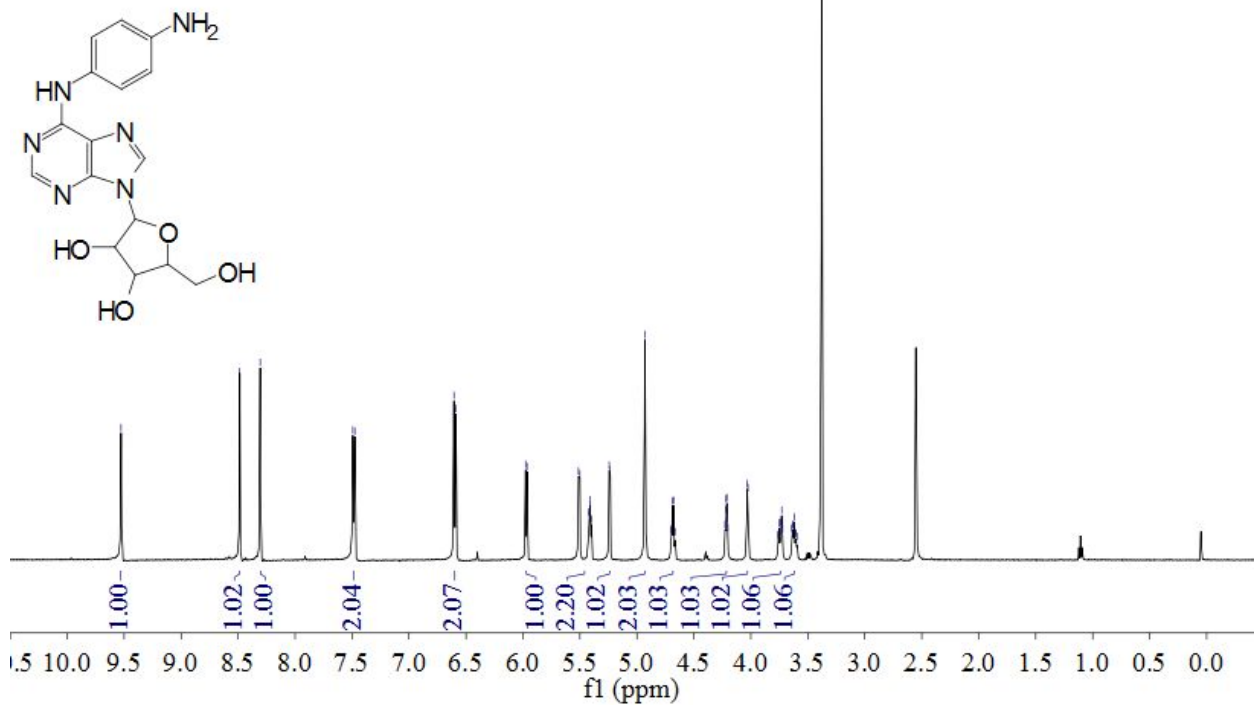

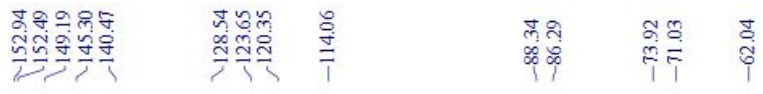
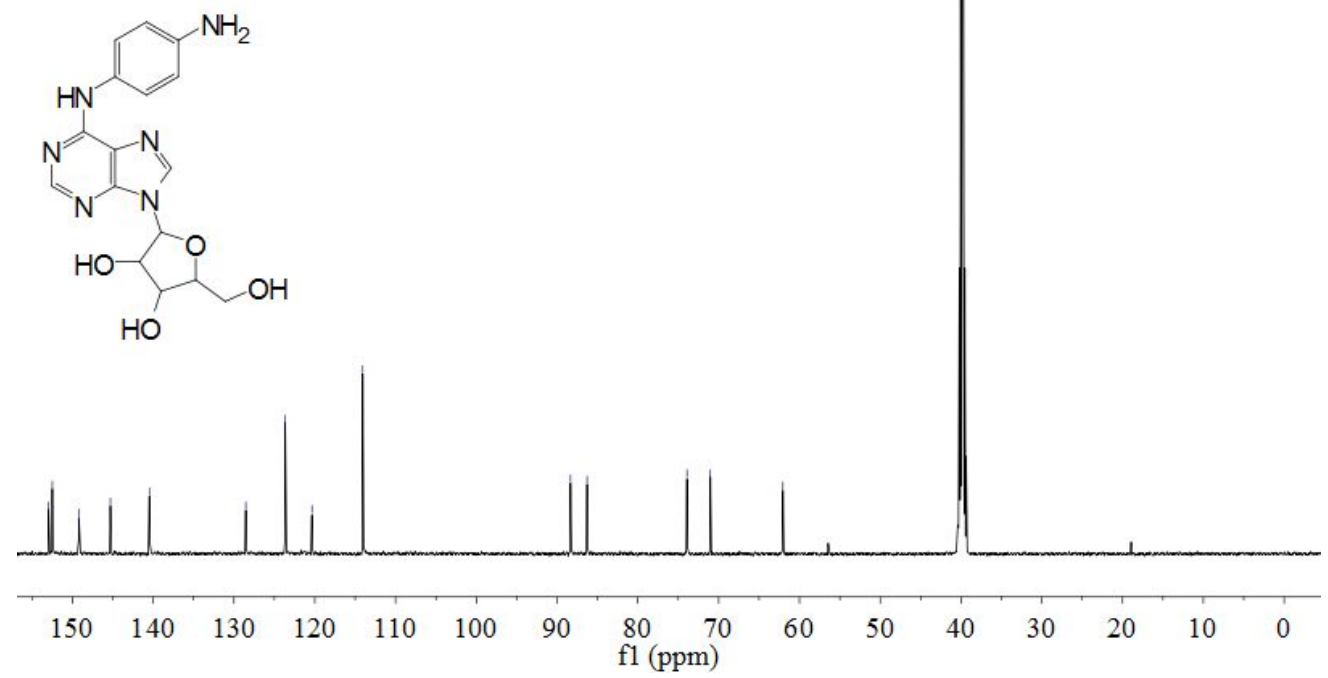

3.2. ${ }^{1} \mathrm{H},{ }^{13} \mathrm{C}$ NMR, HRMS data of title compounds (1-25)

Spectral data for compound 1 


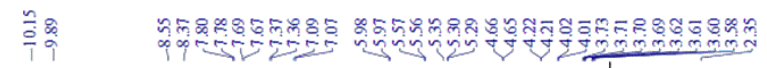
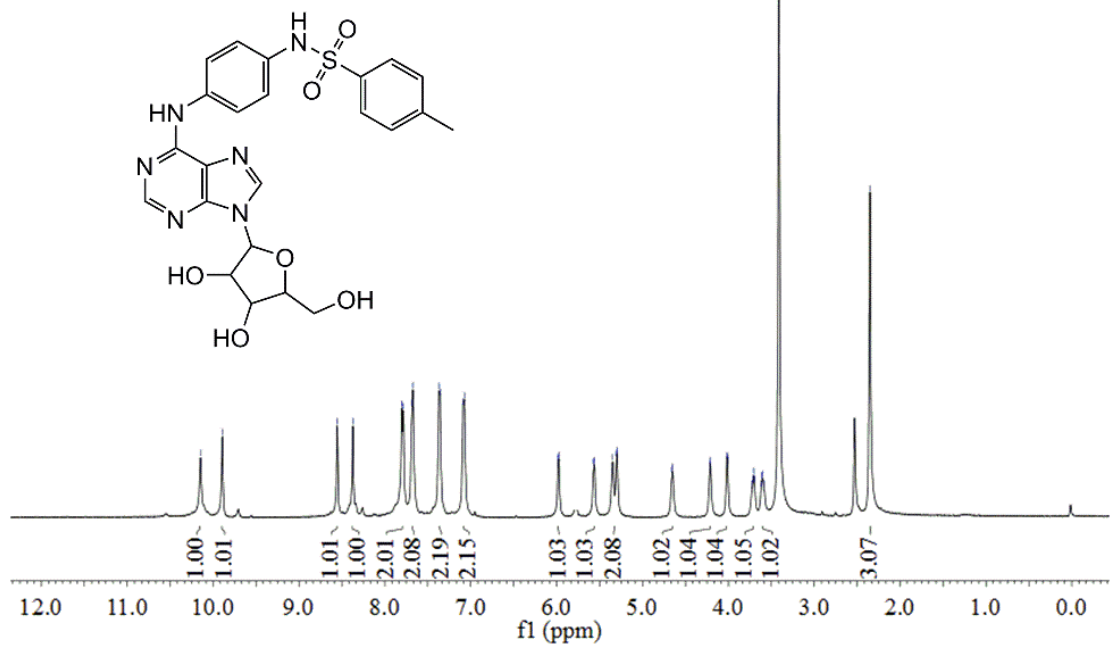

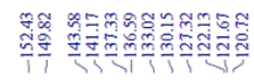

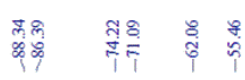

$\stackrel{n}{\uparrow}$

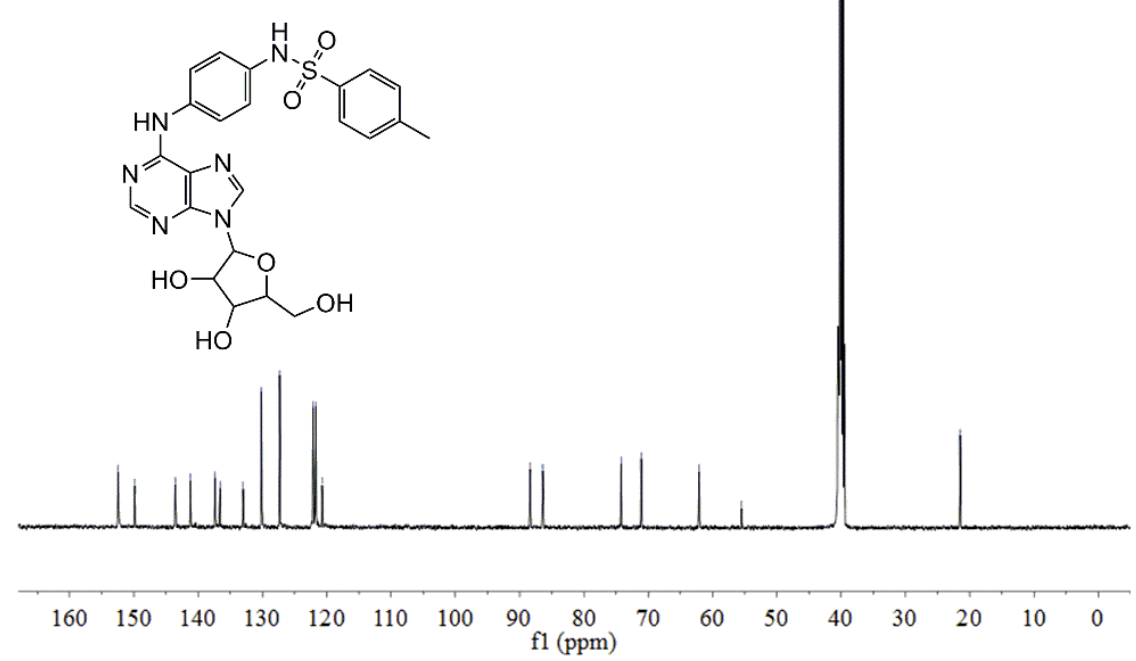

2017091923 \#67 RT: 0.66 AV: 1 NL: $1.87 E 7$
T: FTMS + p ESI Full ms [100.0000-800.0000]

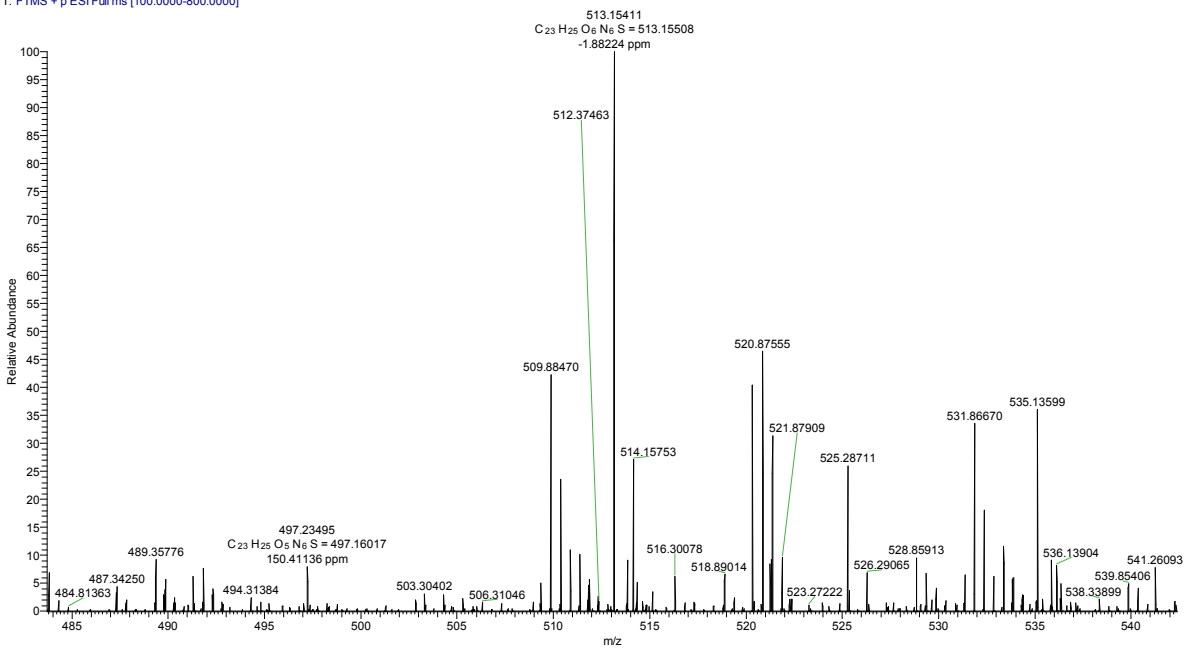

S-18 


\section{Spectral data for compound 2}

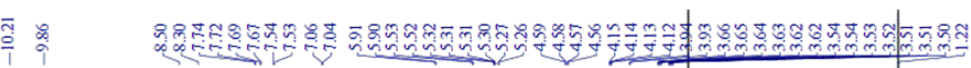

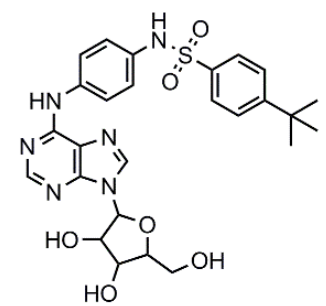

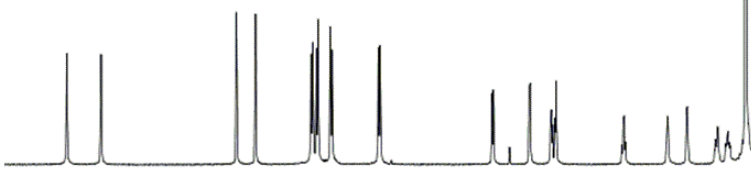

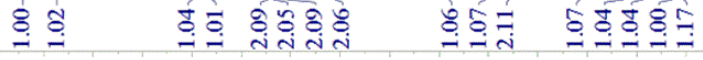

$\stackrel{\infty}{\circ}$

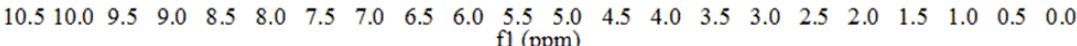

สิ่

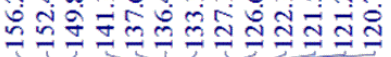

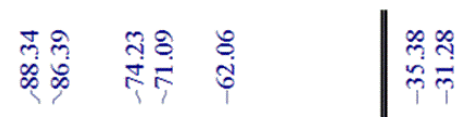

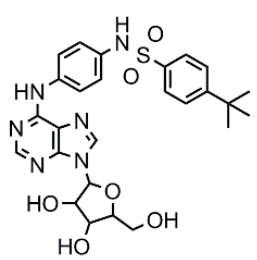
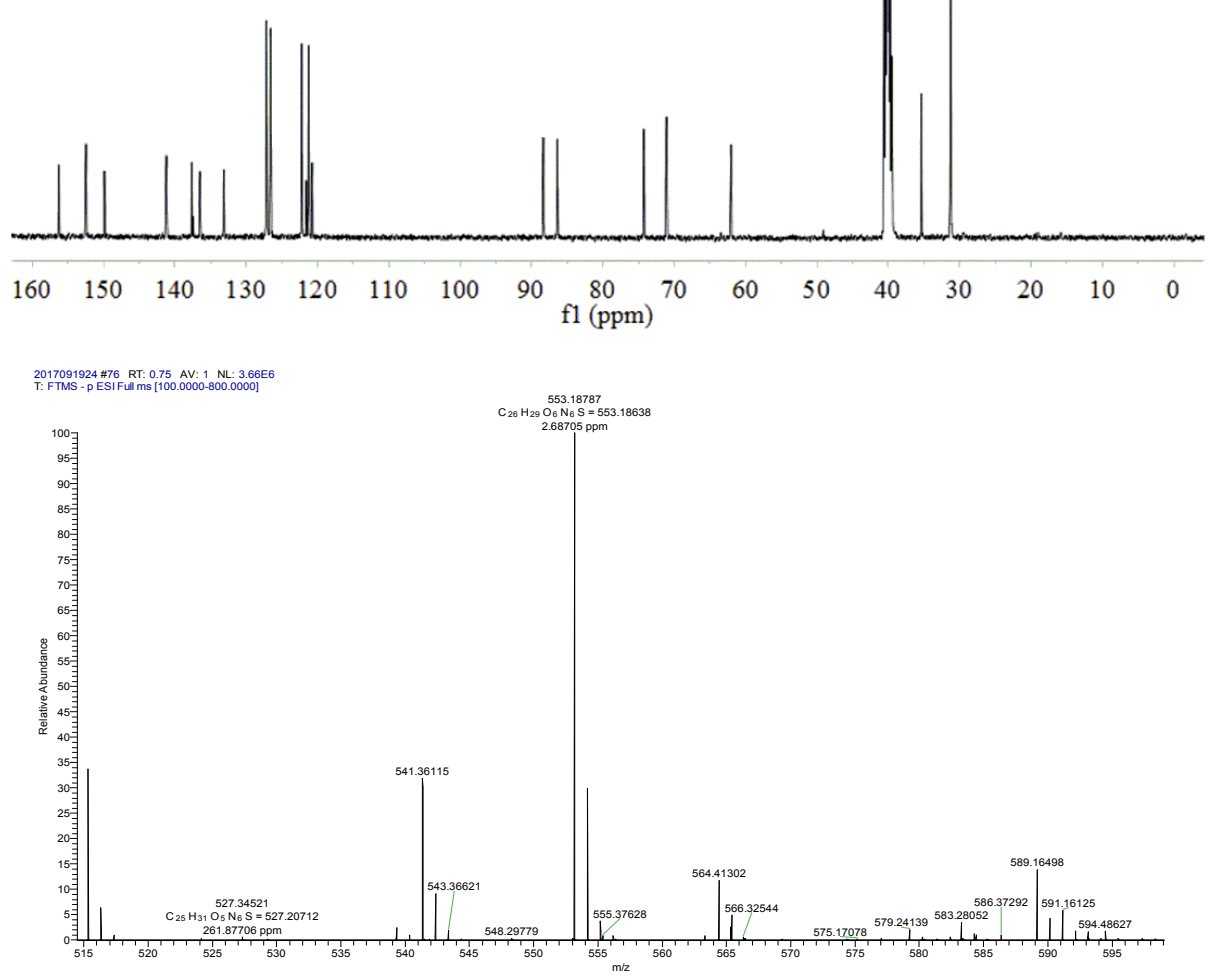

S-19 


\section{Spectral data for compound 3}

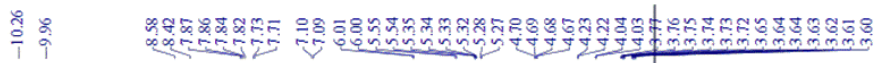

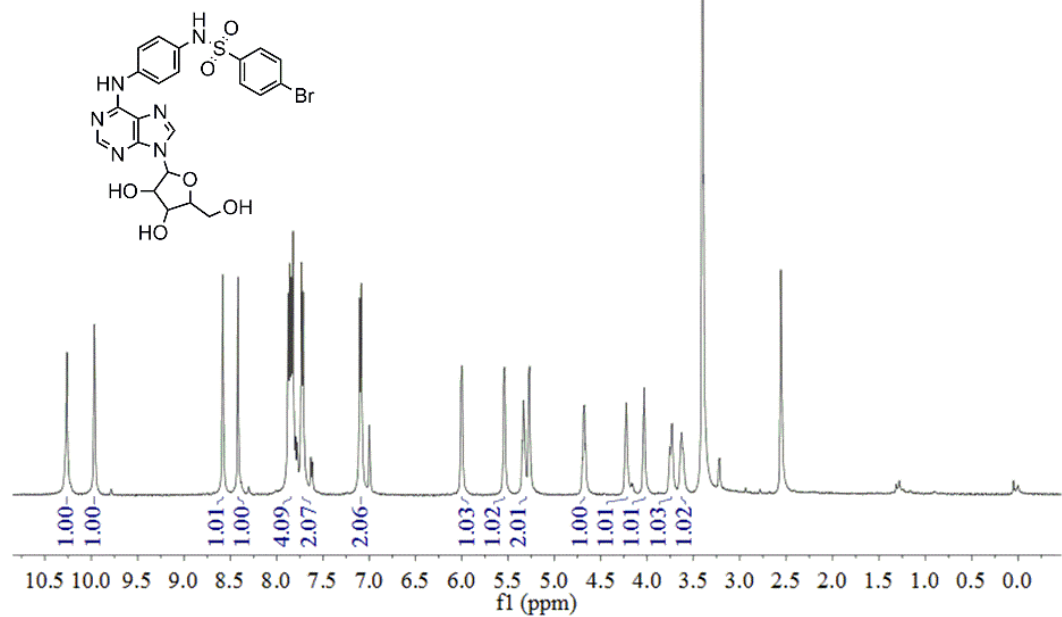

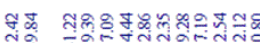

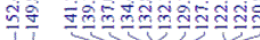

f1 (ppm)
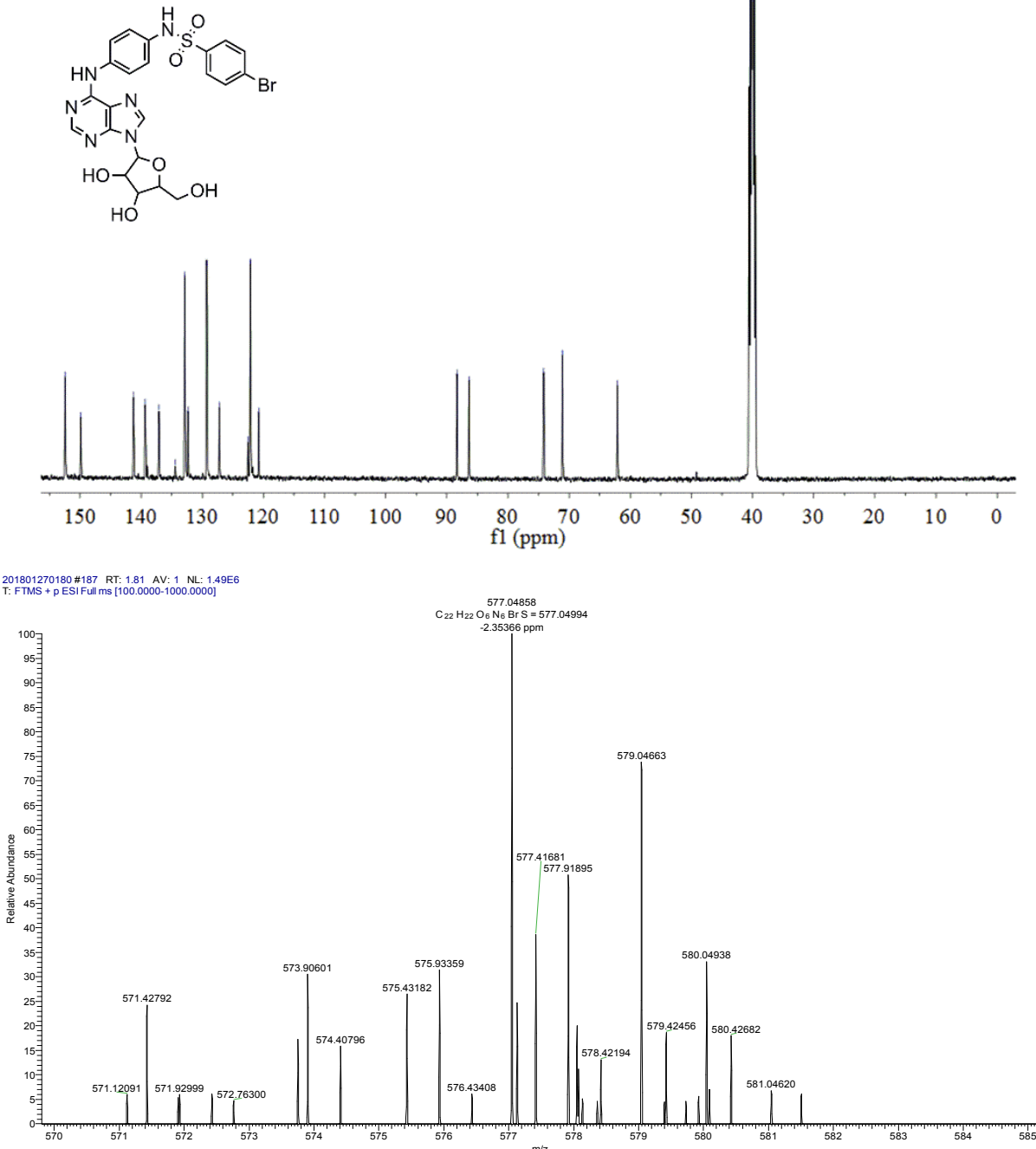


\section{Spectral data for compound 4}

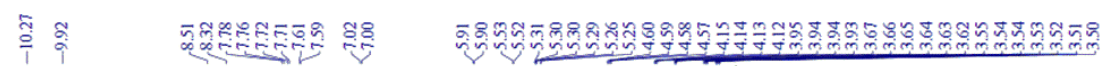

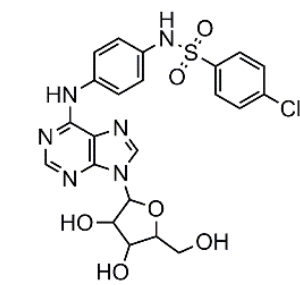

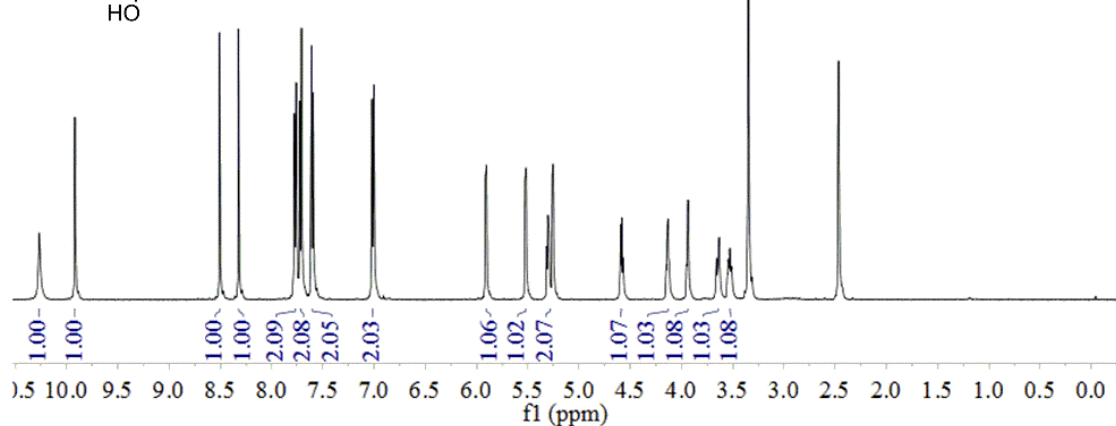

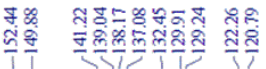

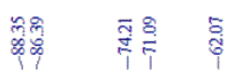<smiles>OCC1OC(n2cnc3c(Nc4ccc(N[SH](O)(O)c5ccc(Cl)cc5)cc4)ncnc32)C(O)C1O</smiles>
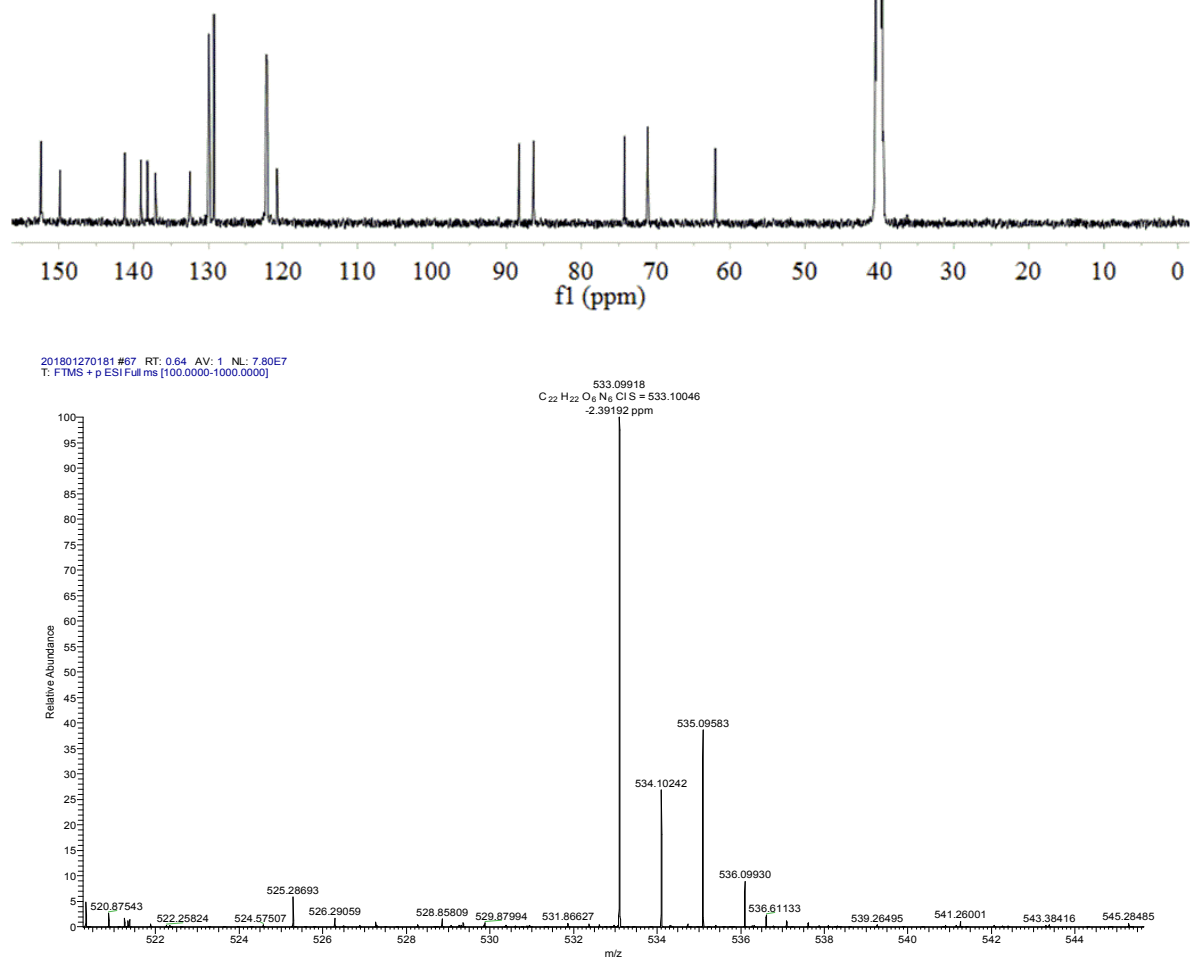


\section{Spectral data for compound 5}

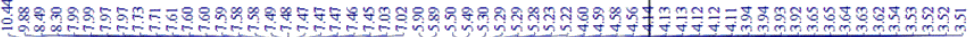
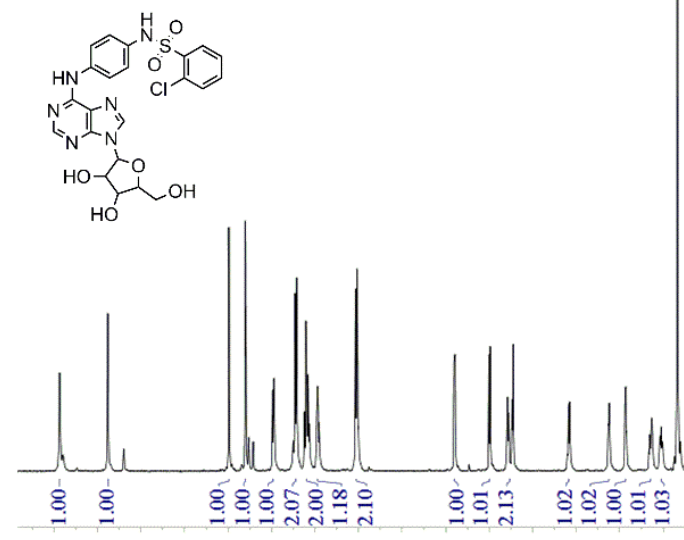

$\begin{array}{llllllllllllllllllllll}10.5 & 10.0 & 9.5 & 9.0 & 8.5 & 8.0 & 7.5 & 7.0 & 6.5 & 6.0 & 5.5 & 5.0 & 4.5 & 4.0 & 3.5 & 3.0 & 2.5 & 2.0 & 1.5 & 1.0 & 0.5 & 0.0\end{array}$ fl (ppm)
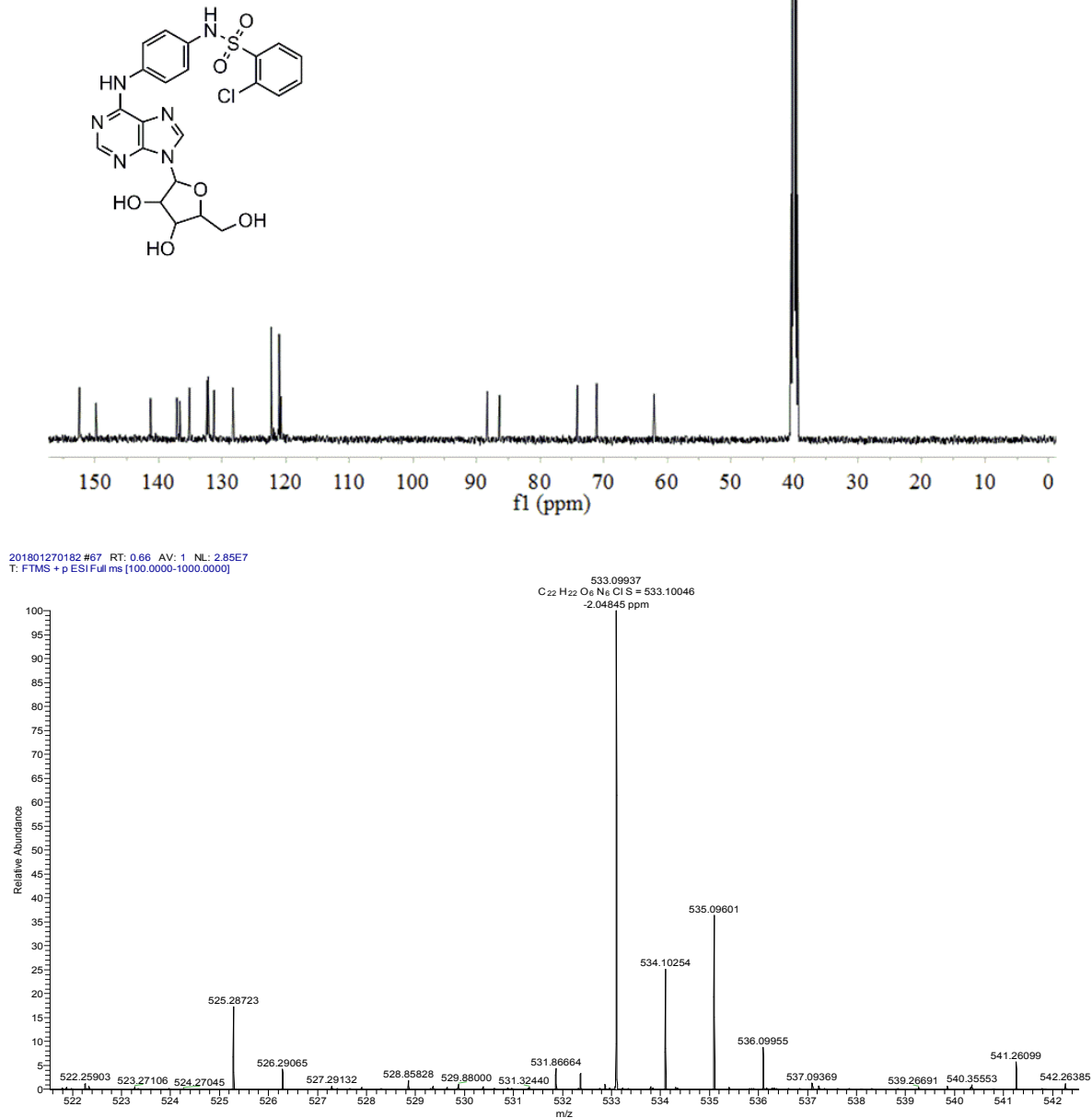


\section{Spectral data for compound 6}

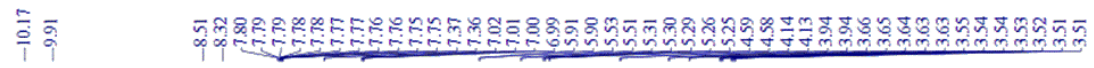

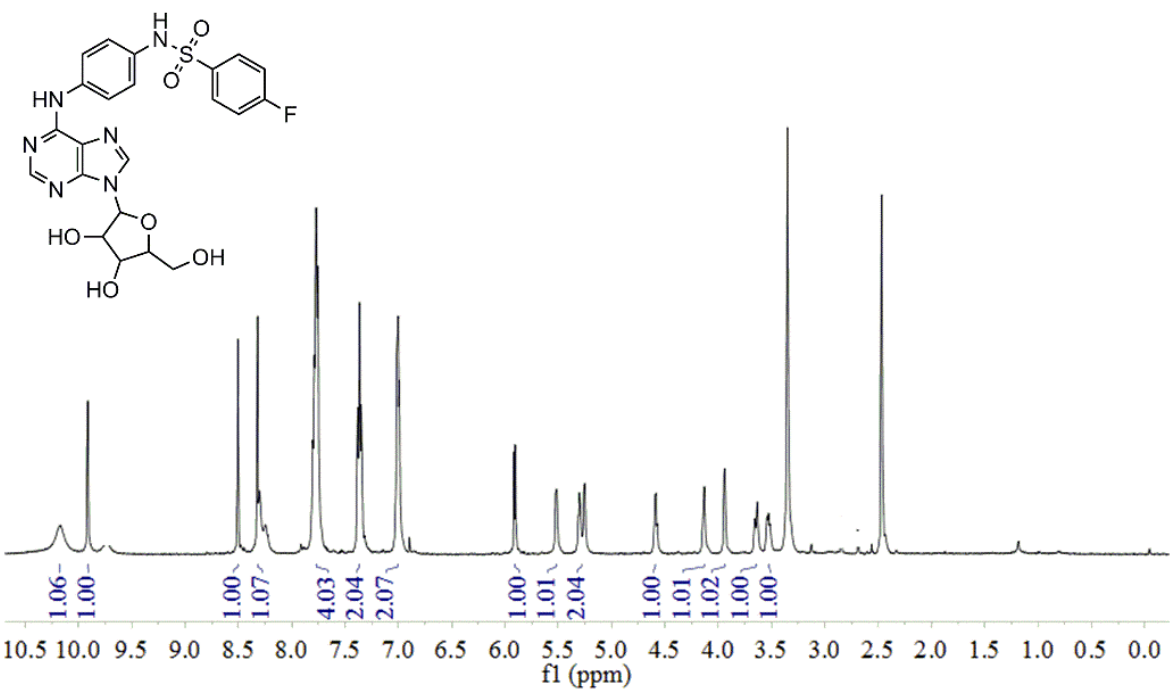

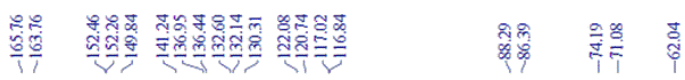
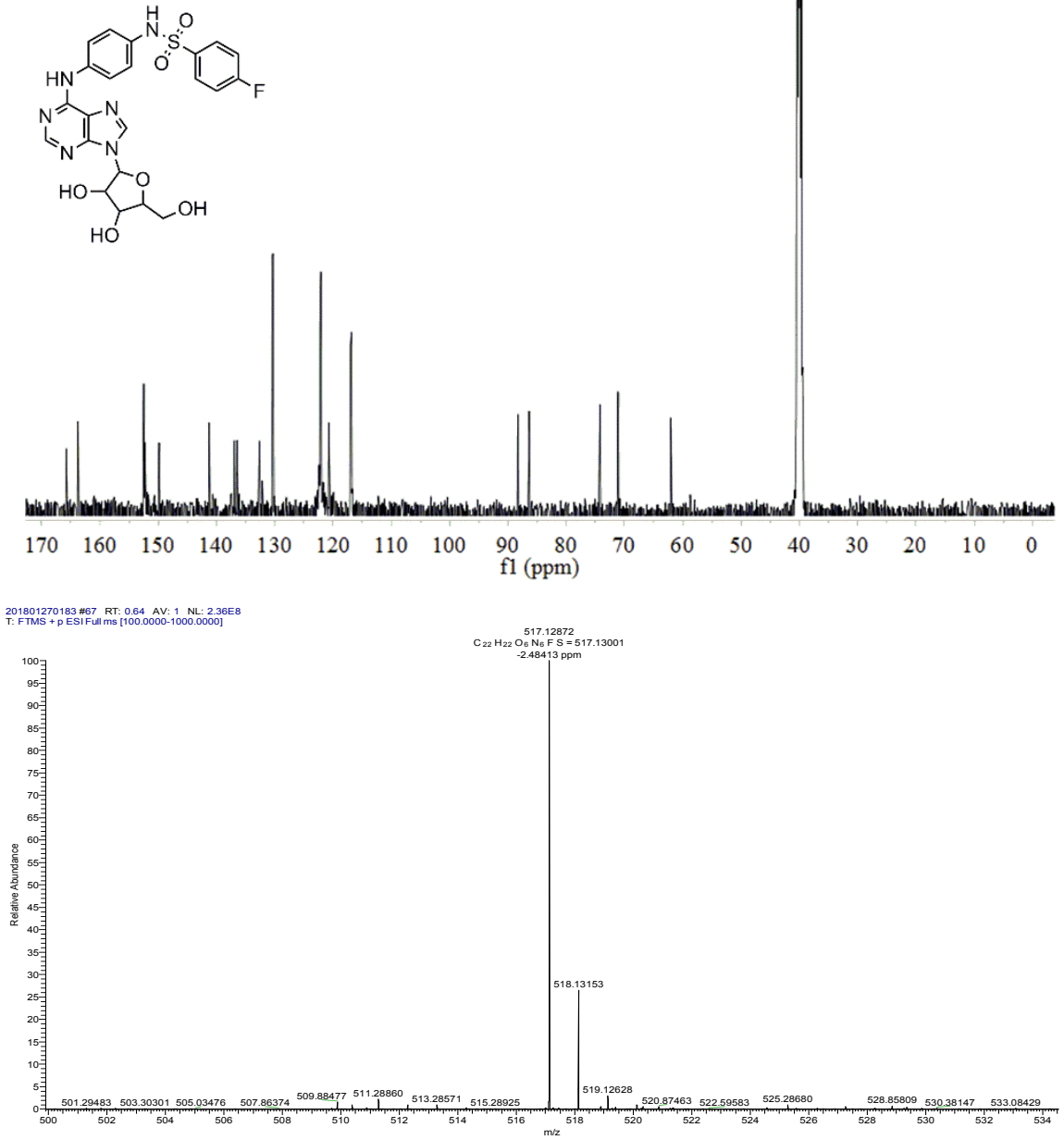


\section{Spectral data for compound 7}

o
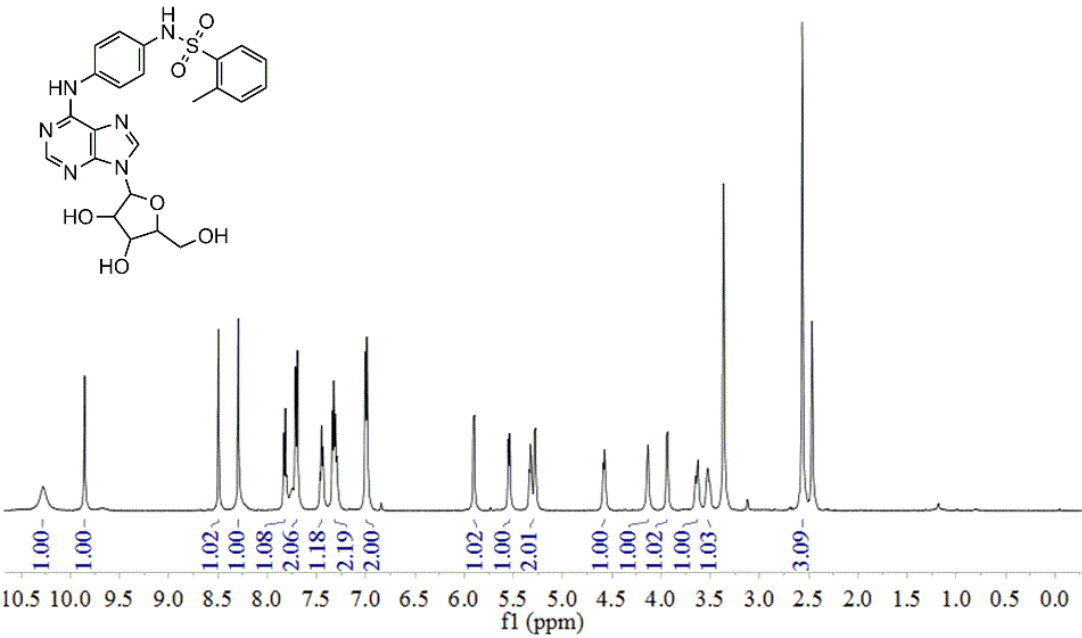

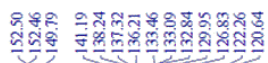

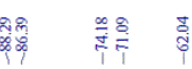
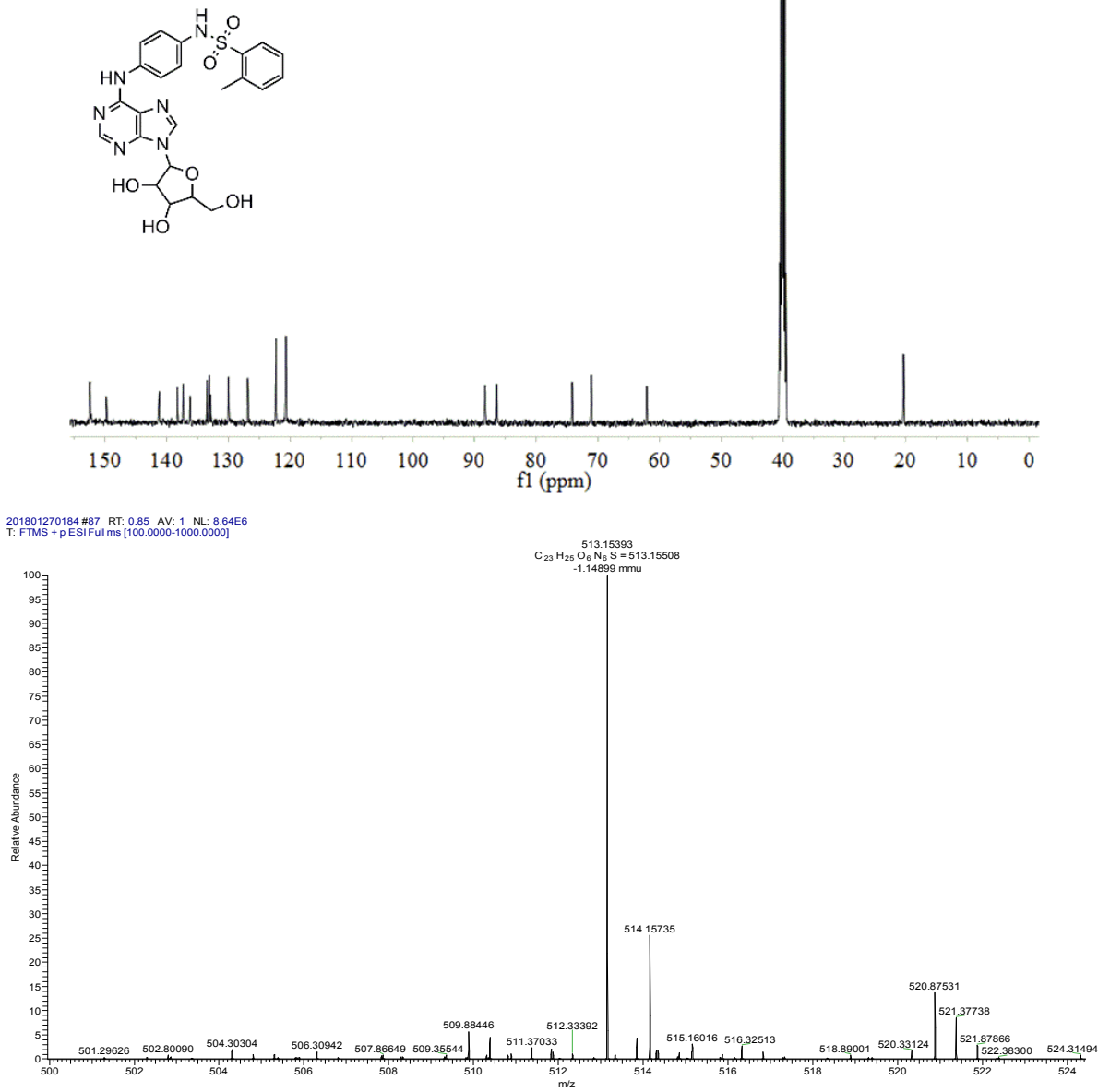


\section{Spectral data for compound 8}

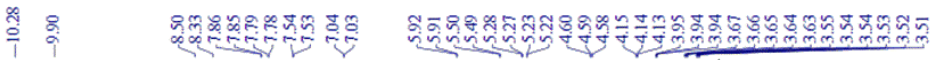
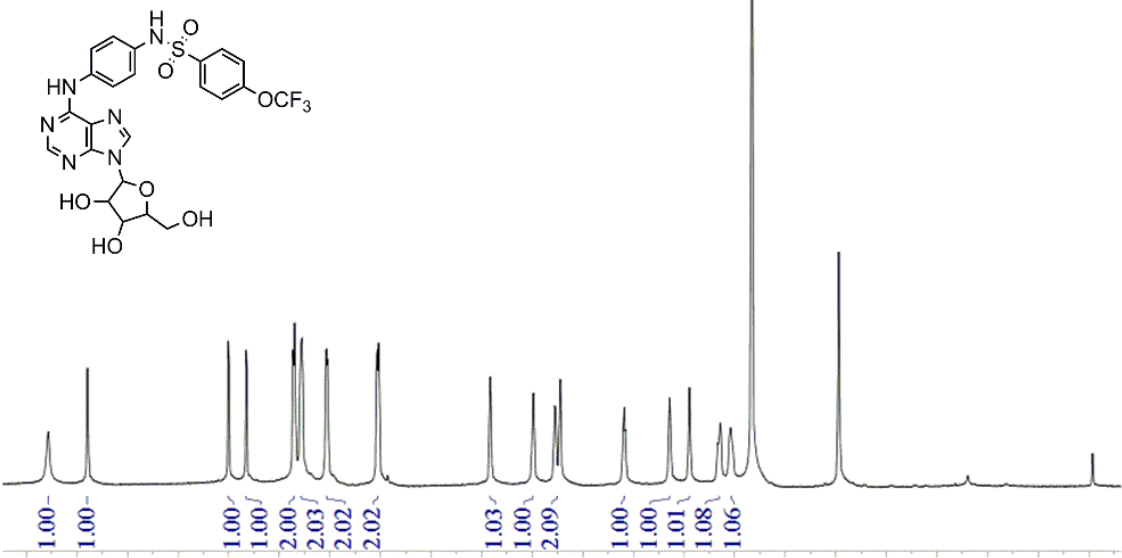

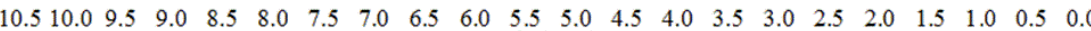
f1 (ppm)

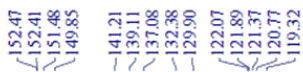
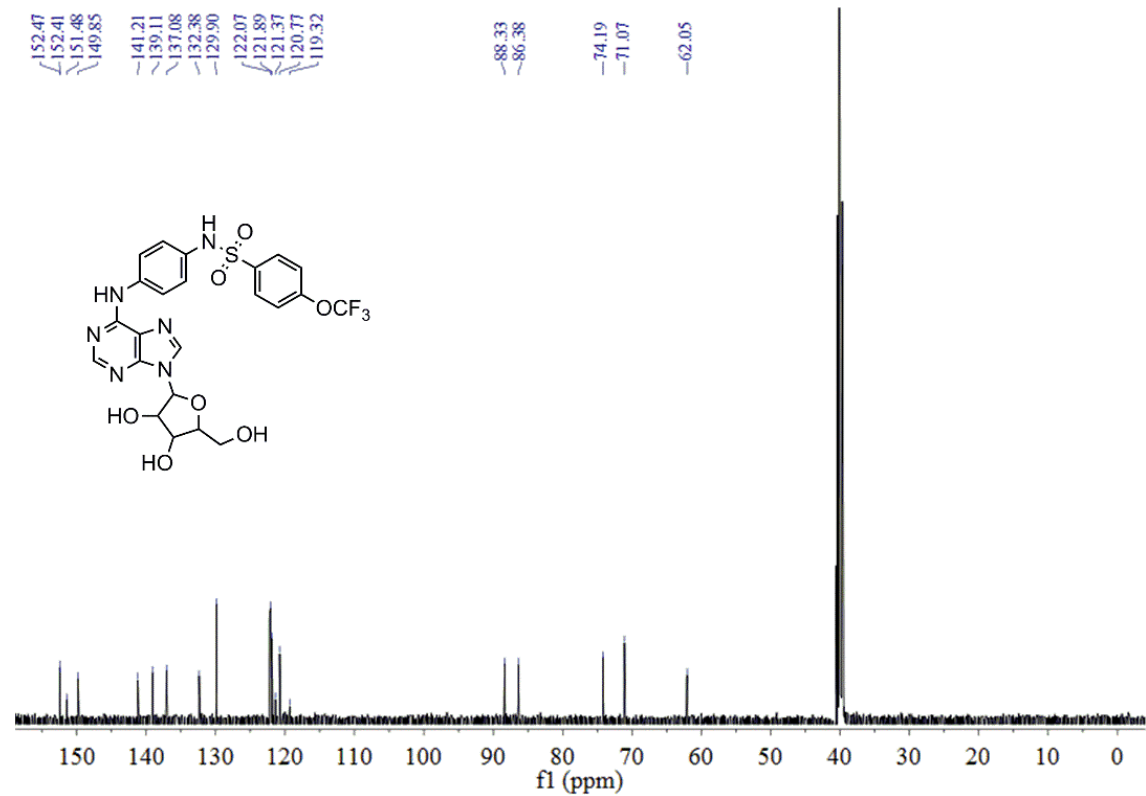

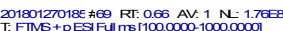

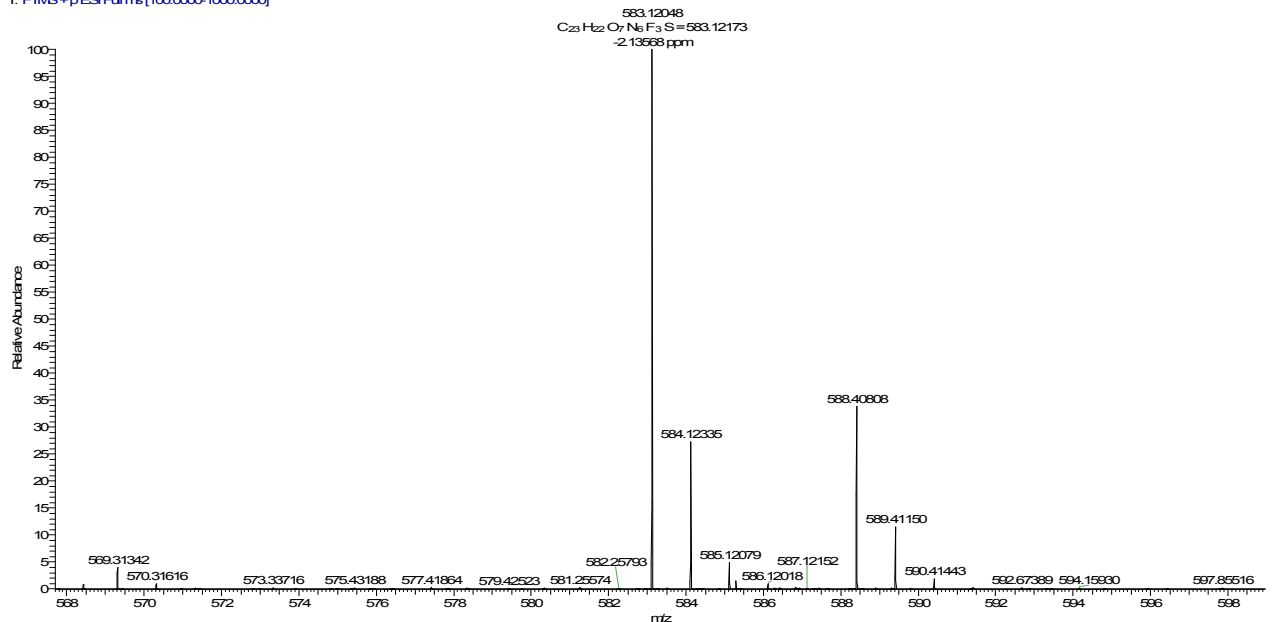

Spectral data for compound 9 

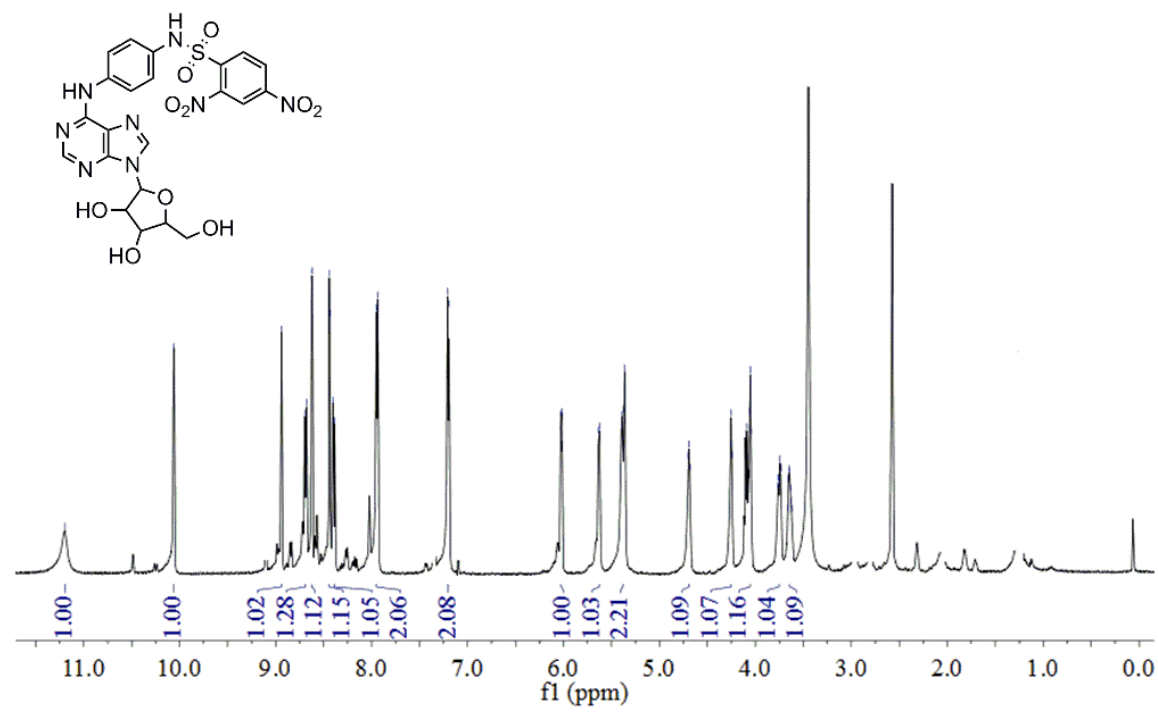

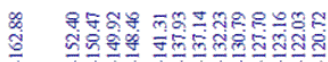
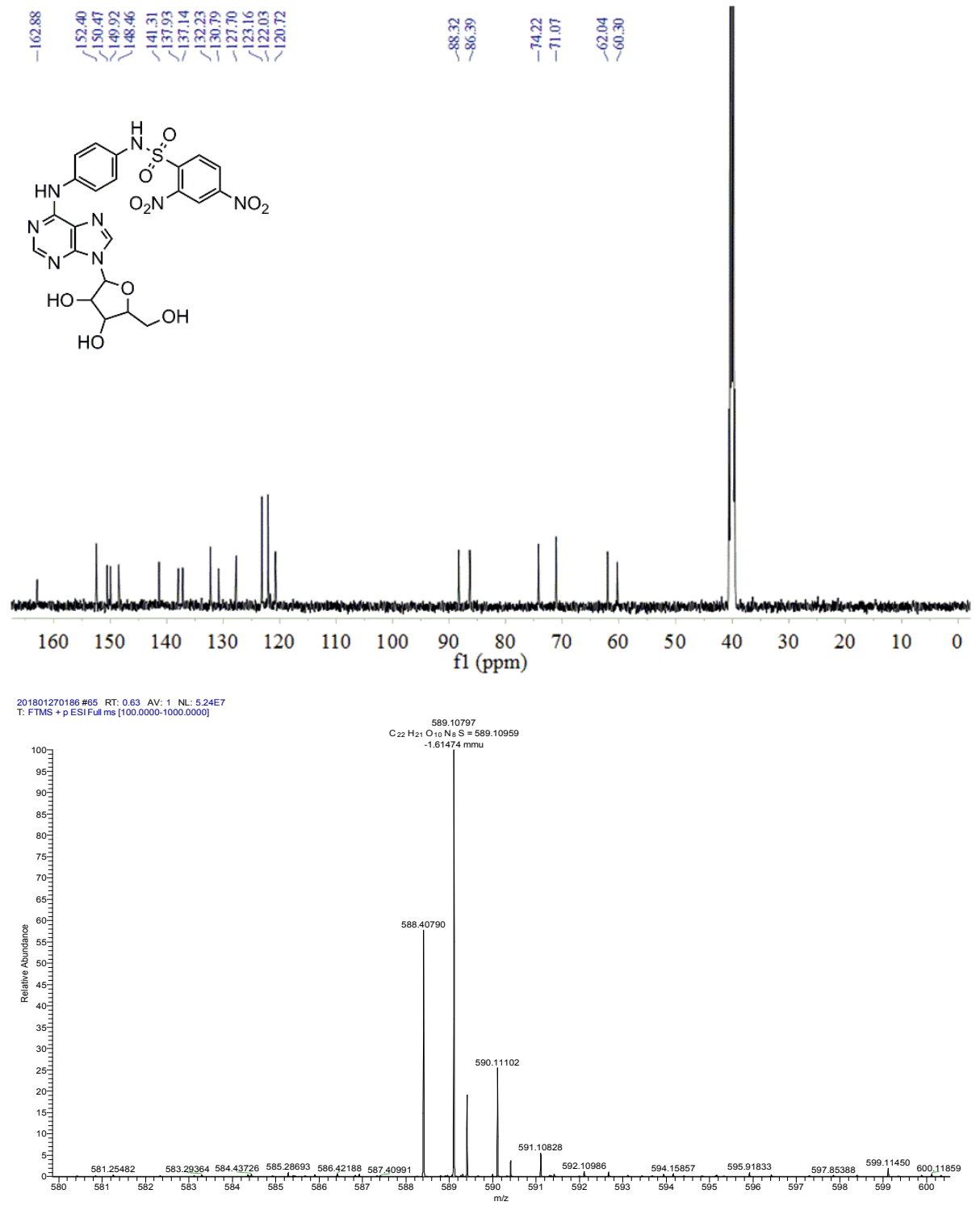

\section{Spectral data for compound 10}



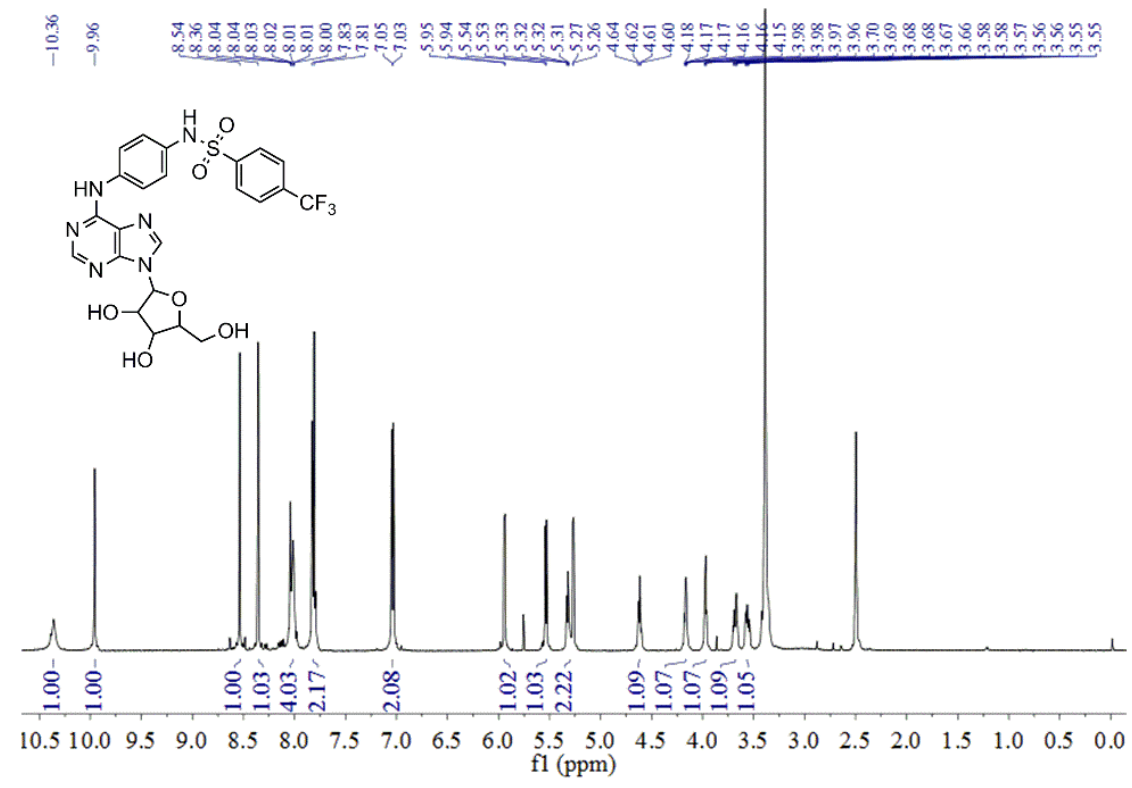

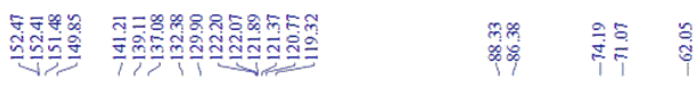
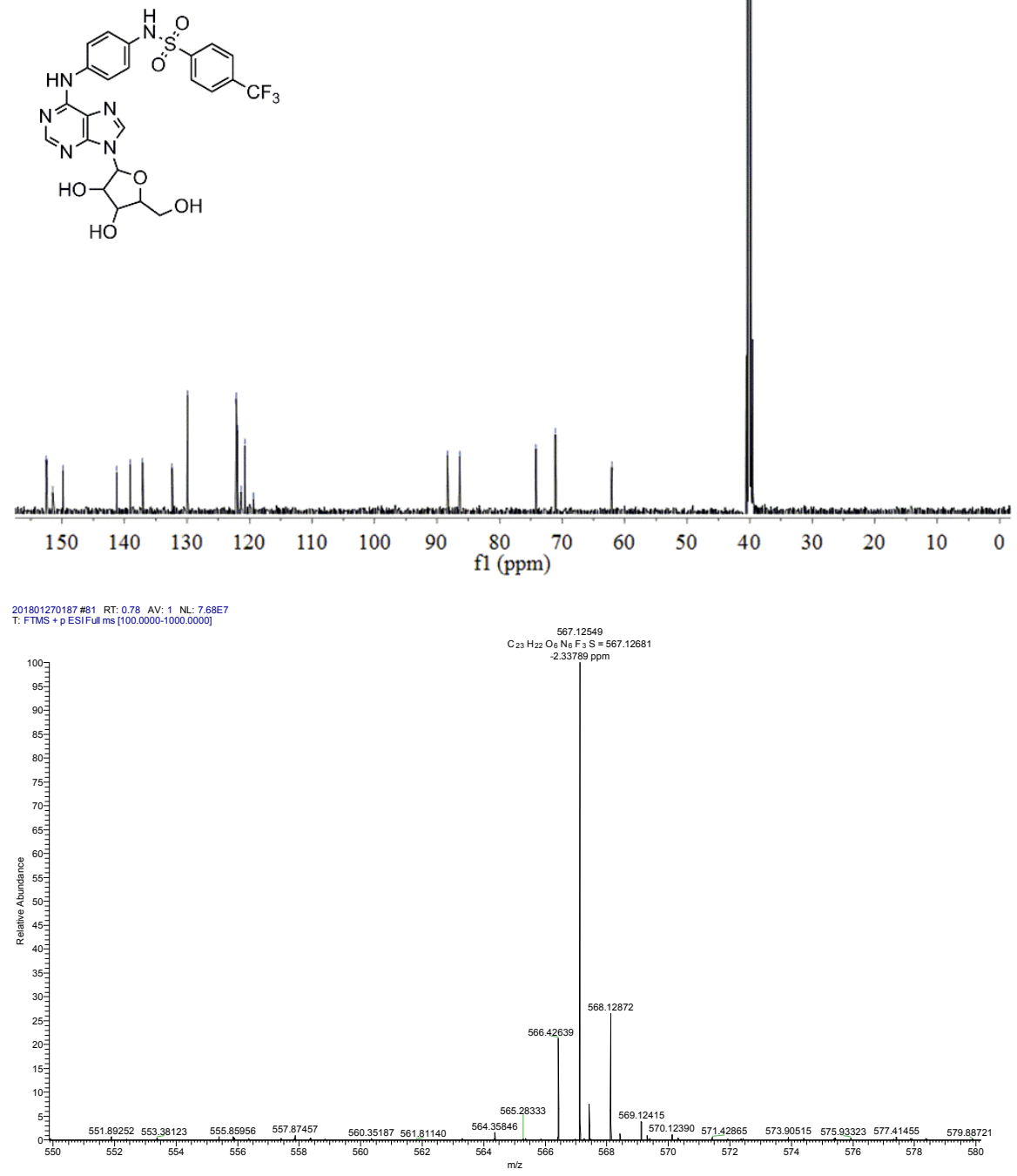

\section{Spectral data for compound 11}



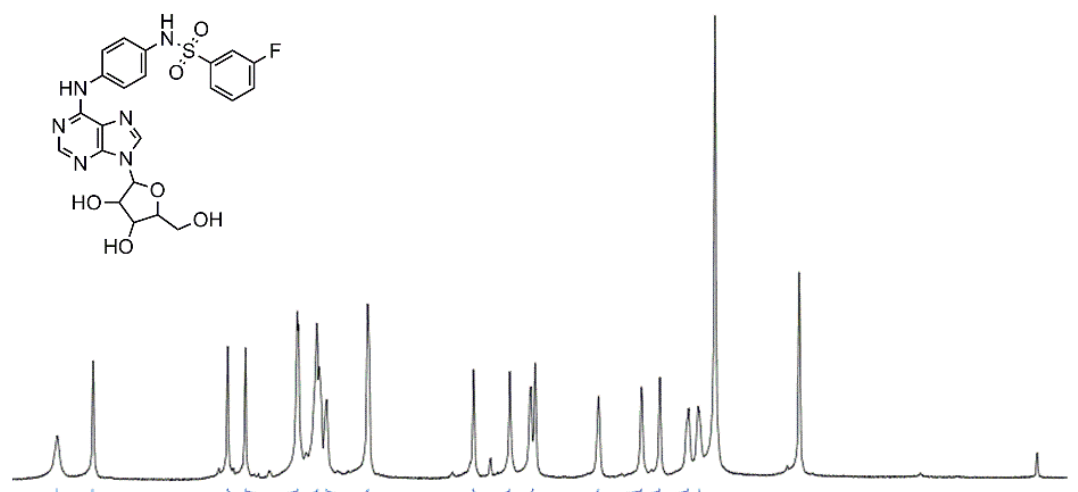

8

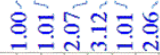

$8 \%$ 508

$\begin{array}{llllllllllllllllllllll}10.5 & 10.0 & 9.5 & 9.0 & 8.5 & 8.0 & 7.5 & 7.0 & 6.5 & 6.0 & 5.5 & 5.0 & 4.5 & 4.0 & 3.5 & 3.0 & 2.5 & 2.0 & 1.5 & 1.0 & 0.5 & 0.0\end{array}$ fi (ppm)

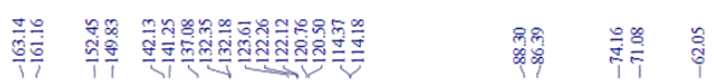<smiles>O=S(=O)(Nc1ccc(Nc2ncnc3c2ncn3C2OC(CO)C(O)C2O)cc1)c1cccc(F)c1</smiles>

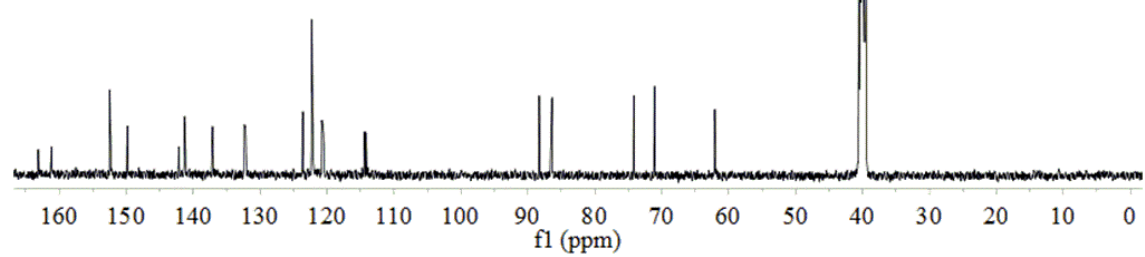

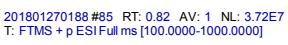

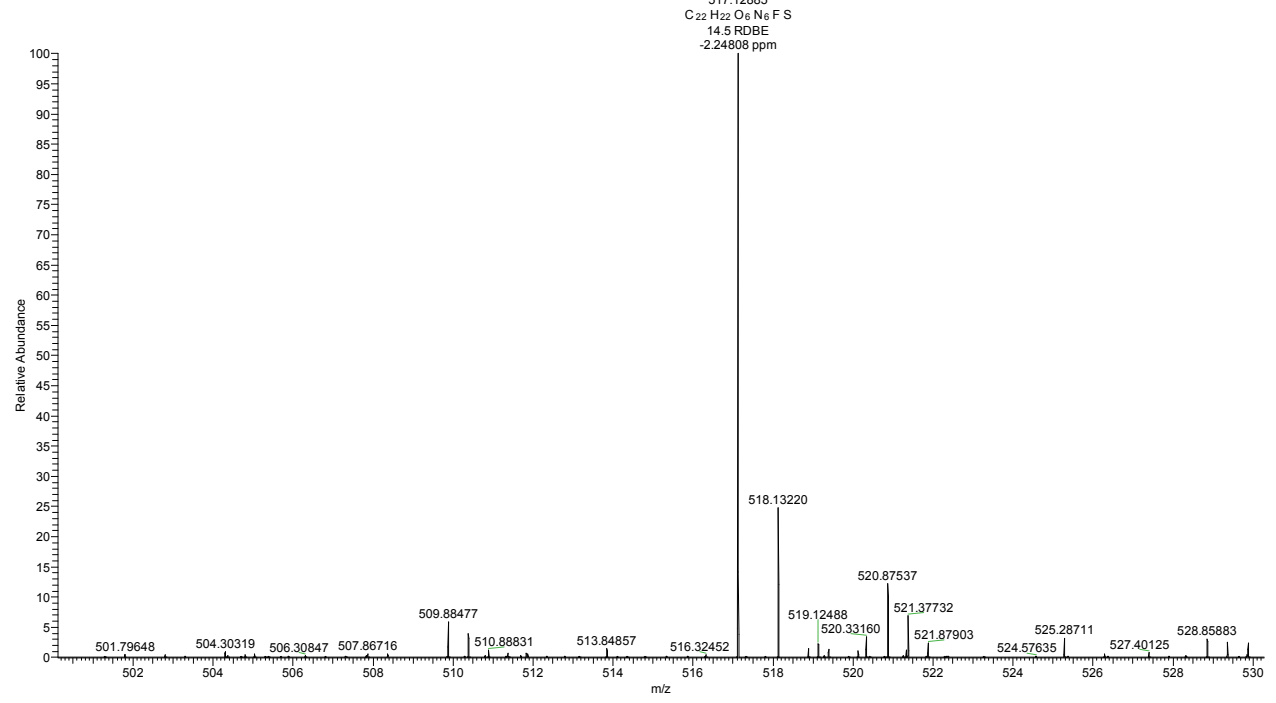




\section{Spectral data for compound 12}

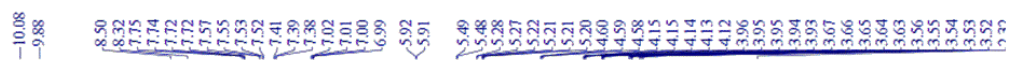
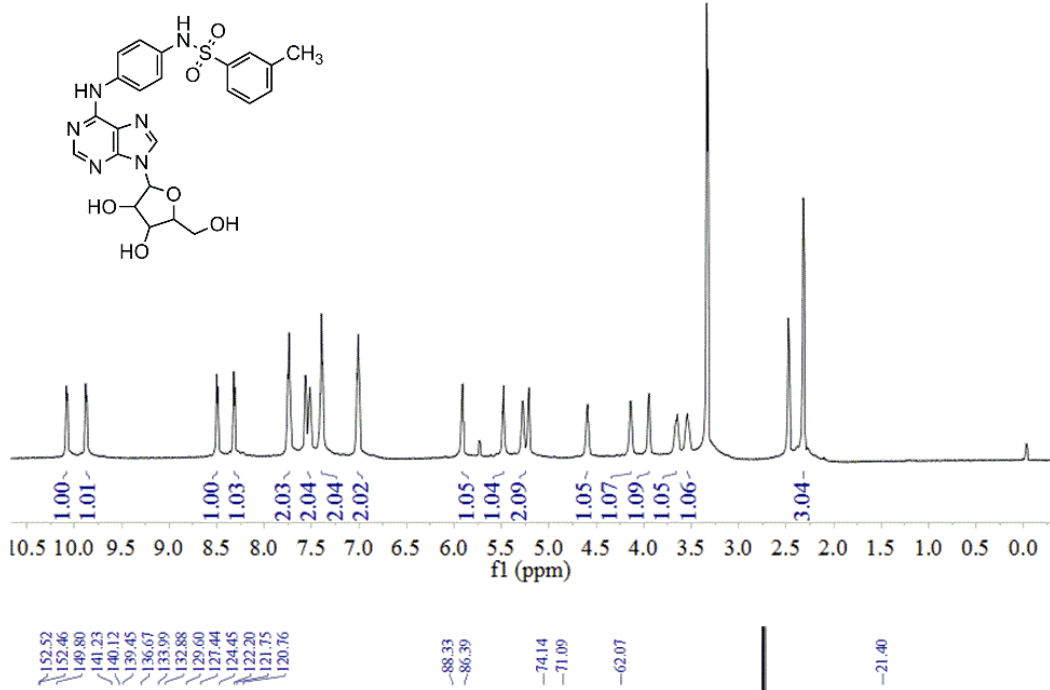

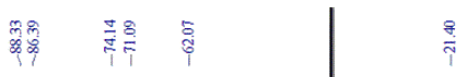
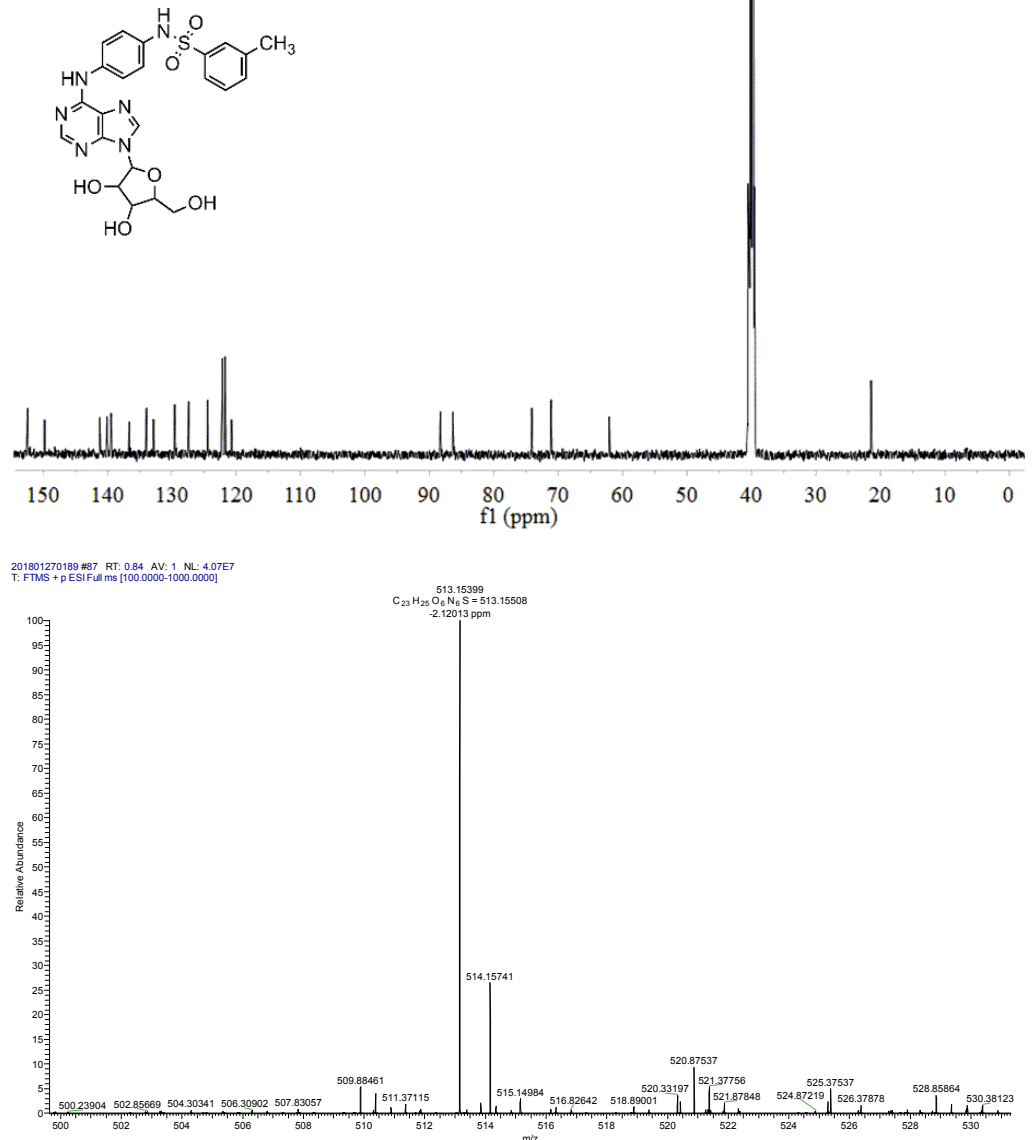


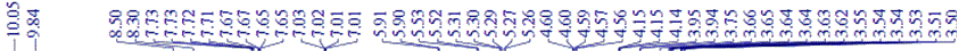

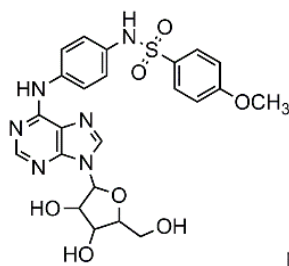

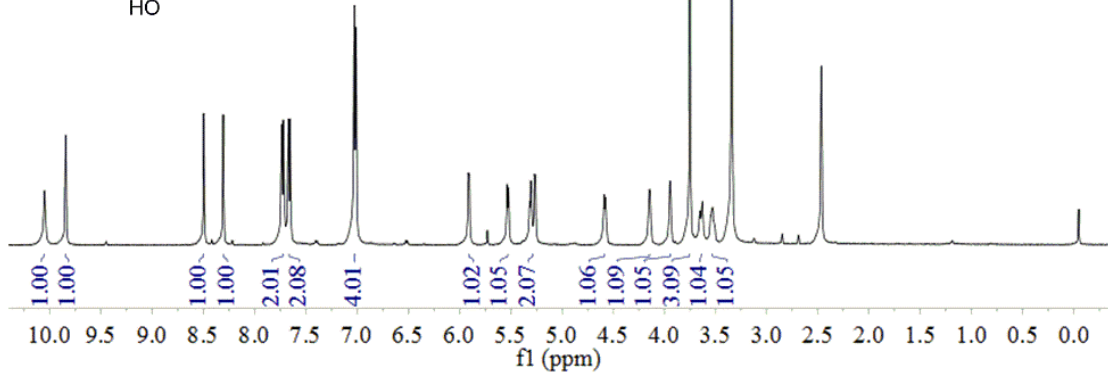

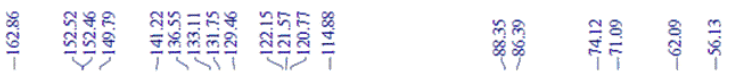

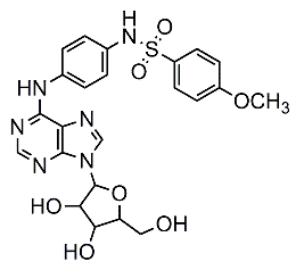

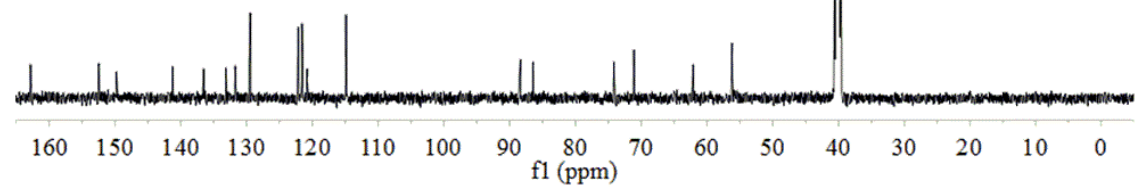

201801270190 \#85 RT: 0.83 AV: 1 NL: 1.6967
T: FTMS + p ESIFull ms $[100.0000-1000.0000]$

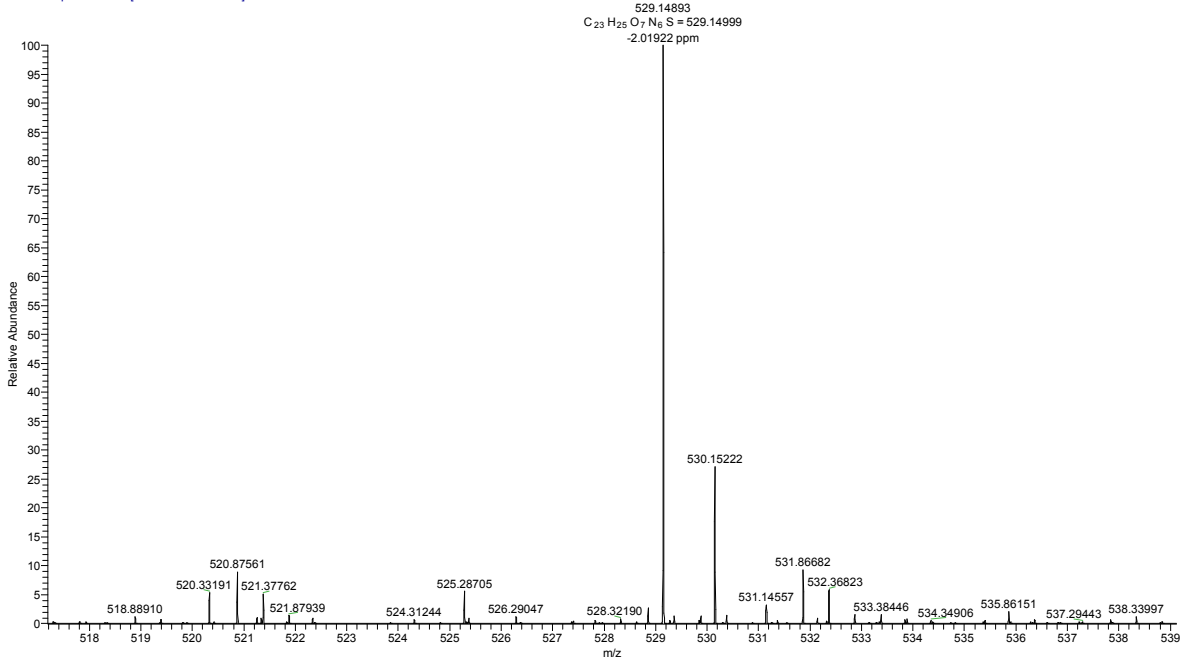

Spectral data for compound 14 


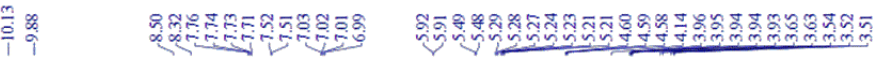

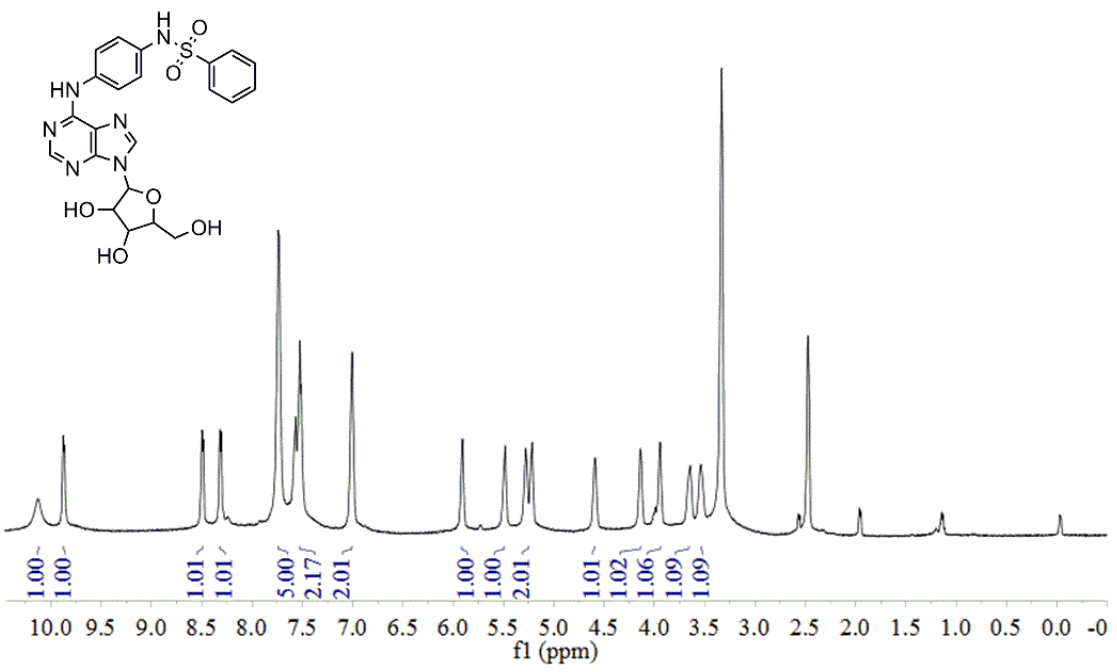

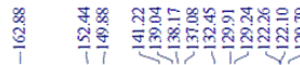

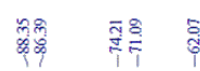
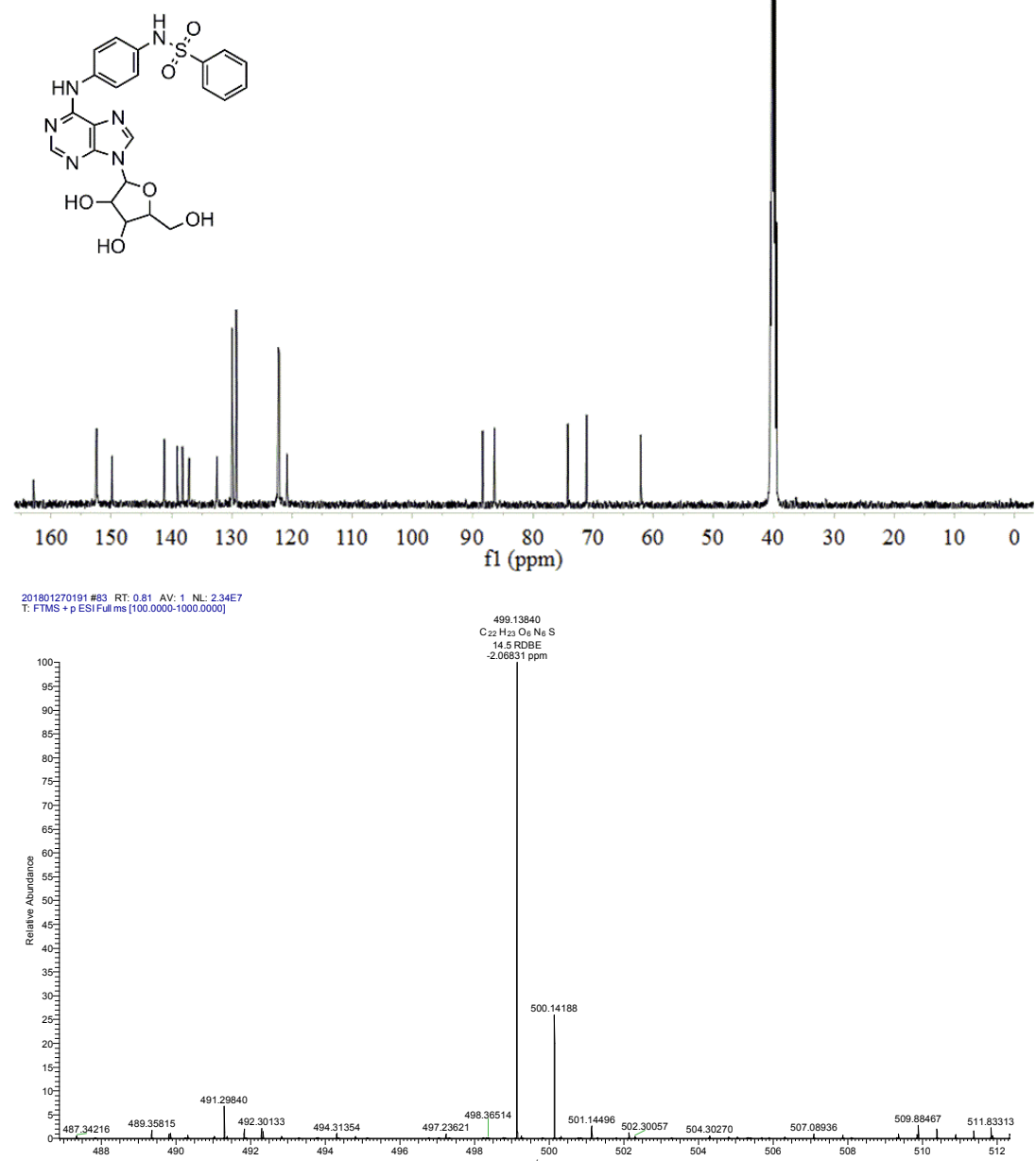

\section{Spectral data for compound 15}




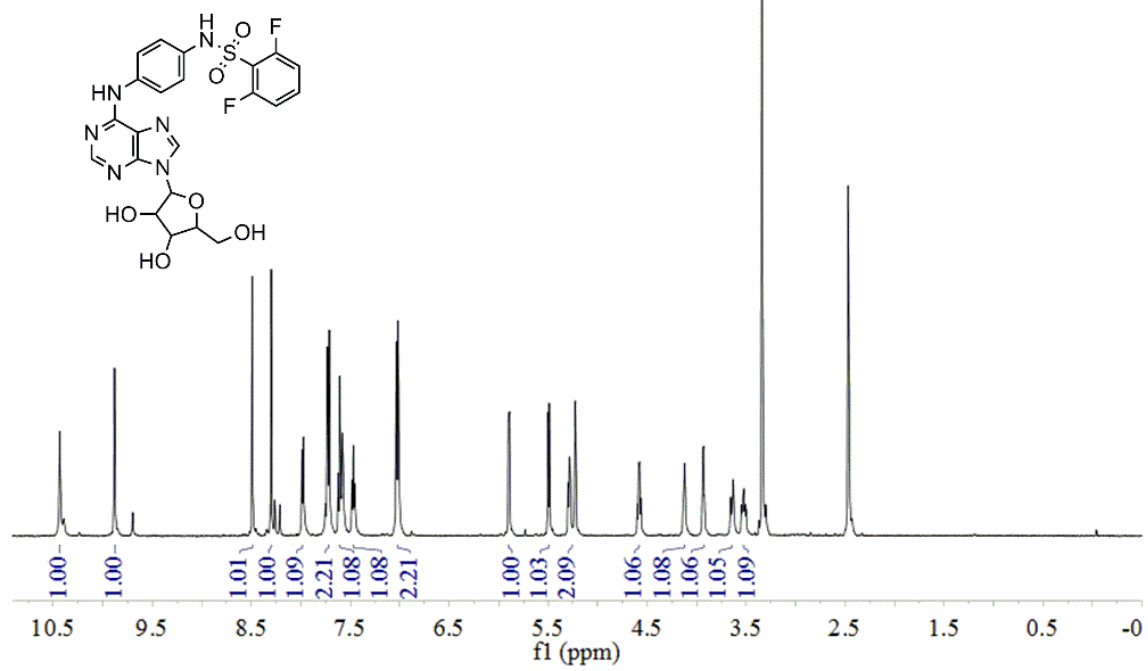

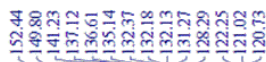

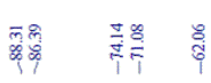
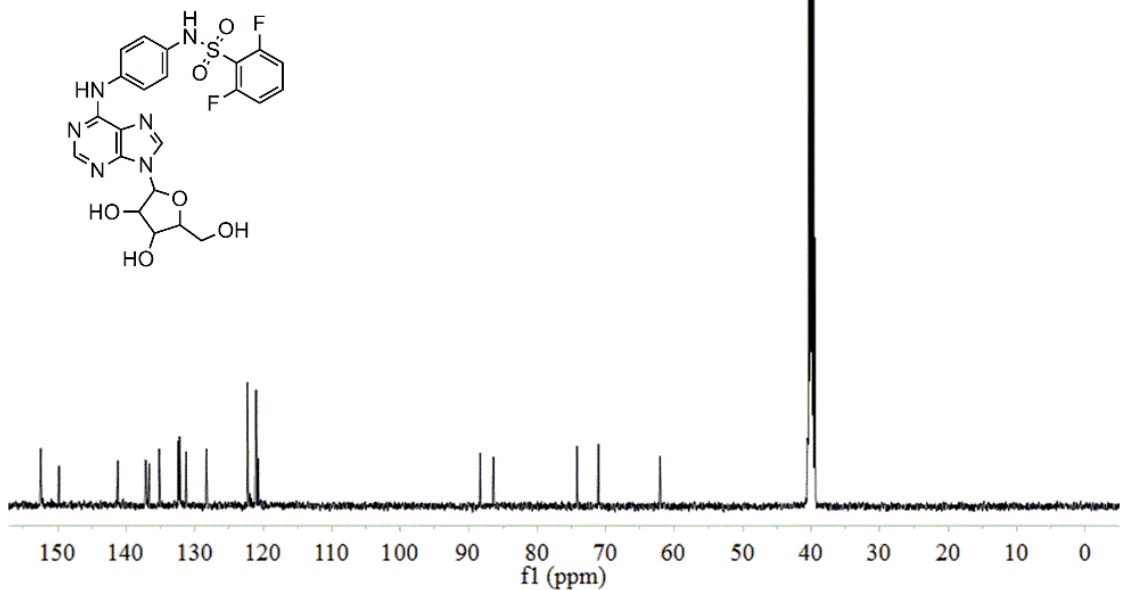

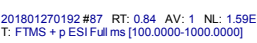

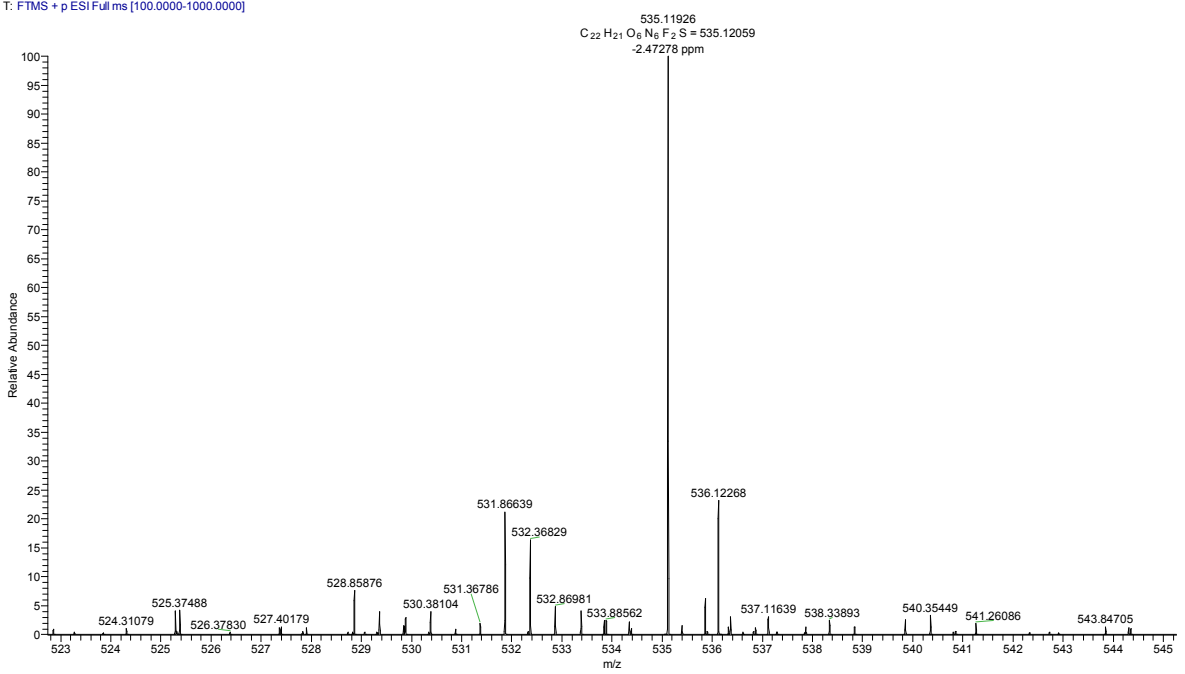

Spectral data for compound 16 
茟

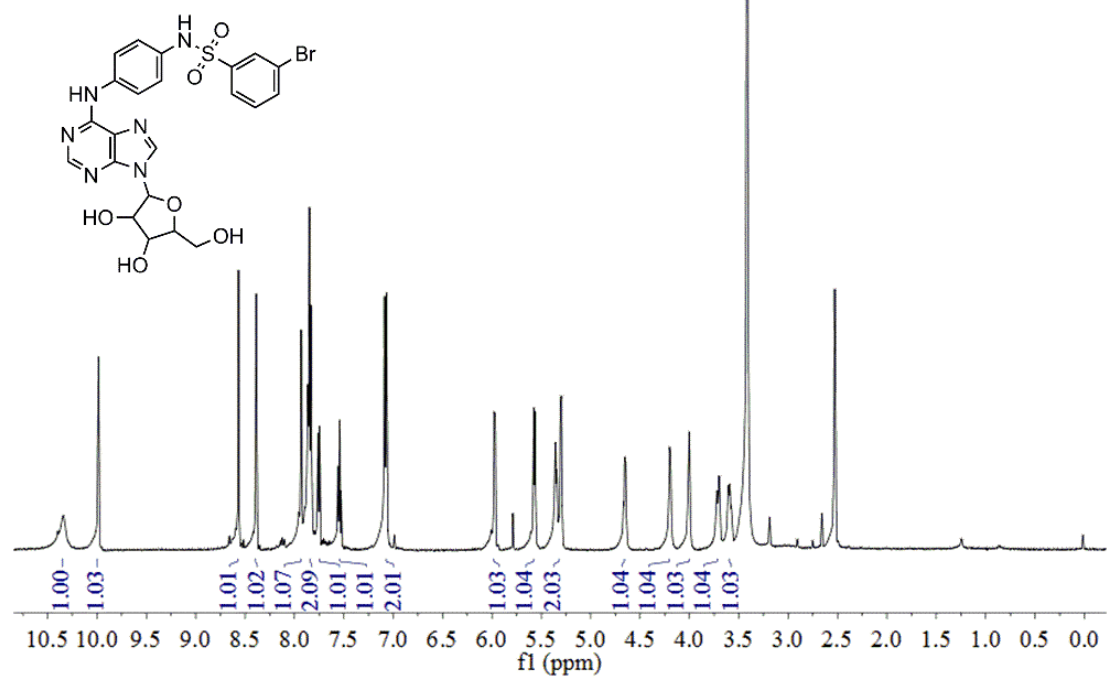

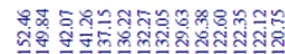

离舫<smiles>OCC(O)C(O)C(O)N1CNC2NCN(c3ccc(Nc4cccc(Br)c4)cc3)CC21</smiles>
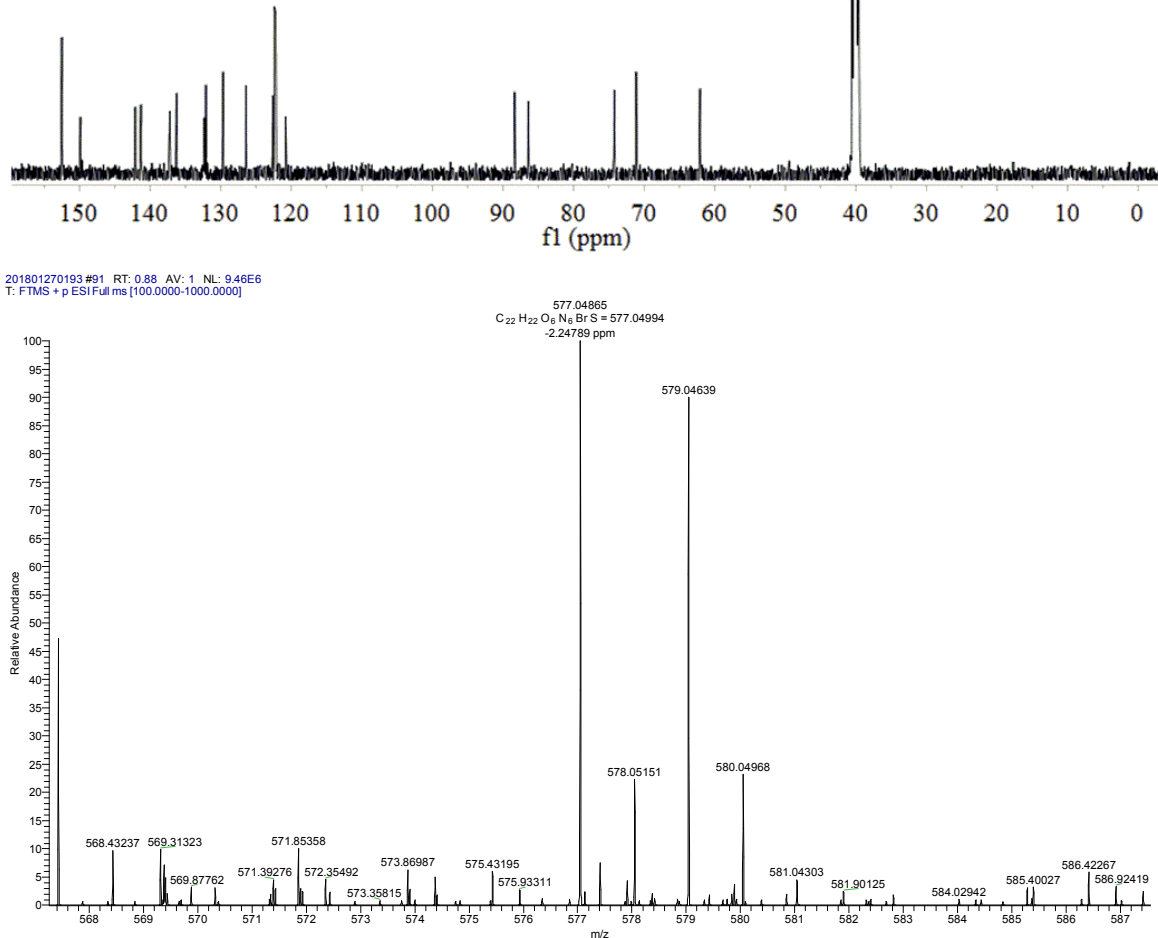

\section{Spectral data for compound 17}



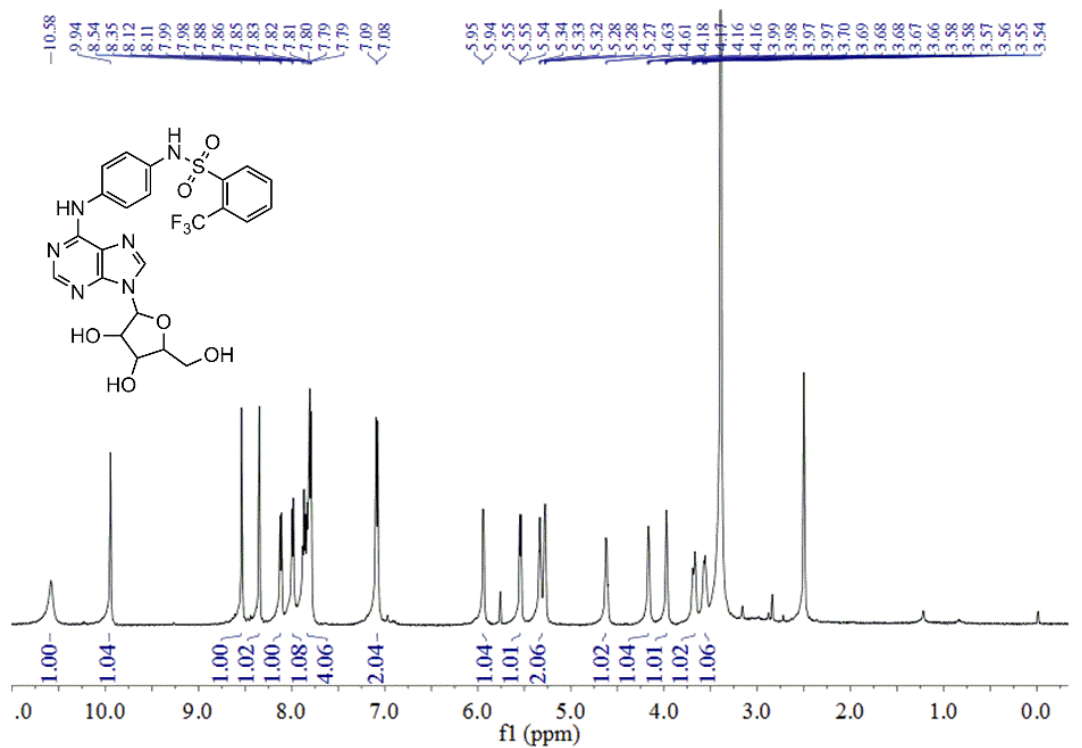

尔营
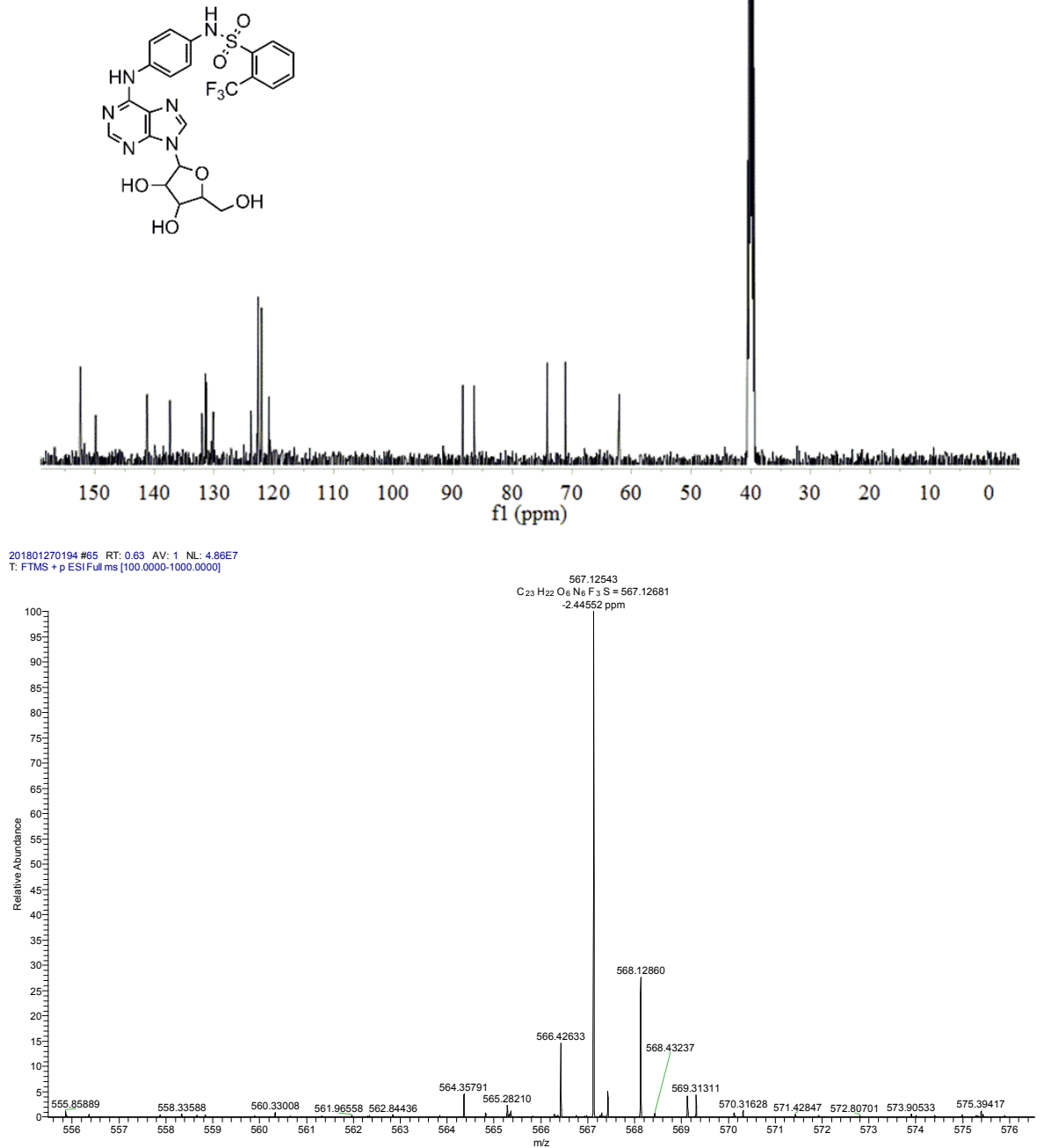


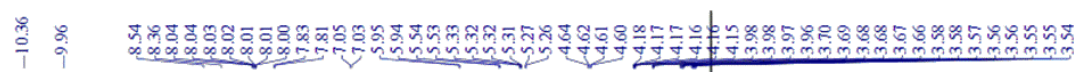

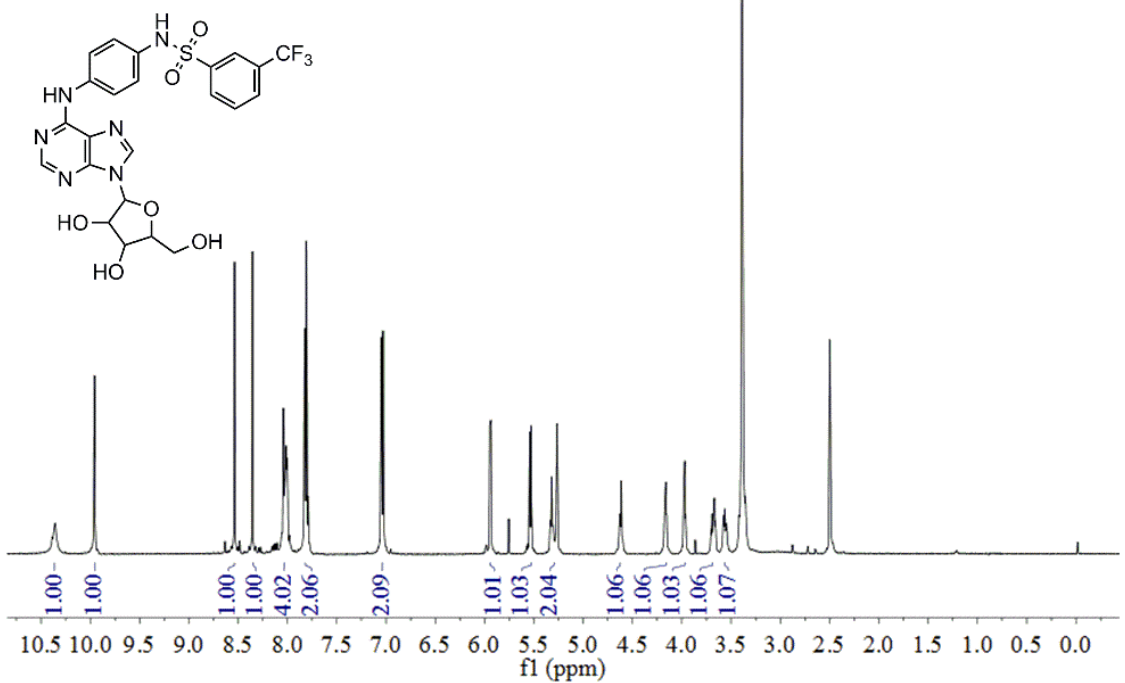

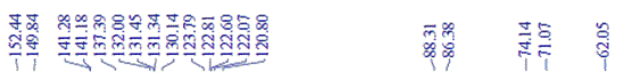
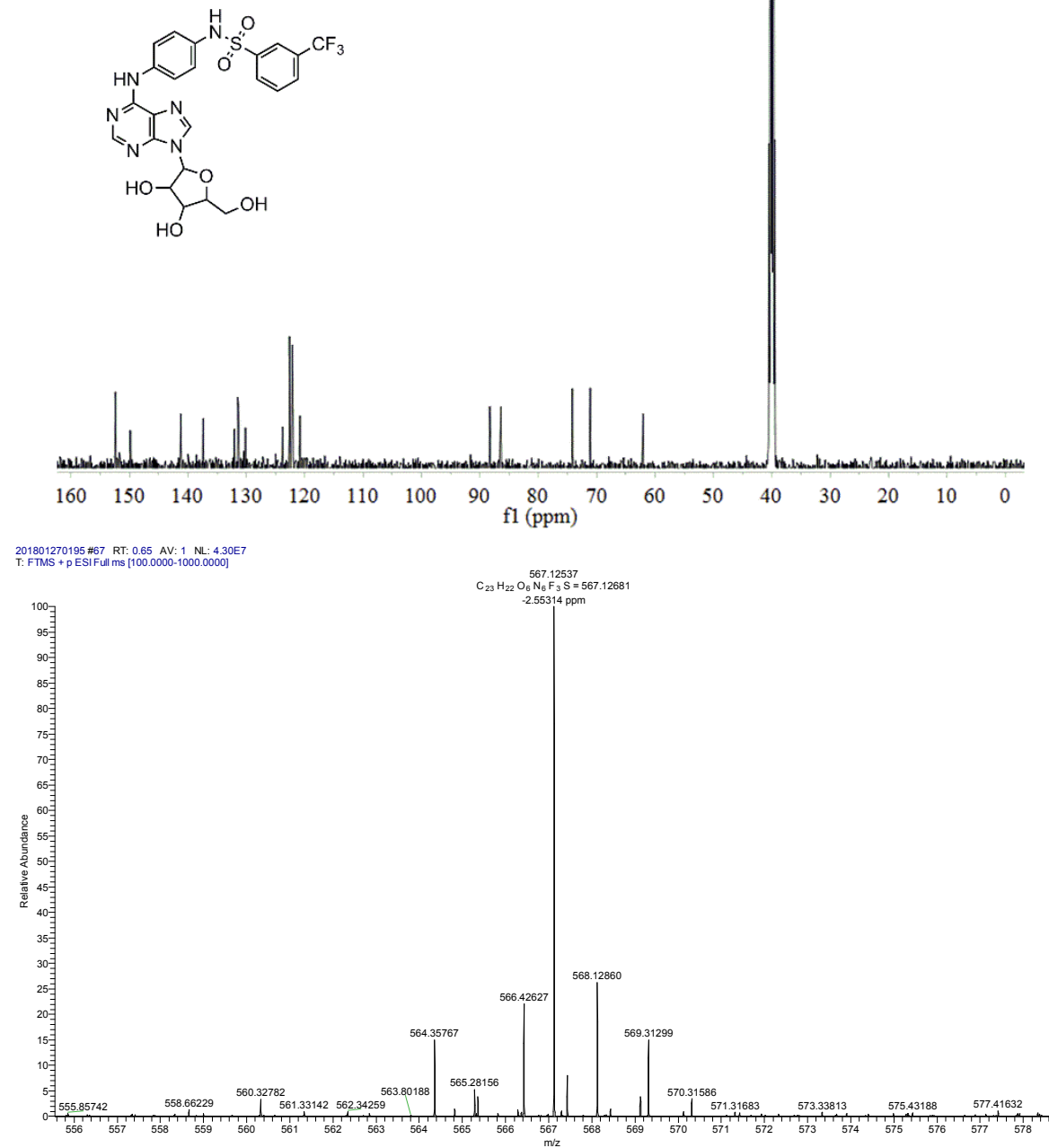

Spectral data for compound 19 


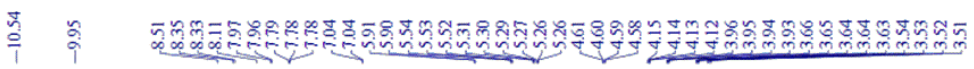

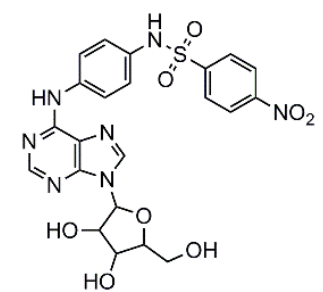

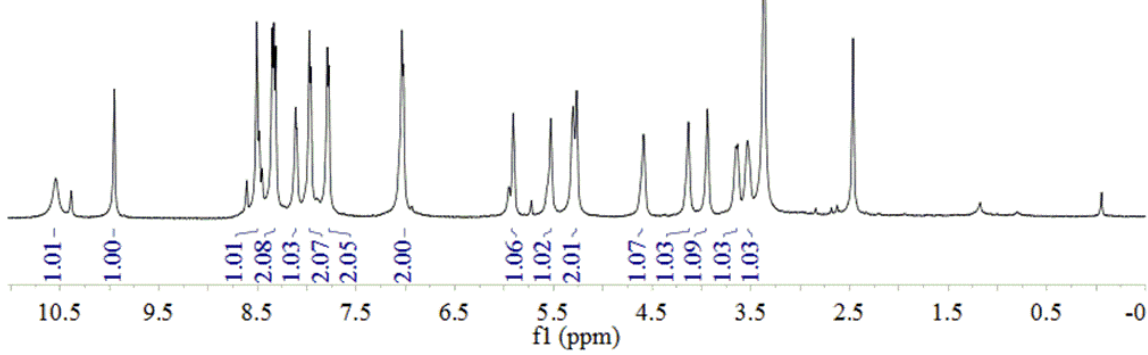

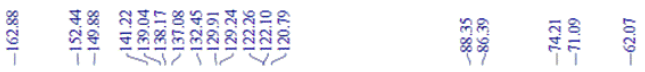
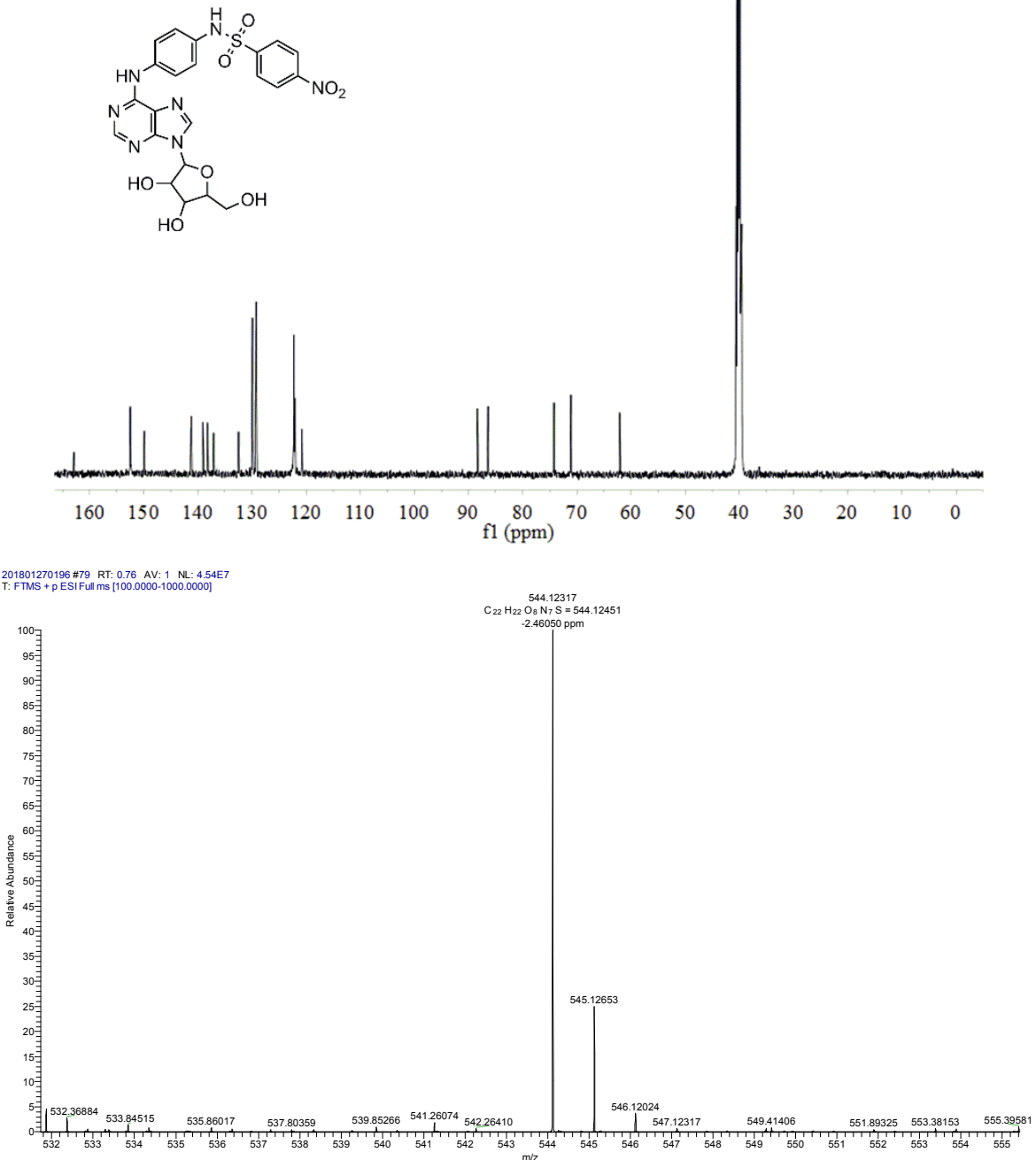

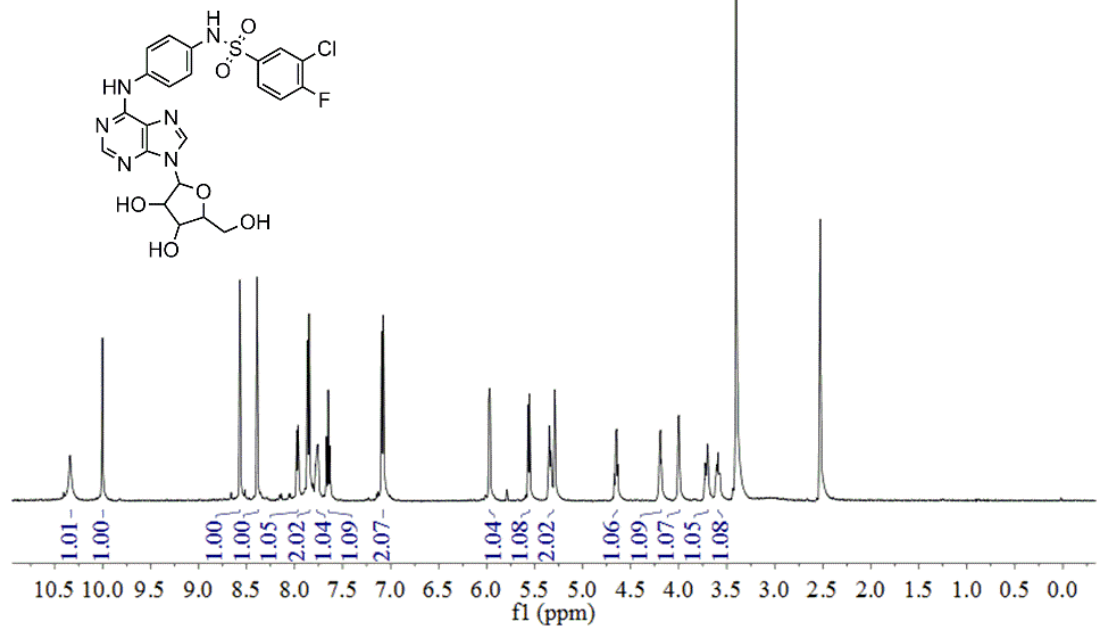

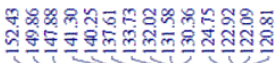

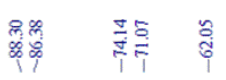
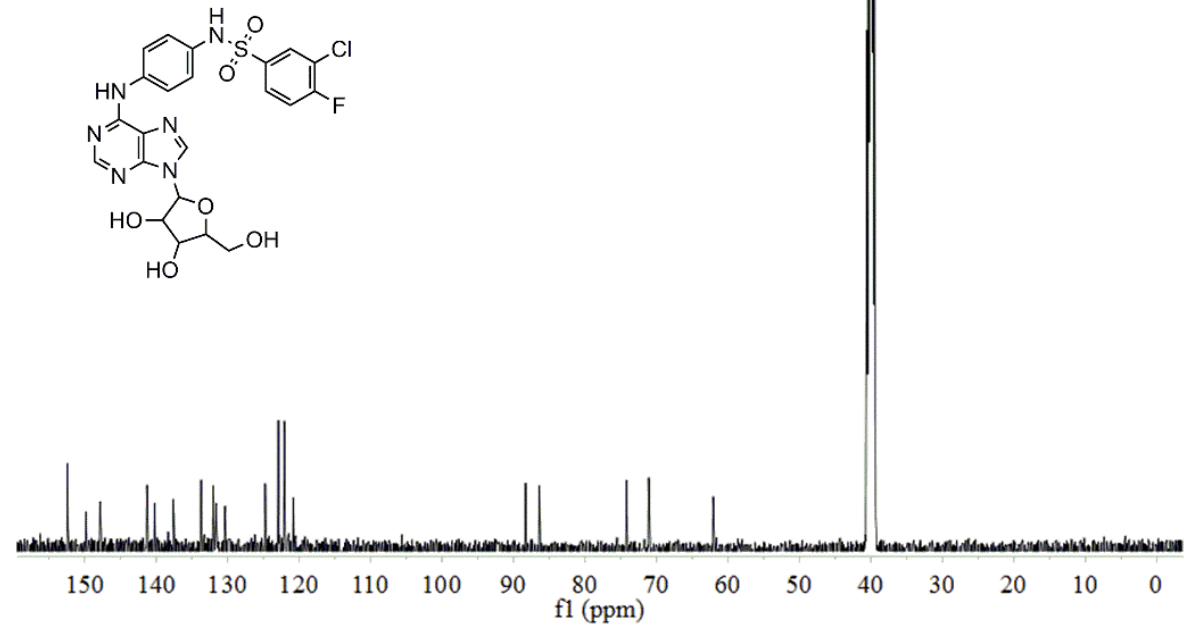

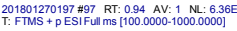

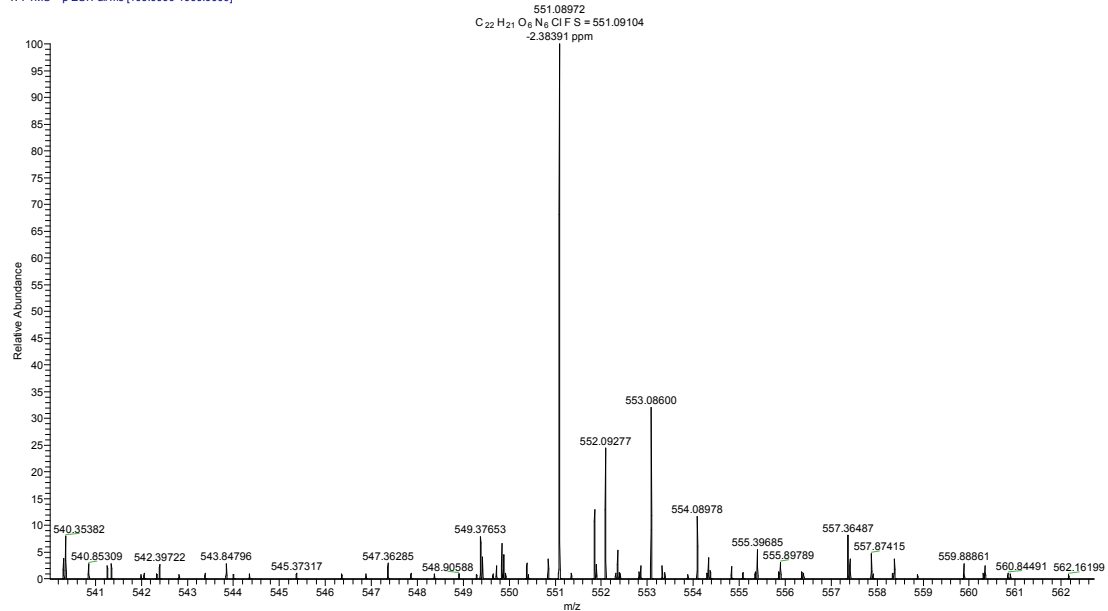

Spectral data for compound 21 

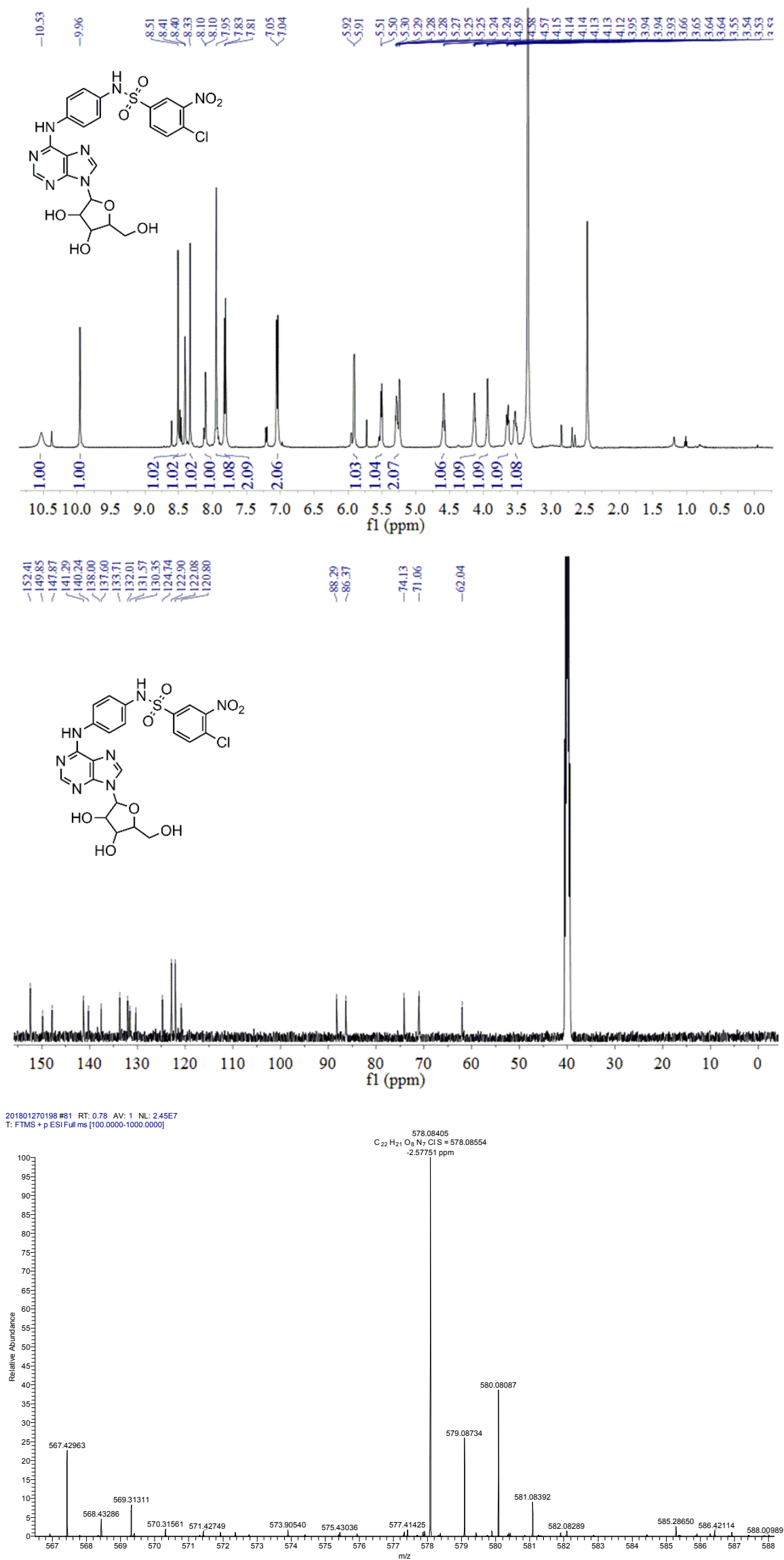

\section{Spectral data for compound 22}




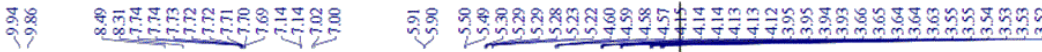
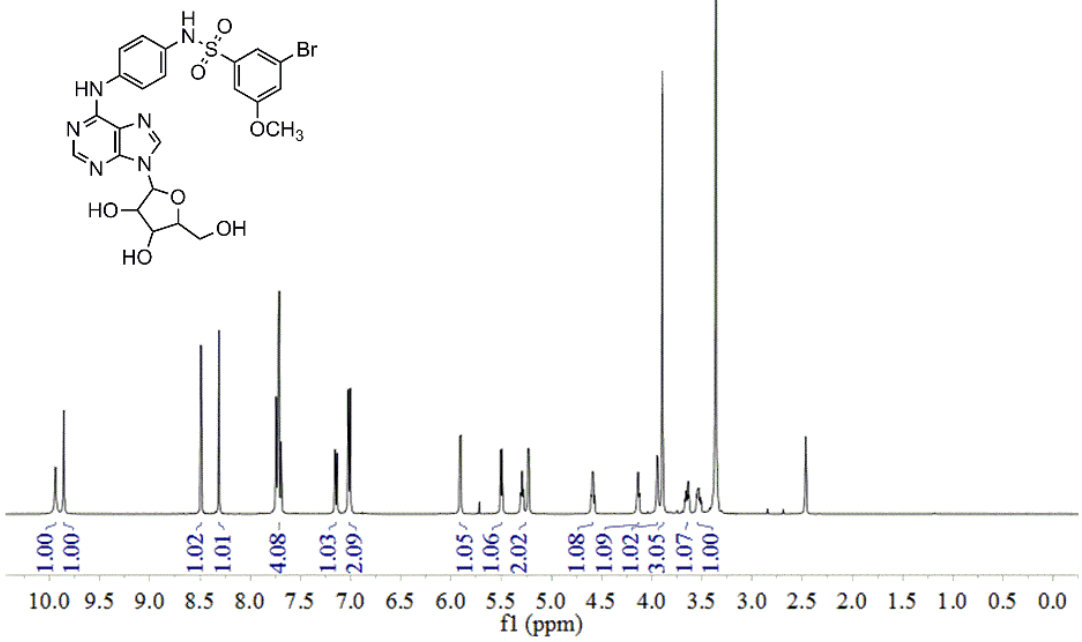

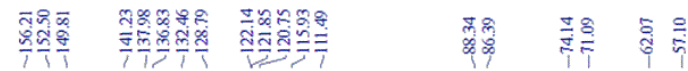

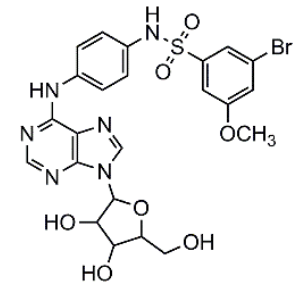
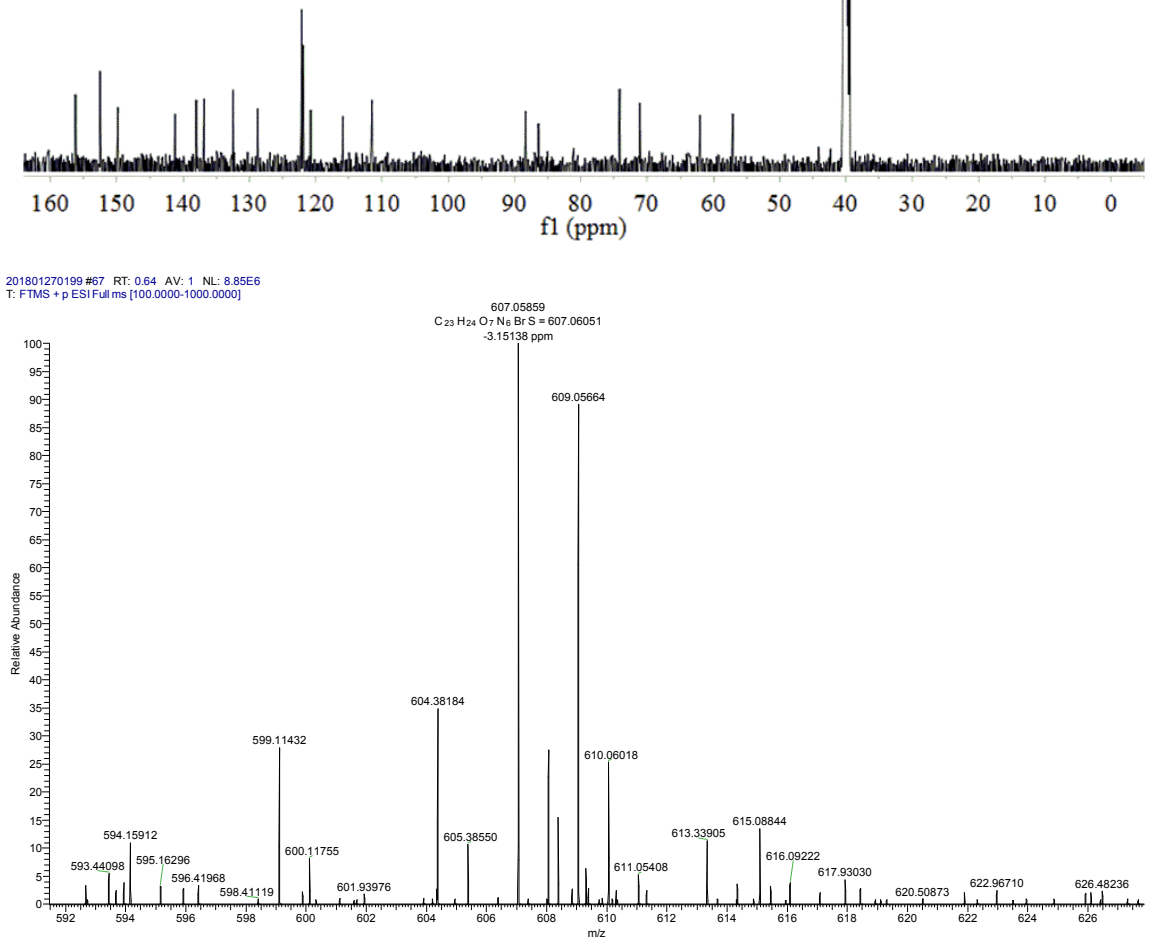

Spectral data for compound 23 


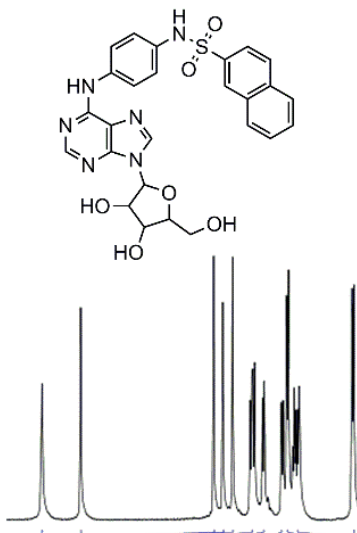

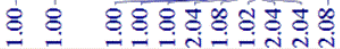

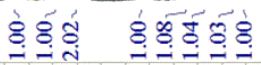

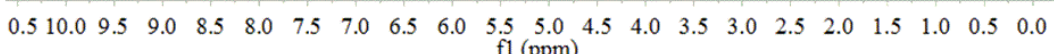

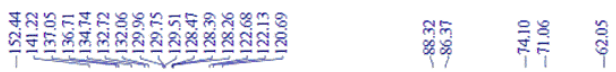<smiles>O=S(=O)(Nc1ccc(NC2=NN=CN3C2OC(n2cncn2)C3O)cc1)c1ccc2ccccc2c1</smiles>
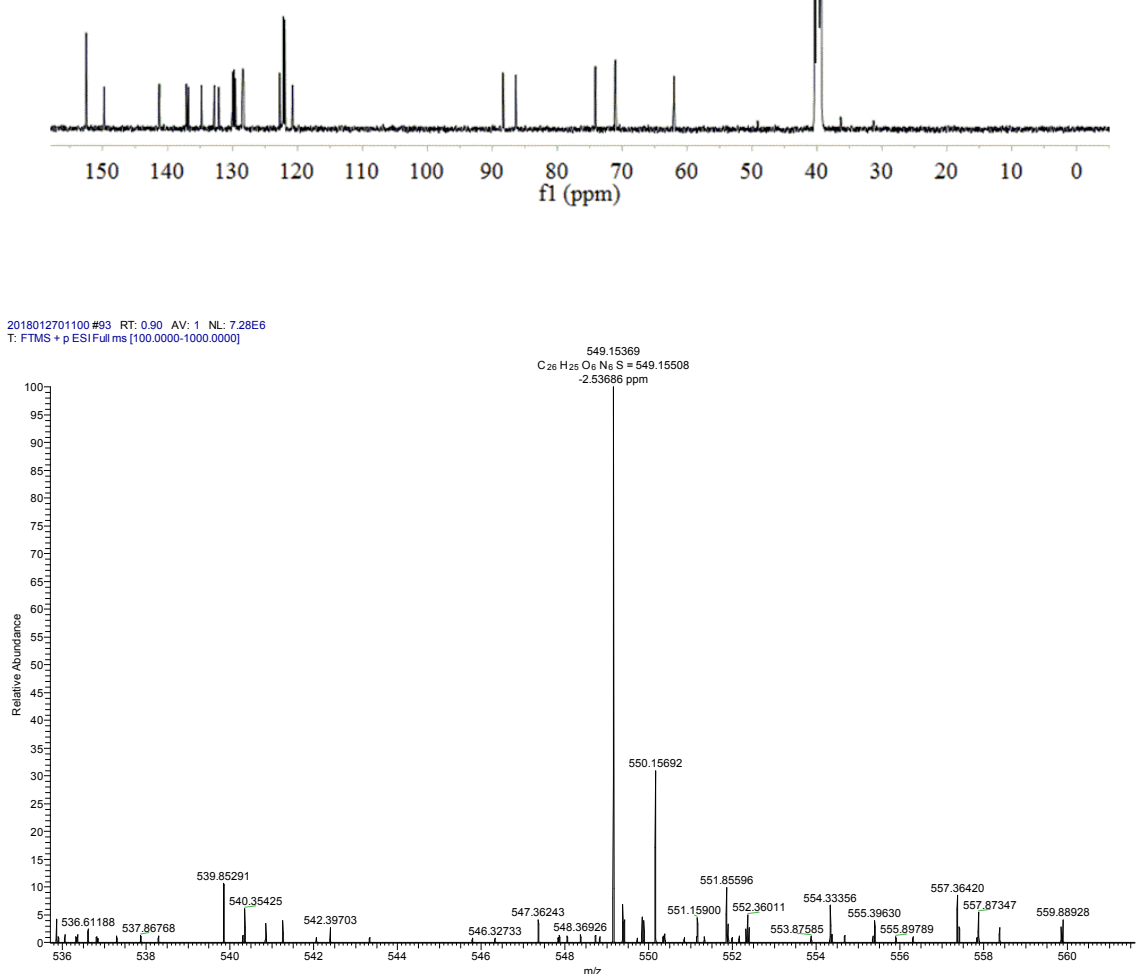

\section{Spectral data for compound 24}



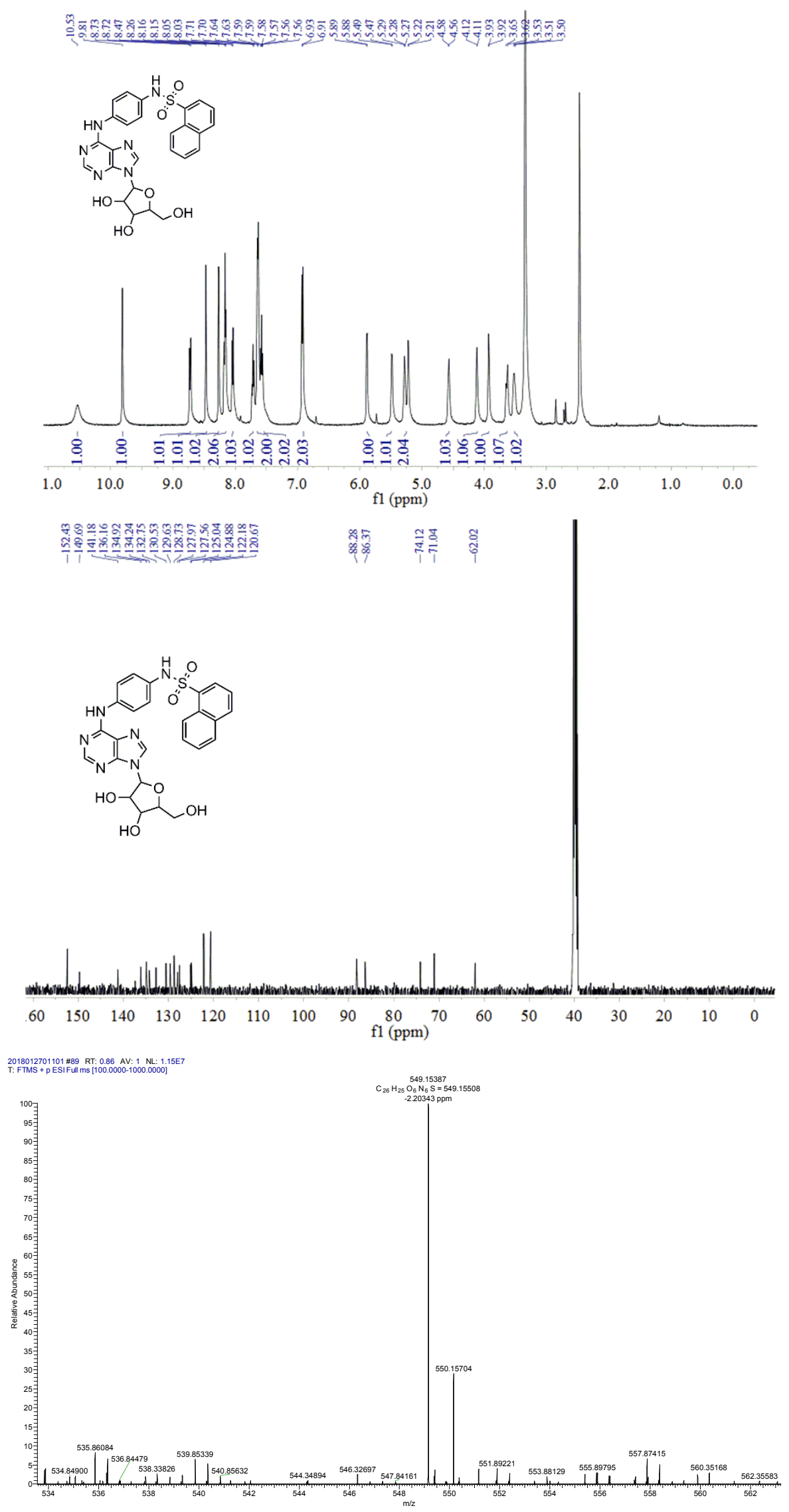

Spectral data for compound 25 


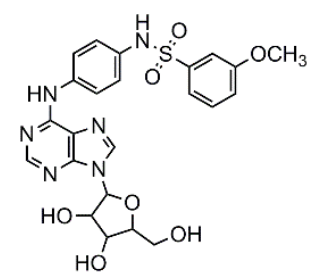
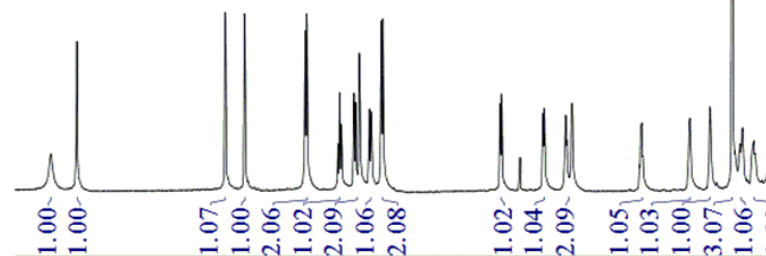

$\begin{array}{lllllllllllllllllllll}10.0 & 9.5 & 9.0 & 8.5 & 8.0 & 7.5 & 7.0 & 6.5 & 6.0 & 5.5 & 5.0 & 4.5 & 4.0 & 3.5 & 3.0 & 2.5 & 2.0 & 1.5 & 1.0 & 0.5 & 0.0\end{array}$

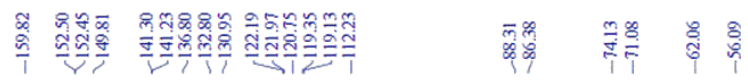

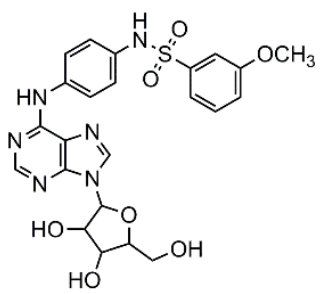
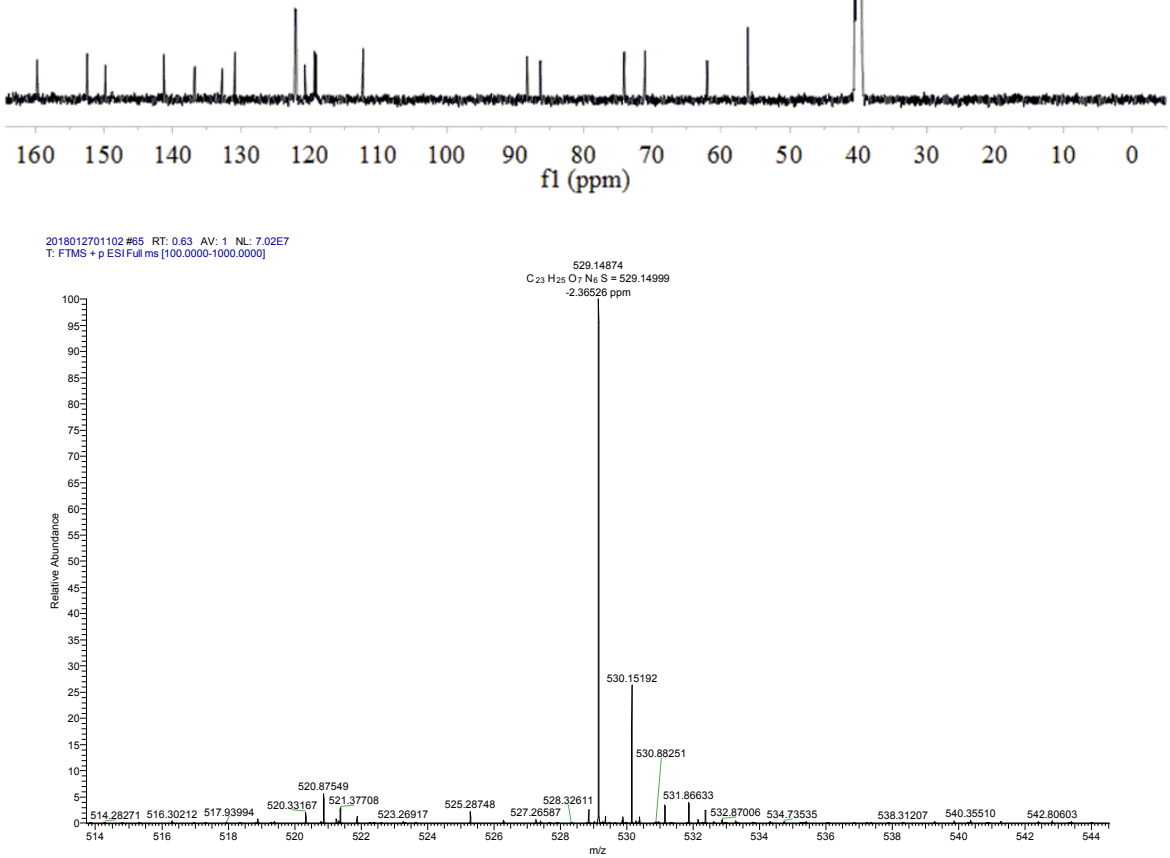